\title{
Modeling and simulation of tricycle landing gear at normal and abnormal conditions
}

Philip E. Evans

West Virginia University

Follow this and additional works at: https://researchrepository.wvu.edu/etd

\section{Recommended Citation}

Evans, Philip E., "Modeling and simulation of tricycle landing gear at normal and abnormal conditions" (2010). Graduate Theses, Dissertations, and Problem Reports. 2153.

https://researchrepository.wvu.edu/etd/2153

This Thesis is protected by copyright and/or related rights. It has been brought to you by the The Research Repository @WVU with permission from the rights-holder(s). You are free to use this Thesis in any way that is permitted by the copyright and related rights legislation that applies to your use. For other uses you must obtain permission from the rights-holder(s) directly, unless additional rights are indicated by a Creative Commons license in the record and/ or on the work itself. This Thesis has been accepted for inclusion in WVU Graduate Theses, Dissertations, and Problem Reports collection by an authorized administrator of The Research Repository @ WVU. For more information, please contact researchrepository@mail.wvu.edu. 


\title{
Modeling and Simulation of Tricycle Landing Gear at Normal and Abnormal Conditions
}

\author{
Philip E. Evans
}

Thesis submitted to the

\section{College of Engineering and Mineral Resources at West Virginia University}

\section{in partial fulfillment of the requirements}

for the degree of

\author{
Master of Science \\ in \\ Mechanical Engineering
}

\author{
Mario Perhinschi, Ph.D., Chair \\ Larry Banta, Ph.D. \\ Jacky Prucz, Ph.D.
}

Department of Mechanical and Aerospace Engineering

Morgantown, West Virginia 


\section{Abstract \\ Modeling and Simulation of Tricycle Landing Gear at Normal and Abnormal Conditions}

\section{Philip E. Evans}

This thesis presents the development of a simulation environment for the design and analysis of a tricycle landing gear at normal and abnormal conditions. The model is developed using superposition of the elastic and damping effects of each landing strut. The landing model is interfaced with an existing flight model based upon a tricycle landing gear system business jet aircraft within a Matlab/Simulink® simulation environment. The aircraft model is capable to portray several classes of landing failures, such as component degradation and jamming. The goals of this effort are oriented at creating tools for the design and analysis of fault tolerant control laws, landing gear development, and failure simulation in an academic setting. Formulation of the landing gear model at both normal and abnormal conditions is presented with detailed vector notation. Adjustments to alter the theoretical model to accurately portray real world limitations are also covered. Flowcharts of the GUIs used in implementing the failure simulator are included and discussed. The Simulink® program used to model the landing gear system at normal and abnormal conditions is described. Representative simulation results for each failure are included. The capability of simulating landing gear failures is shown to be plausible for the simulator. The adequate performance of the simulator is demonstrated and assessed qualitatively since experimental data was not available. This model serves as a starting point and general framework for the development of more accurate landing gear models for specific types of aircraft. 


\section{Table of Contents}

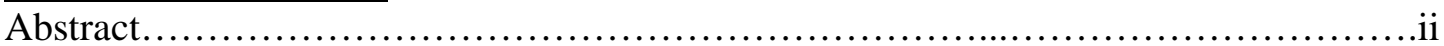

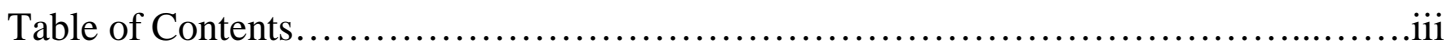

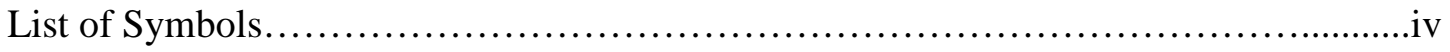

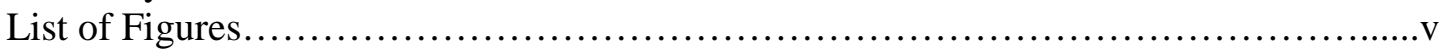

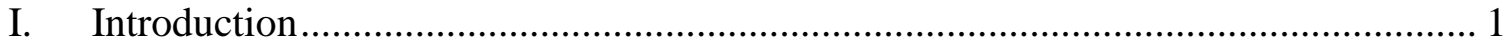

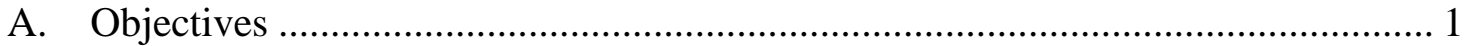

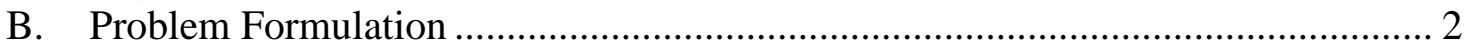

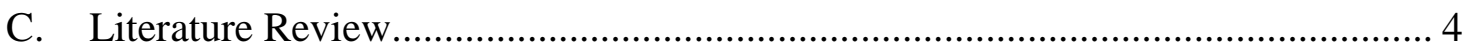

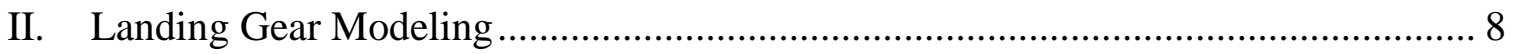

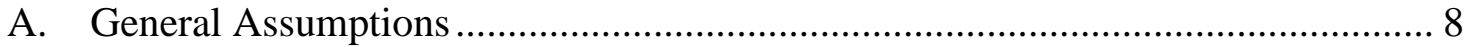

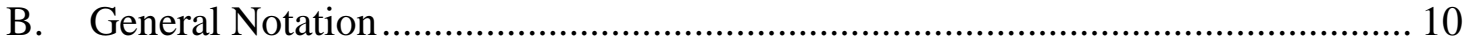

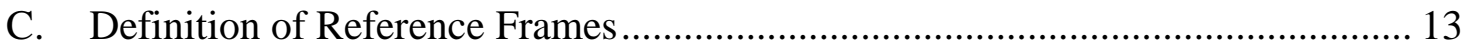

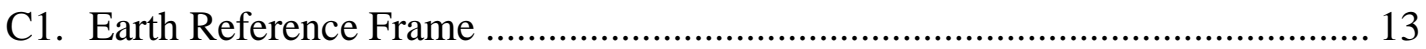

C2. Body Reference Frame …………........................................................... 14

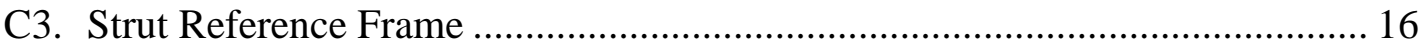

C4. Turning Reference Frame ……………………….................................... 17

D. Integration of the Aircraft Motion and Landing Gear Models ............................. 20

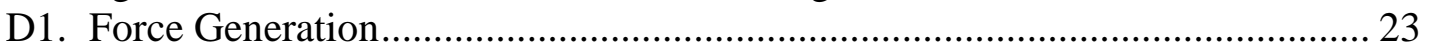

D2. Determining the Length of the Landing Gear............................................... 26

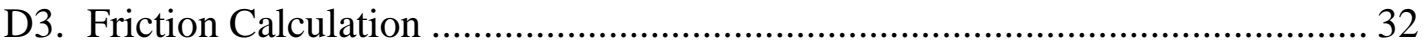

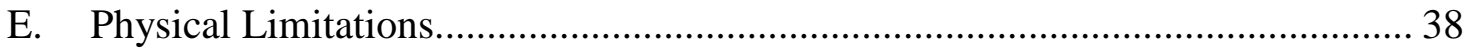

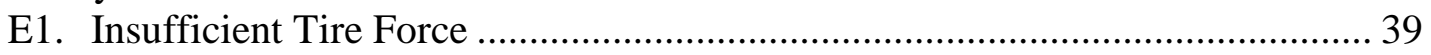

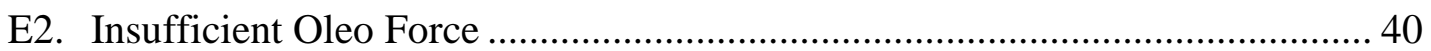

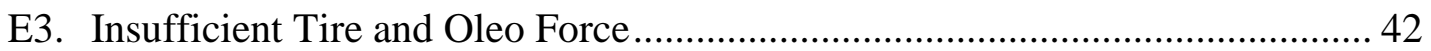

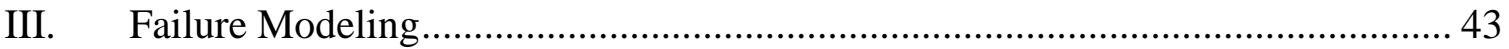

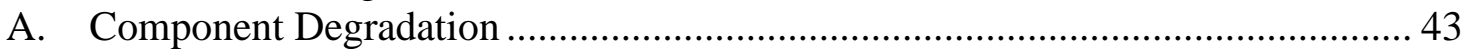

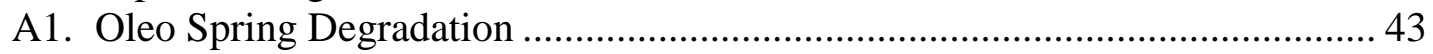

A2. Oleo Damper Degradation ............................................................................ 44

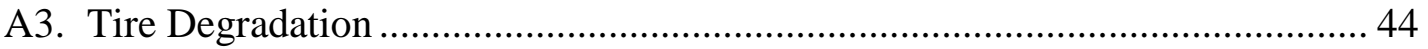

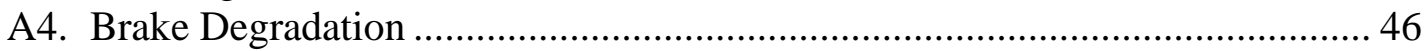

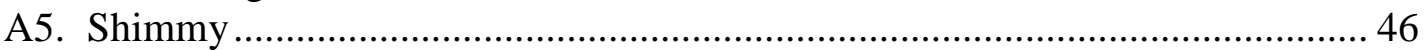

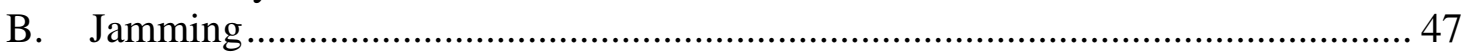

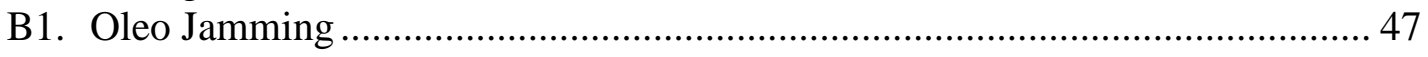

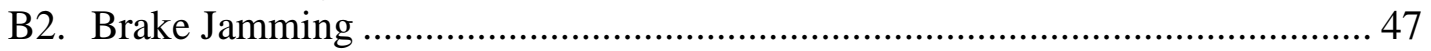

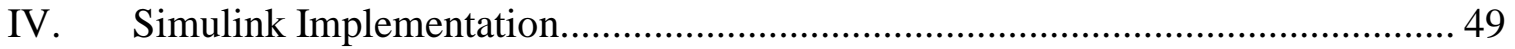

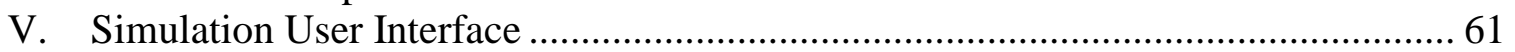

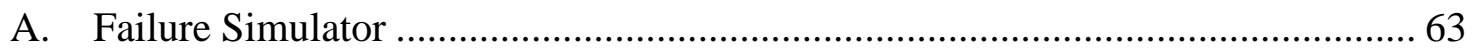

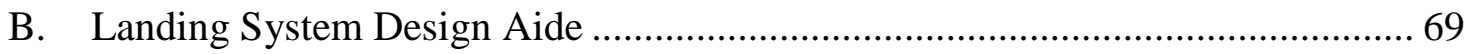

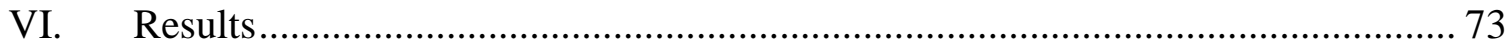

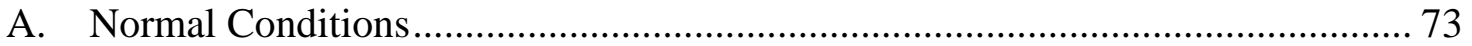

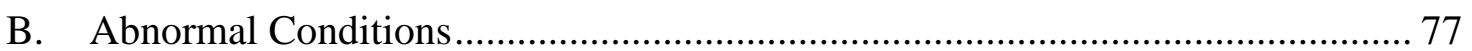

VII. Conclusions and Recommendations .................................................................... 97

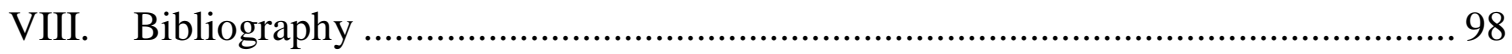

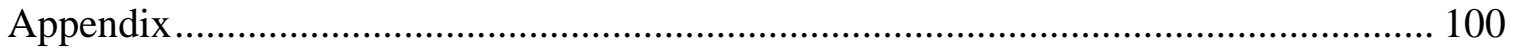

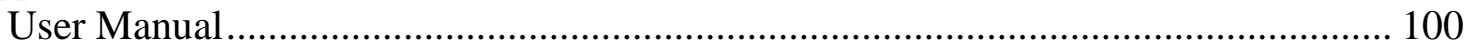




\section{List of Symbols}

\begin{tabular}{ll}
\multicolumn{1}{c|}{ Main Symbol } & \\
alt & Landing Surface Altitude \\
ARV & Arbitrary Real Variable \\
c & Damping Coefficient \\
CG & Center of Gravity \\
F & Force \\
g & Gravity \\
GCP & Ground Contact Point \\
I & Inertia \\
k & Spring Coefficient \\
L & Moment about the X-Axis \\
M & Moment about the Y-Axis \\
m & Mass of the Aircraft \\
N & Moment about the Z-Axis \\
P & Angular Velocity about the Body X-Axis \\
Q & Angular Velocity about the Body Y-Axis \\
R & Angular Velocity about the Body Z-Axis \\
Rad & Radius \\
RF & Reference Frame \\
SDL & Strut Deflection Line \\
t & Time \\
U & Body Reference Frame Longitudinal Velocity \\
V & Body Reference Frame Lateral Velocity \\
W & Body Reference Frame Vertical Velocity \\
X & X Component \\
Y & Y Component \\
Z & Z Component \\
&
\end{tabular}

\begin{tabular}{ll} 
Subscript & \\
\hline 1 & Oleo Component \\
2 & Tire Component \\
$\mathrm{a}$ & Point where the Fuselage Connects to the Strut \\
Aero & Aerodynamic Generated \\
$\mathrm{B}$ & Body Based \\
Balance & Variable Defined to Balance Forces and Moments \\
Brake & Braking Value \\
$\mathrm{E}$ & Earth Based \\
$\mathrm{f}$ & Failure Point \\
Failure & Failure Generated \\
$\mathrm{Friction}$ & Value Related to Friction \\
$\mathrm{g}$ & Point where the Strut is to Intersect the Ground \\
$\mathrm{L}$ & Left Wheel Related \\
$\mathrm{LG}$ & Landing Gear Generated
\end{tabular}




\begin{tabular}{ll} 
M & Main Wheel Related \\
Max & Maximum Value \\
Min & Minimum Value \\
$\mathrm{N}$ & Nose Wheel Related \\
Normal & Normal Response \\
O & Point of Origin \\
oleo & Oleo Component \\
orig & The Original Value \\
Pilot & Pilot Generated \\
$\mathrm{R}$ & Right Wheel Related \\
rot & Rotation Component \\
S & Strut Based \\
Sliding & Response Generated while Sliding \\
subscript & Generic Subscript Quantity \\
T & Turning Based \\
Threshold & Threshold Limit \\
Thrust & Thrust Generated \\
tire & Tire Component \\
Total & Sum of all Components in Same Direction \\
w & Reference Point of the Wheel \\
$\mathrm{x}$ & X Component \\
y & Y Component \\
z & Z Component \\
& \\
Greek Symbols & \\
\hline$\varphi$ & \\
$\theta$ & Roll Attitude Angle \\
$\psi$ & Pitch Attitude Angle \\
& Yaw Attitude Angle
\end{tabular}




\section{List of Figures}

Figure 1. Depiction of changing from the Earth RF to the body RF 16

Figure 2. Depiction of changing from the body RF to the strut RF 17

Figure 3. Depiction of changing from the strut RF to the turning RF 18

Figure 4. Depiction of the RF's on an aircraft body. 19

Figure 5. General diagram of a flight simulation algorithm 21

Figure 6. Dynamic model of a landing gear strut with the free body diagram 24

Figure 7. Points of interest dynamically applied to a linear strut 24

Figure 8. Depiction of geometric components used in determined strut length 24

Figure 9. Dynamic model of a landing gear with insufficient tire force 39

Figure 10. Dynamic model of a landing gear with insufficient oleo force 40

Figure 11. Dynamic model of a landing gear with insufficient tire and oleo force 42

Figure 12. General diagram of the different modules of the simulation environment 50

Figure 13. Highest level of landing gear modeling block. 51

Figure 14. View of the landing gear modeling stages. 52

Figure 15. Calculating the strut length. 53

Figure 16. View of different landing gear modes. 54

Figure 17. Normal dynamic model of landing gear forces.

Figure 18. Internal components of friction calculator. 56

Figure 19. Maximum friction and velocity calculation. 57

Figure 20. Routing the frictional forces for balanced motion. 58

Figure 21. Applying the frictional force algorithm. 58

Figure 22. Summing of the landing gear's moments. 59

Figure 23. Technique for using timed failure trigger. $\quad 60$

Figure 24. Technique for determining tire blowout. 60

Figure 25. First GUI in employing the simulator. 61

Figure 26. Flowchart of the entire simulator user interface. 62

Figure 27. Opting between predetermined and real time failure initiation. 63

$\begin{array}{ll}\text { Figure 28. Depiction of possible nose failures. } & 64\end{array}$

Figure 29. Selecting the location of the failure in the predetermined case. 64

Figure 30. Selecting the failure trigger. 66

$\begin{array}{ll}\text { Figure 31. Starting the aircrafts flight. } & 67\end{array}$

Figure 32. Control logic of the real time portion of the failure simulator. 68

Figure 33. GUI used to input design aide parameters.

Figure 34. Data entry techniques for designer tool. 71

Figure 35. Graph plotting logic for designer tool. $\quad 72$

Figure 36. Altitude when subjected to normal conditions. 73

Figure 37. Pitch attitude when subjected to normal conditions. $\quad 73$

Figure 38. Force generated by the nose when subjected to normal conditions. $\quad 74$

Figure 39. Force generated by the main when subjected to normal conditions. $\quad 74$

Figure 40. Distance along SDL to the hub when subjected to normal conditions. 74

Figure 41. Velocity under normal conditions with half thrust.

Figure 42. Altitude under normal conditions with half thrust. 75

Figure 43. Pitch attitude under normal conditions with half thrust. 75

Figure 44. Distance to the wheel hub under normal conditions with half thrust. 75 
Figure 45. Force generated by the nose under normal conditions with half thrust. 76

Figure 46. Available braking force with only 25\% brakes applied. 77

$\begin{array}{ll}\text { Figure 47. Altitude when subjected to a degraded strut spring. } & 78\end{array}$

Figure 48. Pitch angle when subjected to a degraded strut spring. 78

Figure 49. Distance along the SDL to the hub when subjected to a degraded strut spring.

Figure 50. Force generated by the nose when subjected to a degraded strut spring.

Figure 51. Force generated by the main when subjected to a degraded strut damper. 79

Figure 52. Pitch attitude when subjected to a degraded strut damper. 80

Figure 53. Roll attitude when subjected to a degraded strut damper. 80

Figure 54. Force generated by the nose when subjected to a jammed strut. $\quad 81$

Figure 55. Pitch attitude when subjected to a jammed strut. $\quad 81$

Figure 56. Distance along the SDL to the hub when subjected to a jammed strut. $\quad 81$

Figure 57. Altitude when subjected to a jammed strut. $\quad 81$

Figure 58. Velocity under half thrust with tire loss. 82

Figure 59. Pitch attitude under half thrust with tire loss. 82

Figure 60. Nose force under half thrust with tire loss. 82

Figure 61. Distance along SDL to wheel hub under half thrust with tire loss. $\quad 82$

Figure 62. Turning angle under half thrust with tire blowout. 83

Figure 63. Side force under half thrust with tire blowout. 83

Figure 64. Turning angle generated by shimmy effects. 84

Figure 65. Roll attitude generated by shimmy effects. 84

Figure 66. Pitch attitude when the nose gear is not deployed. 85

Figure 67. Altitude when the nose gear is not deployed. 85

Figure 68. Velocity when the nose gear is not deployed. 85

Figure 69. Nose force when the nose gear is not deployed. 85

Figure 70. Yaw produced with left brake degradation. 86

Figure 71. Available braking force with left brake degradation. 86

Figure 72. Altitude when subjected to a main oleo spring degradation. $\quad 88$

Figure 73. Pitch attitude when subjected to a main oleo spring degradation. $\quad 88$

Figure 74. Main strut force when subjected to a main oleo spring degradation. $\quad 88$

Figure 75. Main tire force when subjected to a main oleo spring degradation. $\quad 88$

Figure 76. Altitude when subjected to a main tire spring degradation. 89

Figure 77. Pitch attitude when subjected to a main tire spring degradation. 89

Figure 78. Main strut force when subjected to a main tire spring degradation. $\quad 89$

Figure 79. Main tire force when subjected to a main tire spring degradation. $\quad 89$

Figure 80. Altitude when subjected to a main oleo damper degradation. 90

Figure 81. Pitch attitude when subjected to a main oleo damper degradation. $\quad 90$

Figure 82. Main strut force when subjected to a main oleo damper degradation. $\quad 91$

Figure 83. Main tire force when subjected to a main oleo damper degradation. $\quad 91$

Figure 84. Nose strut force when subjected to a main oleo damper degradation. $\quad 91$

Figure 85. Nose tire force when subjected to a main oleo damper degradation. $\quad 91$

Figure 86. Altitude when subjected to a nose oleo spring degradation. 92

Figure 87. Pitch attitude when subjected to a nose oleo spring degradation. 92

Figure 88. Nose strut force when subjected to a nose oleo spring degradation. $\quad 92$

Figure 89. Nose tire force when subjected to a nose oleo spring degradation. 92 
Figure 90. Altitude when subjected to a nose tire spring degradation. 93

Figure 91. Pitch attitude when subjected to a nose tire spring degradation. 93

Figure 92. Nose strut force when subjected to a nose tire spring degradation. 94

Figure 93. Nose tire force when subjected to a nose tire spring degradation. 94

Figure 94. Altitude when subjected to a nose oleo damper degradation. 95

Figure 95. Pitch attitude when subjected to a nose oleo damper degradation. 95

Figure 96. Nose strut force when subjected to a nose oleo damper degradation. 95

Figure 97. Nose tire force when subjected to a nose oleo damper degradation. 95

Figure 98. Main strut force when subjected to a nose oleo damper degradation. 96

Figure 99. Main tire force when subjected to a nose oleo damper degradation. 96 


\section{Introduction}

\section{A. Objectives}

There are many flight simulators in existence. These range from extremely basic, (as seen with some low-grade video games), to the extremely detailed, (pilot trainers for the armed services). The more advanced simulations, the ones used for training, have additional failure features that can be inserted into the model so that the pilot can experience a multitude of malfunctions. These failure features are programmed to occur while the aircraft is in flight or basic considerations while on the ground. What if more than the common failures were to happen while on the ground? How will the pilot be prepared for this scenario?

With these questions in mind, the following goals must be met to arrive at the desired solution to the presented problems. A model of the tricycle landing gear needs to be developed. This model must be able to function at both normal and abnormal operating conditions. After the model is created, it needs to be implemented in conjunction with a working flight model. To ensure that the representation of the landing gear model is accurately constructed, the combination of the two models should be rigorously tested to make sure that all of the failures relate to real-world situations. Also, all of the failures should be tested to ensure that none of them cause terminal errors in the working flight model.

Pilots are not the only beneficiaries of such a simulation environment. The engineers who design the landing gear system also are affected by failures and abnormal flight conditions. Since aircraft dynamic qualities will degrade from the parameters it 
was designed to have because of weather and usage influences, landing gear designers should have the ability to test for an acceptable variance. This variance is generated by real world applications having different weather conditions, landing surfaces, and aircraft maintenance, among other aspects. If a large enough variance still allows for safe aircraft landing, the design is sound. With this in mind, the simulator should have additional capabilities, which can be used for this purpose.

The simulator can also be used in an academic environment. This would allow for students to investigate how altering dynamic characteristics such as spring factors and damping would affect the response of the system. Additionally, the students would be able to experience abnormal conditions. Through this, they would be able to gain first hand knowledge of how this engineering system can act when components fail.

\section{B. Problem Formulation}

The set of flight simulation tools developed at West Virginia University for academic and research purposes is based on models implemented in Matlab® and Simulink®, which provide maximum modeling expansion capabilities, flexibility, and portability. A business jet model, including aerodynamic, propulsion, and general equations of motion, interfaced with the WVU 6 degrees-of-freedom (DOF) motionbased flight simulator served as a starting point for the landing gear modeling process. As will be discussed later in the thesis, the only way to make adjustments to the business jet model is to induce external forces and moments computed along the body axes onto the model that would simulate the landing gear when in contact with the landing surface. These forces and moments will be combined with the business jet's forces and moments 
and used in calculating its dynamic equations. Due to technical constraints, the model of the landing gear needs to be as computationally simple as possible, yet still capture the dynamic characteristics at both normal and abnormal conditions. These forces, and the moments that they create, should be generated by using superposition of elastic and damping effects for each landing gear strut. This is done by determining the force each component of the landing gear would produce and combining them.

Once an accurate algorithm for finding the force of the landing gear is created, the model must meet a few additional criteria to fulfill the objectives as previously stated. Most importantly, it must be able to land on any surface as long as a governing algorithm can be created for that surface. To accomplish this, geometric equations need to be applied to find the distance between a point and a surface along a given line. This technique will determine if the aircraft is in contact with the ground. Since this equation will be different for each landing surface, the most simplistic type will be used, landing on a horizontal smooth plane. This algorithm needs to be applied as both a failure simulator and a designer's aide. The designer's aide will operate identically to the flight simulator but with the inputs constrained and the geometric parameters of the aircraft available for the user to alter.

For a simulator to be used to train against failures, it must have a plethora of possible abnormalities that the model can portray. First, it must be able to handle an alteration to the dynamics of the oleo affecting either the spring or damper components. An oleo is a shock absorber that uses both oil and gas for its compression fluid ${ }^{[1]}$. The changes in the tire dynamic should also be modeled. Changing the spring constant is one way to do this. Another way is to remove the tire component completely; this can be 
done to simulate the loss of the tire, completely flat tire, or the unfortunate event of a blowout. Brakes are another part of the aircraft that differs over time and needs to be included on the list of possible failures. Shimmy is another type of abnormal condition considered in this thesis. It occurs when the torsion of the steering wheel undergoes an oscillating phenomenon. Lastly, parts of the landing gear system can become jammed, namely the oleo and the brakes. These abnormalities must all be able to be simulated to properly train the pilot against aircraft failures.

\section{Literature Review}

The first recorded flight simulator was created in 1909 to train pilots how to fly the Antoinette monoplane ${ }^{[2]}$. This simplistic device used two wheels for the pilot controls. Movement of the simulator was done by two outside assistants. The first flight simulator not based on an outside human force was the Link Trainer ${ }^{[3]}$. This device used pneumatics to give accurate instrument readings. Digital computers were not implemented into flight simulation until the 1960s. Through the use of computational devices, it has become possible not only to increase the accuracy of the flight simulation experience at normal conditions but also to inject sub-system failures into the flight model and to simulate a variety of abnormal conditions.

In general, simulator builders give limited attention to the accurate simulation of vehicle/ground interaction under abnormal conditions. Ground failures need to be modeled to give pilots some foreknowledge prior to being submitted to the abnormal conditions. Poor pilot inspections can miss small fractures that can cause a landing strut to collapse ${ }^{[4]}$. Robert Boser of AirlineSafety.com reports several aircraft having to land 
with their nose wheels stuck at a full ninety degree deflection ${ }^{[5]}$. Turning of the nose wheel is not the only failure it is commonly subjected to. Aircraft have belly landed when trying to touch down ${ }^{[6]}$. Niebuhr reports that this occurrence could be attributed to short circuiting in the electronics ${ }^{[7]}$. It is possible for the systems to return a false positive that the landing gear is deployed. In fact, over a five year period a hundred failures have been reported to the Federal Aviation Administration, FAA $^{[8]}$.

Modeling of a landing gear system seems to traditionally be a minor concern of aerospace engineers. For an aircraft to pass FAA compliance for Level 6 flight simulators, only acceleration and deceleration of the landing gear simulator system need to correspond with the physical aircraft ${ }^{[9]}$. In defining the landing gear system, Clark only approximates the landing gear as to being perpendicular to the body of the aircraft $^{[10]}$, for this work the landing gear will be able to deflect in any linear manner specified. By using a series of vector transformations, the model can be made more accurate without any deterring effects on the simulation.

There have been some major advancements made on modeling of landing gear, however. A precise tire model has been derived ${ }^{[11]}$. Unfortunately, the level of complexity would add undue strain to an already computationally struggling model. Braking effects are another topic that has been researched ${ }^{[12]}$. Again, this is beyond the model's complexity threshold. To aid in making landings safer, a literature survey was performed on the vibrations an aircraft undergoes upon being in contact with a landing surface $^{[13]}$.

Active and semi-active control systems are some strategies under review to help eliminate forces and vibrations transmitted through contact with the landing surface. 
Ghiringhelli looked into testing of semiactive landing gear for general aviation aircrafts because it's simpler, lighter, and safer than active control ${ }^{[14]}$. The Air Force looked into active control to limit vibration fatigue of aircraft components ${ }^{[15]}$. An overview of optimum control strategies and possible future advancements is given by Mikułowski and Jankowski ${ }^{[16]}$.

Shimmy reduction is another major area of development that has received lots of interest to minimize the effect of vibration on the taxiing of the aircraft. Shimmy is an oscillatory rotation of the steering wheel of the aircraft generated by self-excitation. There are several techniques under development to eliminate and reduce this phenomenon. A closed-loop shimmy damper has been examined using root locus plots for use with a hydraulic steering system based on Moreland's point contact model ${ }^{[17]}$. By using another closed-loop feedback control scheme based on the formulation as presented by $\mathrm{Li}$, an active torsional magneto-rheological fluid-based damper has also been tested $^{[18]}$. Predictive control is another type of anti-shimmy device for the model set out by Somieski ${ }^{[19]}$. A closed-form analytical solution for shimmy has been found for a simplified linear nose-wheel landing gear model ${ }^{[20]}$.

Creating a landing simulator is not a new endeavor. Modelica-Dymola, a commercial modeling environment based on the Modelica language, has been proven to be capable of meeting these parameters and modeling a landing gear system ${ }^{[21]}$. To utilize this package, however, an entire new flight model would need to be created along with gaining an additional software package. Recently, Simulink has added a toolbox set with the capabilities of modeling the components used in a landing gear system ${ }^{[22]}$. This system is unable to be used since it creates difficulties in implementing component 
failures. The model must be created from scratch to allow for the failures to be applied. The equations used in this work are derived using Kane's Dynamics: Theory and Applications naming techniques as a pattern ${ }^{[23]}$. Dreier's work on landing gear modeling from Introduction to Helicopter and Tiltrotor Flight Simulation ${ }^{[24]}$ serves as a basis for the model.

The main reason that this research is being completed is to initialize the creation of a modeling technique that would generate the abnormalities created when parts of the landing gear sub-system fails. Once this is done, pilots will be able to become familiarized with the unfortunate event of parts of the landing gear failing during landing, taxiing, or takeoff. Once this familiarization is gained, they will know how to react to the failed situations. With the proper trained reaction, the pilot may be able to salvage the failure situation, saving the rest of the aircraft, but more importantly, the lives of the people on the aircraft. To accomplish this goal, the model will use the accepted dynamic model of a landing strut, namely the oleo modeled as a spring and damper system and the tire as only a spring. This classic model will be applied in a new aspect with the ability for the struts to not necessarily be perpendicular to the landing surface on touchdown. With non-perpendicular landing gear, the struts are allowed to rotate with the aircraft. A new technique to find the distance from the aircraft to the ground is needed to compensate for non-perpendicular struts. Additionally, being able to test changes in geometric and dynamic components of the landing gear to view the change in the response of the aircraft dynamics is also a novel idea. To test the creation of this model, the landing gear will be operated in conjunction with a model developed by other students at West Virginia University. 


\section{Landing Gear Modeling}

\section{A. General Assumptions}

In order to model the dynamics of the landing gear system, a few assumptions and simplifications have been made. These assumptions are of three different types. Limiting assumptions are those that constrain the landing gear model to be suitable for only standard aircraft but can be later expanded for the use in modeling different configurations. Examples of these are using the common tricycle landing gear layout and that the landing gear components along with the control surfaces of the aircraft are the only non-rigid parts of the aircraft. Computational assumptions are made so that the simulation speed is not an issue in the modeling of the landing system. Lastly, simplifying assumptions are used to lower the complexity of the model. A more rigorously precise model can forgo these assumptions for better results. These types of assumptions are that the dynamic coefficients are constant, ground induced aerodynamic phenomena are ignored (such as downdraft), the landing gear deflects linearly, and the landing surface is stationary and horizontally flat.

The limiting assumptions simplify the model so that a rigid sound model for standard aircraft landings can be made. Considering only tricycle landing gear allows for a majority of different aircraft to be employed since it is the most common configuration. Adjusting the model to be able to handle more than three landing struts can be done with additional customization. Modeling a non-rigid aircraft body would greatly increase the level of complexity without adding any real gain to the system. If an aircraft is unable to bend, twist, or break asunder, several calculations can be ignored. 
The computational assumption is made to deal with the simulation speed. For a pilot training device to be useful, it must run in real time. To operate in real time, there is a limitation to the number of calculations that can be completed per second. If this number is too low, the results from the landing gear dynamics could be incorrect due to inadequate integration step size. The Navy found the running speed of the landing system calculations to be lacking at sixty hertz since an aircraft is capable of descending at such high velocities ${ }^{[25]}$. Through testing, it was found that the model needed to run at a thousand hertz to be properly integrated with some of the possible damping and spring coefficients. As history has shown, the maximum number of calculations a computer can make per second will increase, making this issue a moot point. To make this issue less of an impact until more advanced computers are created, when there are multiple techniques to derive a solution the model should be created to be as computationally efficient as possible.

Several parameters are considered constant, which greatly lowers the complexity of the modeling environment. These constant parameters are the spring coefficient, damper coefficient, and gravity. Incorporating these as dynamic values requires the usage of look-up tables. To properly employ look-up tables the entirety of the deflection range must be mapped. Since different aircraft have different deflection ranges, using an approximation of these values allows for the simulator to cater to a wider range of aircrafts.

Ground induced aerodynamic phenomena are ignored because they are too complex. For an example, downdraft will be discussed. Downdraft occurs when an aircraft tries to takeoff too soon after another aircraft. The degree of effect is dependent 
on the aircraft size and geometry, time, and weather conditions, to name a few factors. If these types of effects are to be experienced, they can be manually inserted by the pilot's instructor, directly into the aerodynamic model.

Most landing gear struts do not deflect in a linear manner. Two great examples are when the tire deflection and oleo deflection are not linear or when the landing gear system decompresses in an arc. By adding the instantaneous force vectors generated by the tire and the oleo, new strut deflection lines (SDLs) are created. These instantaneous SDLs can then be used with a look-up table so that the correct orientation can be employed. If the differences in the SDLs are relatively small, then they can be approximated by one mean SDL. This approximation is used in this project.

Lastly, a horizontally planar and steady landing surface is used with this project. As will later be discussed, one of the largest determinations in the modeling of the landing gear reactions is finding the distance from the aircraft to the landing surface along the SDL. If an algorithm can be developed to define the equation for a dynamic complex surface, this new system can be engaged instead. That said, finding the algorithm could be quite difficult and only useful to a certain landing surface under certain conditions, such as training pilots for landing on an aircraft carrier at sea.

\section{B. General Notation}

In defining the landing gear system, several geometric entities must be used. A short description of how each of these devices is named and notated follows below.

The most basic component of geometry that needs to be properly introduced is the reference frame. Each reference frame, $(\mathrm{RF})$, is created so as to coincide with a rigid 
structure as previously set forth as an assumption. The shorthand notation for each reference frame is given as $\mathrm{RF}_{\text {subscript }}$. The subscript correlates to the specific reference frame, such as E for the Earth reference frame. Following this naming technique, $\mathrm{O}_{\text {subscript }}$ stands for the point of origin of a coordinate system associated to that specific reference frame. The corresponding axes are named in a similar manner with a vector between two points, namely the origin and a point on that vector such as $\mathrm{X}_{\text {subscript. }} \mathrm{A}$ more detailed description of how the parts of the reference frames correlate to the reallife application follows shortly.

Points are labeled by using a capital letter. The position vector of point B with respect to point $\mathrm{A}$ is denoted as:

$\vec{r}^{A B}=\overrightarrow{A B}$

The velocity vector of point A with respect to $R F_{\gamma}$ is by definition the time derivative of the position vector of A with respect to $O_{\gamma}$ taken with respect to $R F_{\gamma}$. It is denoted as:

$$
{ }^{\gamma} \vec{v}^{A}=\frac{{ }^{\gamma} d\left(\vec{r}^{O_{\gamma} A}\right)}{d t}=\frac{{ }^{\gamma} d\left(\overrightarrow{O_{\gamma} A}\right)}{d t}
$$

In general, the time derivative of a vector $\vec{V}$ with respect to reference frame $R F_{\gamma}$ is denoted by:

$$
\frac{\gamma d \vec{V}}{d t}=(\dot{\vec{V}})_{\gamma}
$$

The rotation vector of a reference frame $R F_{\gamma}$ or the rigid body associated to it, with respect to a reference frame $R F_{\delta}$ is denoted by ${ }^{\delta} \vec{\omega}^{\gamma}$. 
The time derivative of a vector $\vec{V}$ with respect to reference frame $R F_{\gamma}$ can be expressed in terms of the derivative of the same vector with respect to $R F_{\delta}$ according to the relationship:

$\frac{{ }^{\gamma} d \vec{V}}{d t}=\frac{\delta}{d t} d \vec{V}+{ }^{\gamma} \vec{\omega}^{\delta} \times \vec{V} \quad$ or $\quad(\dot{\vec{V}})_{\gamma}=(\dot{\vec{V}})_{\delta}+{ }^{\gamma} \vec{\omega}^{\delta} \times \vec{V}$

Often, a vector $\bar{V}$ needs to be expressed in terms of its components with respect to a system of coordinates associated to (or simply said, with respect to) a reference frame $R F_{O}$ as:

$[\bar{V}]_{O}=\left[\begin{array}{c}V_{X} \\ V_{Y} \\ V_{Z}\end{array}\right]_{O}$

The magnitude $V$ of vector $\bar{V}$ is given, of course, by:

$V=|\bar{V}|=\sqrt{V_{X}^{2}+V_{Y}^{2}+V_{Z}^{2}}$

Let the components with respect to $R F_{O}$ of two vectors $\bar{V}_{1}$ and $\bar{V}_{2}$ be respectively: $\left[\bar{V}_{1}\right]_{O}=\left[\begin{array}{lll}V_{1 X} & V_{1 Y} & V_{1 Z}\end{array}\right]^{T} o,\left[\bar{V}_{2}\right]_{O}=\left[\begin{array}{lll}V_{2 X} & V_{2 Y} & V_{2 Z}\end{array}\right]^{T} o$

The cross product of vectors $\bar{V}_{1}$ and $\bar{V}_{2}$ can then be expressed in components as:

$\left[\bar{V}_{1} \times \bar{V}_{2}\right]_{O}=\left[\begin{array}{ccc}0 & -V_{1 Z} & V_{1 Y} \\ V_{1 Z} & 0 & -V_{1 X} \\ -V_{1 Y} & V_{1 X} & 0\end{array}\right]_{O} \cdot\left[\bar{V}_{2}\right]_{O}$

The $3 \times 3$ matrix can be defined as the components of a tensor, the skew-symmetric tensor $\underline{\tilde{\mathrm{V}}_{1}}$, in other words: $\left[\tilde{\mathrm{V}}_{1}\right]_{\mathrm{A}}=\left[\begin{array}{ccc}0 & -\mathrm{V}_{1 \mathrm{z}} & \mathrm{V}_{1 \mathrm{y}} \\ \mathrm{V}_{1 \mathrm{z}} & 0 & -\mathrm{V}_{1 \mathrm{x}} \\ -\mathrm{V}_{1 \mathrm{y}} & \mathrm{V}_{1 \mathrm{x}} & 0\end{array}\right]_{\mathrm{A}}$. This allows us to write the cross product with tensor notation: 


$$
\overline{\mathrm{V}}_{1} \times \overline{\mathrm{V}}_{2}=\underline{\tilde{\mathrm{V}}_{1}} \overline{\mathrm{V}}_{2}
$$

The components of a vector $\bar{V}$ with respect to $R F_{A}$ can be obtained by pre-multiplying the components of the same vector with respect to $R F_{B}$ by the $3 \mathrm{X} 3$ transformation matrix $L_{A B}:$

$$
[\bar{V}]_{A}=L_{A B}[\bar{V}]_{B}
$$

where $L_{A B}$ depends on the Euler angles (see next section):

$$
L_{A B}=\left[\begin{array}{ccc}
\cos \psi & -\sin \psi & 0 \\
\sin \psi & \cos \psi & 0 \\
0 & 0 & 1
\end{array}\right]\left[\begin{array}{ccc}
\cos \theta & 0 & \sin \theta \\
0 & 1 & 0 \\
-\sin \theta & 0 & \cos \theta
\end{array}\right]\left[\begin{array}{ccc}
1 & 0 & 0 \\
0 & \cos \phi & -\sin \phi \\
0 & \sin \phi & \cos \phi
\end{array}\right]
$$

\section{Definition of Reference Frames}

Before discussing how the model of the landing gear system was constructed, the different reference frames must first be explained. These RFs are Earth (E), Body (B), Strut (S), and Turning Direction (T). The Earth reference frame serves as the basic reference frame. The Earth RF is inertial and the orientation of all the others is defined with respect to it. All the RFs used and the coordinate systems associated to them are described next. The reference angles are the attitude angles used in transforming the x-, $y$-, and z-axis coordinates from the base reference frame to the respective current reference frame counterparts.

\section{C1. Earth Reference Frame}

Notation: $\mathrm{RF}_{\mathrm{E}}$

Origin: $\mathrm{O}_{\mathrm{E}}$, the mass center of the Earth. 
System of Coordinates: $O_{E} X_{E} Y_{E} Z_{E}$

Orientation: $O_{E} Z_{E}$ axis is along the axis of Earth rotation, positive towards North.

$O_{E} X_{E}$ axis is defined by the center of the Earth $\mathrm{E}$ and the point of

intersection between the equator and the first meridian (Greenwich).

$O_{E} Y_{E}$ axis results according to the right-hand rule.

$\mathrm{RF}_{\mathrm{E}}$ is rotating with the Earth and also follows Earth revolution.

For most of the simulation, the flat Earth approximation will be used in which a modified Earth frame is defined with the origin at a reference location A on the surface of the Earth, $A Z_{E}$ normal to the surface positive towards the Earth, $A X_{E}$ along the projected meridian towards North, and $A Y_{E}$ according to the right-hand rule, will point towards the East. This is just a particular version of what is also called the geographic Coordinate System. A grid of lines of longitude (meridians) and latitude (parallels) determines any location on the surface of the Earth. Longitude varies between $\pm 180^{\circ}$. $0^{\circ}$ corresponds to the meridian through Greenwich. Positive longitude is considered eastwards. Latitude varies between $\pm 90^{\circ}$, it is measured from the Equator, positive to the North. The nautical mile is defined as the arc length of 1 minute. The origin may be considered at a reference location on the surface of the Earth, one axis points to the center of the Earth, the other two are tangent to the local meridian (positive to wards the North) and the parallel, respectively, as governed by the right-hand rule.

\section{C2. Body Reference Frame}

Notation: $\mathrm{RF}_{\mathrm{B}}$ 
Origin: $\mathrm{O}_{\mathrm{B}}$, the mass center of the vehicle. Note that for all simulation purposes the center of mass and center of gravity will be considered to coincide.

System of Coordinates: $O_{B} X_{B} Y_{B} Z_{B}$

Orientation: $\quad O_{B} X_{B}$ axis is along an axis that has an important "significance", positive

"forward", towards the "nose" of the vehicle. The "significance"

of the axis may be based on geometry (symmetry), inertial

properties (principal axes of the moment of inertia tensor), or

aerodynamics (stability axes).

$O_{B} Z_{B}$ axis belongs to the plane of symmetry, since all aerospace vehicles

have a plane of (almost) symmetry. It points downward.

$O_{B} Y_{B}$ axis results according to the right-hand rule.

Reference Angles from Base Frame: $[\varphi \theta \psi]$, these are the traditional Euler angles with the Earth as a base. They correspond to rotations from the Earth RF to the body RF by first rotating about the z-axis, then the y-axis, and finally the x-axis. This correlates to yawing by $\psi$ degrees, then pitching by $\theta$ degrees, and lastly rolling by $\varphi$ degrees. Converting from the Earth RF to the Body RF is depicted in Figure 1. The black axes are the original Earth axes. Rotating about the z-axis gives the blue axes. Next the red axes are created by rotating about the new y-axis. To get to the new body axes in green, rotate about the $\mathrm{x}$-axis. 


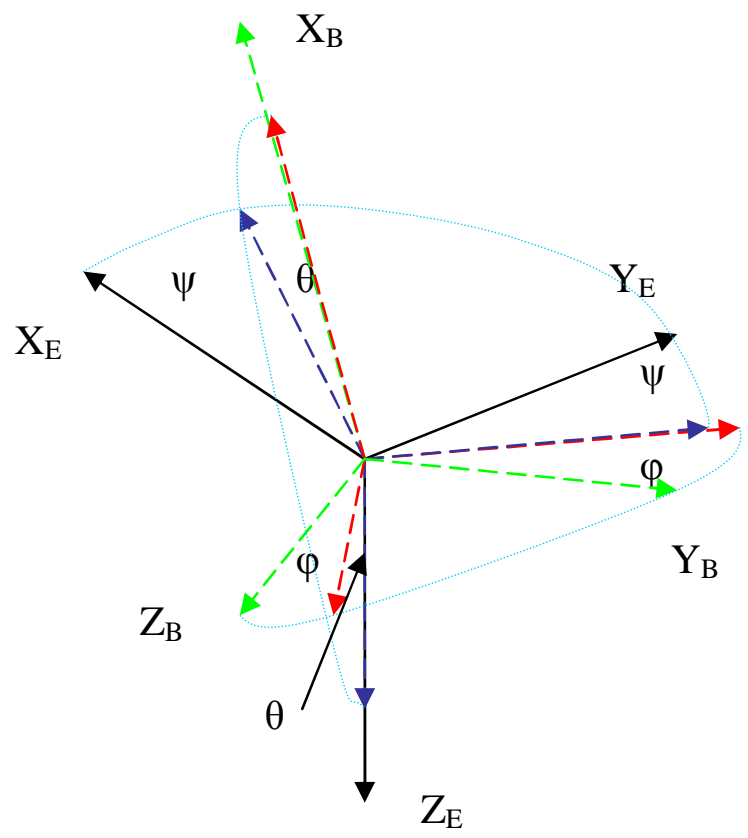

Figure 1. Depiction of changing from the Earth RF to the body RF

\section{C3. Strut Reference Frame}

Notation: $\mathrm{RF}_{\mathrm{S}}$

Origin: $\mathrm{O}_{\mathrm{S}}$, the horizontal plane of $\mathrm{RF}_{\mathrm{B}}$.

System of Coordinates: $O_{S} X_{S} Y_{S} Z_{S}$

Orientation: $\quad O_{S} X_{S}$ axis lies in the $O_{B} X_{B}, O_{B} Z_{B}$ plane, orthogonal to $O_{S} Z_{S}$ in the direction of $O_{B} X_{B}$

$O_{S} Z_{S}$ axis is along the virtual strut direction, for simulation purposes all strut deflection occurs along this axis

$O_{S} Y_{S}$ axis results according to the right-hand rule. 
Reference Angles from Base Frame: $\left[\varphi_{S} \theta_{S} \psi_{S}\right]$, where the Base frame is the Body RF. The conversion from the body RF to the strut RF mimics the conversion from the Earth RF to the body RF. Rotate $\psi_{\mathrm{S}}$ degrees about the z-axis, then $\theta_{\mathrm{S}}$ degrees about the z-axis, and end with rotating $\varphi_{S}$ degrees about the $\mathrm{x}$-axis. This can be seen below in Figure 2 , by going from black to blue to red to green.

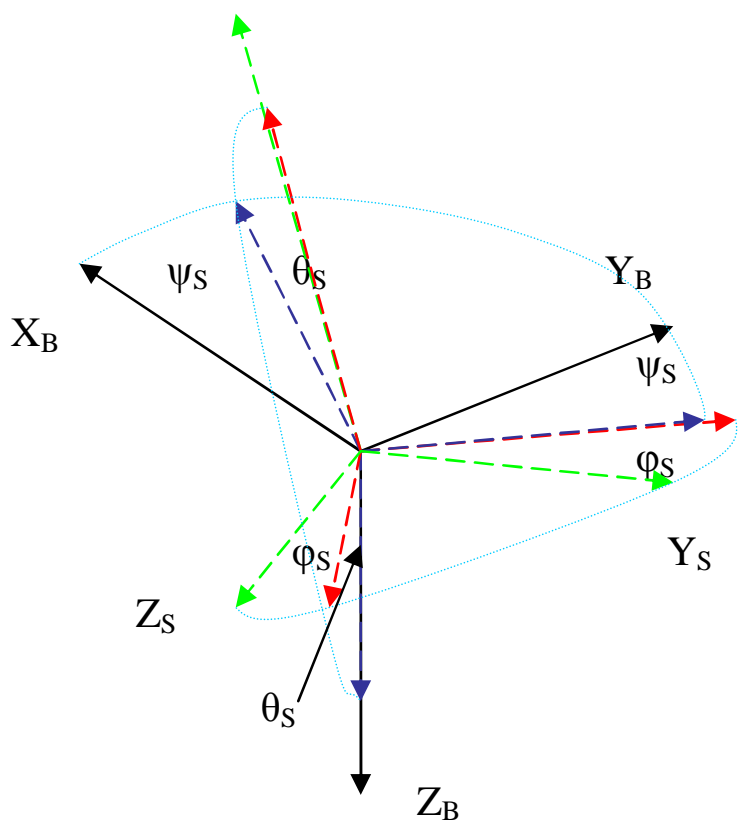

Figure 2. Depiction of changing from the body RF to the strut RF

\section{C4. Turning Reference Frame}

Notation: $\mathrm{RF}_{\mathrm{T}}$

Origin: $\mathrm{O}_{\mathrm{T}}$, the mass center of the vehicle.

System of Coordinates: $O_{T} X_{T} Y_{T} Z_{T}$ 
Orientation: $O_{T} X_{T}$ axis is along an axis in the rolling direction of the steering capable landing gear, orthogonal to $O_{T} Z_{T}$ $O_{T} Z_{T}$ axis is parallel to $O_{S} Z_{S}$ $O_{T} Y_{T}$ results according to the right-hand rule.

Reference Angles from Base Frame: $\left[\begin{array}{lll}0 & 0 & \psi_{\mathrm{T}}\end{array}\right]$, where the Base frame is the Strut Reference Frame. Rotating $\psi_{\mathrm{T}}$ degrees about the z-axis completes the conversion from the strut RF to the turning RF. Seen below in Figure 3 is this transformation as going from the black axes to the blue ones.

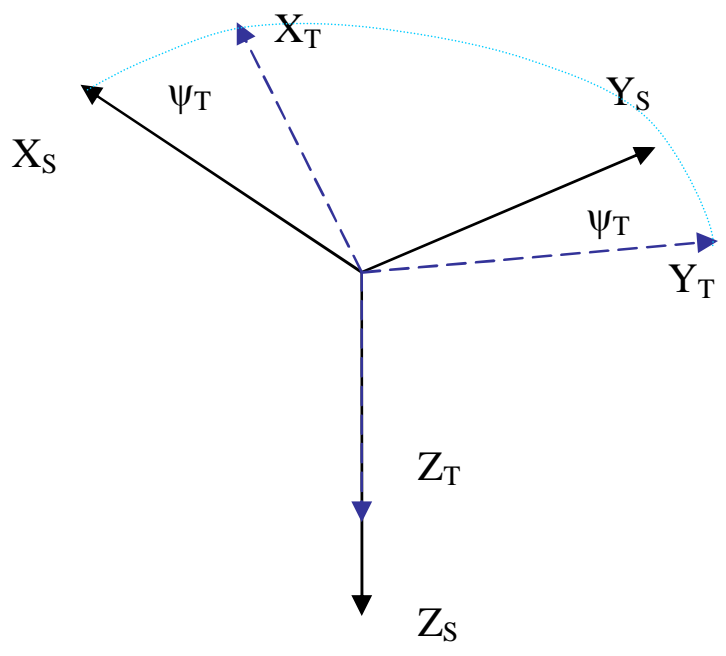

Figure 3. Depiction of changing from the strut RF to the turning RF

Figure 4 shows all of the different possible axes simultaneously projected onto a drawing of an aircraft. The $\mathrm{O}_{\mathrm{E}}$ location is a reference point located on the surface of the Earth as denoted by the dashed plane. The dotted axis lines occur when the axis are found within the body of the aircraft to aid in the perception of the axes. The blue dashed lines are pointers used to connect the label to the point or angle of interest to that 
component. Since the aircraft is off of the ground, the aircraft cannot turn by input from the landing gear. This in turn implies that the turning reference and the strut reference frame overlap. The main strut reference frame is not shown to make the diagram easier to be understood. These can be inserted to the diagram by placing a reference frame similar to the nose strut at the top of the main struts. Additionally shown is the projection of the $\mathrm{RF}_{\mathrm{E}}$ on the aircraft. The transformation from this axis system to the body axis system is shown, by transferring from the black axis, to the blue one, to the red, and finally to the green. This transformation is done by rotating by the Euler angles.

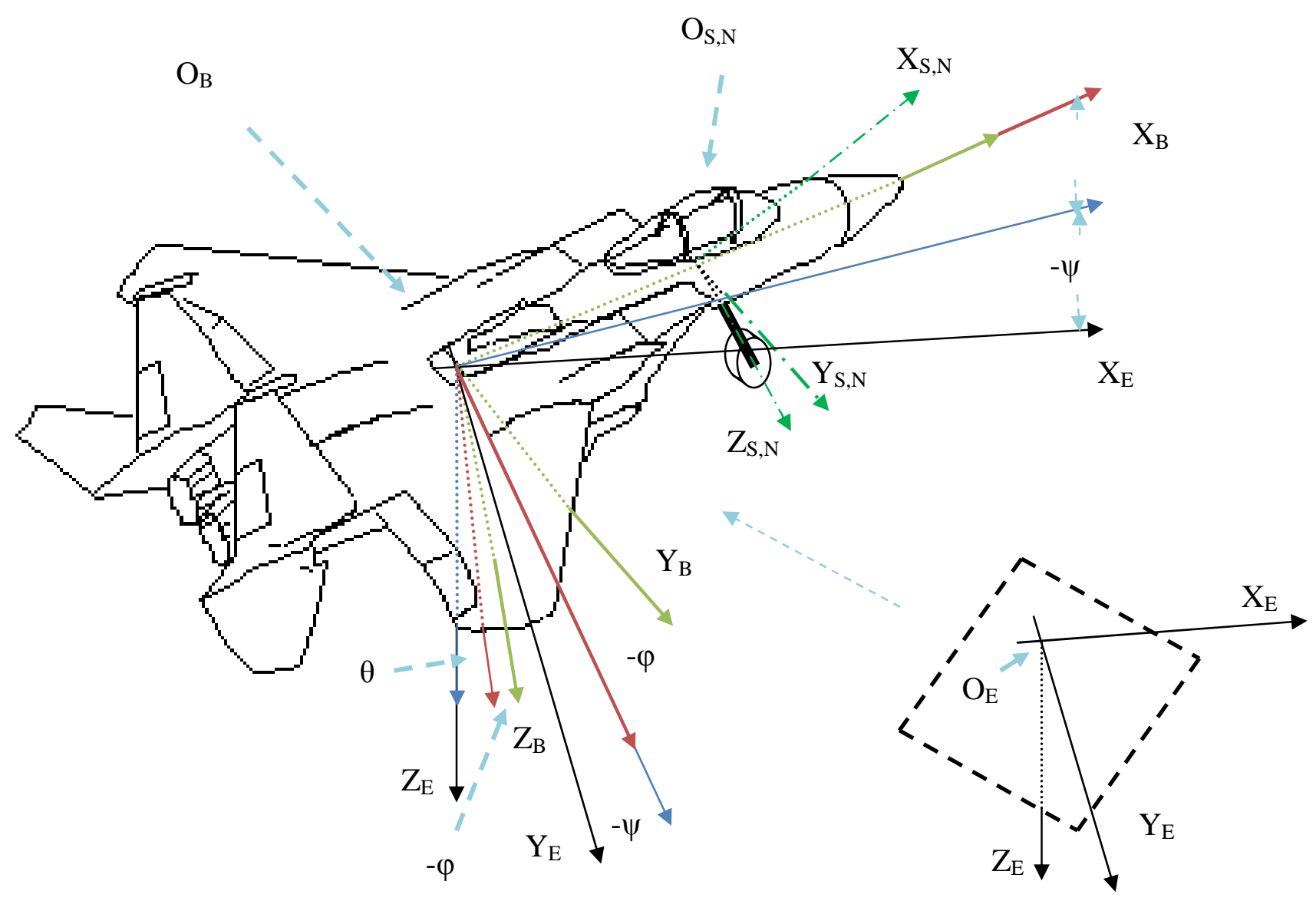

Figure 4. Depiction of the RF's on an aircraft body. 


\section{Integration of the Aircraft Motion and Landing Gear Models}

The current methodology for flight simulation is to use the fifteen equations of flight modeling. These equations are the six coupled equations involved with the Conservation of Linear and Angular Momentum (CLME and CAME, respectively), three Gravity Equations, three Euler angle conversion equations (known as Kinematics), and three Earth Position equations ${ }^{[26]}$ or Trajectory Equations. The logical flow for how these equations interact with each other is depicted in Figure 5. The equations themselves are listed as Equations (2.11) to (2.15).

Each equation is paramount to defining the position of the aircraft relative to the Earth. Solving the coupled equations, CLME and CAME, generate the body linear velocities relative to the air along the $\mathrm{x}_{-}, \mathrm{y}-$ and $\mathrm{z}-$ axis $(\mathrm{U}, \mathrm{V}$, and $\mathrm{W}$, respectively) and the rotational velocities along those same axes $(\mathrm{P}, \mathrm{Q}$, and $\mathrm{R}$, respectively). These rotational velocities are then used in solving the Kinematics Equations to find the aircraft's Euler angles $(\Phi, \Theta$, and $\Psi)$. These angles are used to transform the linear velocities of the aircraft given in the body reference frame to the Earth reference frame, which in turn give the position of the aircraft relative to the origin of the Earth. For the position of the aircraft to be known, the velocity of the wind relative to the Earth must be known, $\left[\begin{array}{lll}U_{\text {wind }} & V_{\text {wind }} & W_{\text {wind }}\end{array}\right]_{E}^{T}$. The Euler angles are also used in calculating the components of the gravitational force into the body reference frame. Triplets $(\mathrm{U}, \mathrm{V}, \mathrm{W}),(\mathrm{P}, \mathrm{Q}, \mathrm{R}),\left(\mathrm{X}_{\mathrm{E}}\right.$, $\left.\mathrm{Y}_{\mathrm{E}}, \mathrm{Z}_{\mathrm{E}}\right)$, and $(\Phi, \Theta, \Psi)$ form what is typically known as the states of the aerodynamic algorithm. 


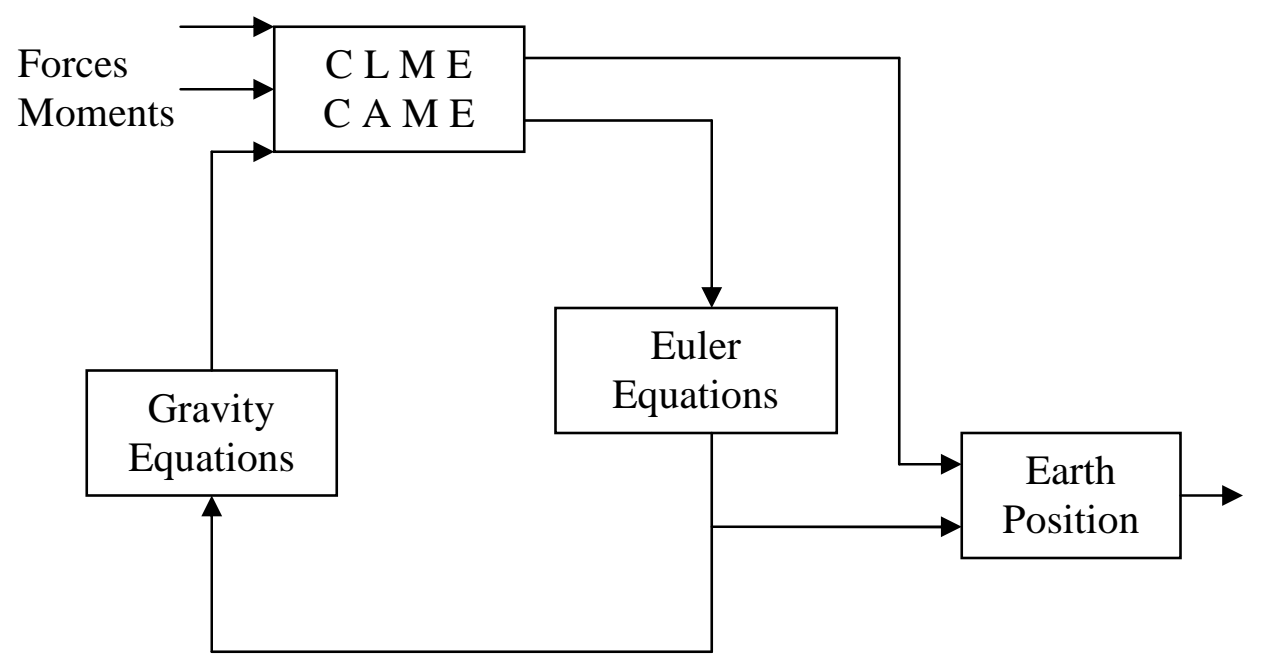

Figure 5. General diagram of a flight simulation algorithm

\section{CLME}

$m(\dot{U}-V R+W Q)=m g_{x}+F_{x, \text { Total }}$

$m(\dot{V}+U R-W P)=m g_{y}+F_{y, \text { Total }}$

$m(\dot{W}-U Q+V P)=m g_{z}+F_{z, \text { Total }}$

\section{CAME}

$I_{x x} \dot{P}-I_{x z} \dot{R}-I_{x z} P Q+\left(I_{z z}-I_{y y}\right) R Q=M_{x, \text { Total }}$

$I_{y y} \dot{Q}+\left(I_{x x}-I_{z z}\right) P R+I_{x z}\left(P^{2}-R^{2}\right)=M_{y, \text { Total }}$

$I_{z z} \dot{R}-I_{x z} \dot{P}+\left(I_{y y}-I_{x x}\right) P Q+I_{x z} Q R=M_{z, \text { Total }}$

\section{Euler Equations}

$\dot{\Phi}=P+Q \sin \Phi \tan \Theta+R \cos \Phi \tan \Theta$

$\dot{\Theta}=Q \cos \Phi-R \sin \Phi$

$\dot{\Psi}=(Q \sin \Phi+R \cos \Phi) \sec \Theta$

\section{Gravity Equations}

$g_{x}=-g \sin \Theta$

$g_{y}=g \sin \Phi \cos \Theta$

$g_{z}=g \cos \Phi \cos \Theta$ 


\section{Earth Position}

$$
\begin{aligned}
\dot{X}_{E}= & \left\{\left(U+U_{\text {wind }}\right) \cos \Theta+\left[\left(V+V_{\text {wind }}\right) \sin \Phi+\left(W+W_{\text {wind }}\right) \cos \Phi\right] \sin \Theta\right\} \cos \Psi \ldots \\
& -\left[\left(V+V_{\text {wind }}\right) \cos \Phi-\left(W+W_{\text {wind }}\right) \sin \Phi\right] \sin \Psi \\
\dot{Y}_{E}= & \left\{\left(U+U_{\text {wind }}\right) \cos \Theta+\left[\left(V+V_{\text {wind }}\right) \sin \Phi+\left(W+W_{\text {wind }}\right) \cos \Phi\right] \sin \Theta\right\} \sin \Psi \ldots \\
& +\left[\left(V+V_{\text {wind }}\right) \cos \Phi-\left(W+W_{\text {wind }}\right) \sin \Phi\right] \cos \Psi \\
H_{E}= & \left(U+U_{\text {wind }}\right) \sin \Theta-\left[\left(V+V_{\text {wind }}\right) \sin \Phi+\left(W+W_{\text {wind }}\right) \cos \Phi\right] \cos \Theta
\end{aligned}
$$

As can be seen, the only input to the system comes in the way of the total forces and moments applied in the body reference frame. The output is the position vector of the Center of Gravity of the aircraft with respect to the Earth reference frame, $\overrightarrow{O_{E}} C \vec{G}$, given in components with respect to $R F_{E}$ as $\left[\overrightarrow{O_{E} C G}\right]_{E}=\left[\begin{array}{lll}O_{E} C G_{X} & O_{E} C G_{Y} & O_{E} C G_{Z}\end{array}\right]_{E}^{T}$. In flight, the total force would just be that component created by aerodynamic and thrust effects. To add input from the Landing Gear system, the total force and moment equations should be as follows:

$$
\begin{aligned}
& F_{x, \text { Total }}=F_{x, \text { Aero }}+F_{x, \text { Thrust }}+F_{x, L G} \\
& F_{y, \text { Total }}=F_{y, \text { Aero }}+F_{y, \text { Thrust }}+F_{y, L G} \\
& F_{z, \text { Total }}=F_{z, \text { Aero }}+F_{z, \text { Thrust }}+F_{z, L G} \\
& M_{x, \text { Total }}=M_{x, \text { Aero }}+M_{x, \text { Thrust }}+M_{x, L G} \\
& M_{y, \text { Total }}=M_{y, \text { Aero }}+M_{y, \text { Thrust }}+M_{y, L G} \\
& M_{z, \text { Total }}=M_{z, \text { Aero }}+M_{z, \text { Thrust }}+M_{z, L G}
\end{aligned}
$$

With this in mind, the output from the landing gear modeling system should be the total forces and moments the landing system generates. To find these forces and moments a three part algorithm must be followed consisting of: Force Generation, Length of Landing Gear Finding, and Friction Calculation. 


\section{D1. Force Generation}

To determine the force generated by the landing gear system, the dynamic model of the landing gear system needs to be examined. The dynamic model for a landing gear system composed of an oleo and tire can be modeled as a spring-damper system as pictured below in Figure 6 with common labeling practices. This type of model allows for three different variables to be assigned to the system, namely the spring and damper coefficients of the oleo along with the spring coefficient of the tire.

The $\mathrm{Z}$ notation is used for end points corresponding to the location of components of the aircraft vital in tracking the dynamics of the landing gear model. These points determine the distances between where the strut intersects the aircraft fuselage $Z_{a}$, also known as the origin of the strut reference frame, $\mathrm{O}_{\mathrm{S}}$, the hub of the wheel $Z_{w}$, and the contact point to the ground $Z_{g}$. The distance from $Z_{a}$ to $Z_{w}$ is defined by the position vector $\overrightarrow{Z_{a} Z_{w}}$. The magnitude of this vector is denoted by $\left|\overrightarrow{Z_{a} Z_{w}}\right|$. Following this notation convention presented in the previous section, $\left|\overrightarrow{Z_{w} Z_{g}}\right|$ gives the magnitude of the distance between $Z_{w}$ and $Z_{g}$. Since $Z_{a}$ is attached to the rigid body of the aircraft and the strut reference frame is constant relative to the body, the position of $Z_{a}$ is constant. Additionally, it follows that $\left(\dot{\overrightarrow{O_{s} Z_{a}}}\right)_{S}$ is zero. $F_{\text {oleo }}$ is the force acting on the body of the aircraft which is generated by the oleo. $F_{\text {tire }}$ is the force generated by the tire which acts on the Earth. Figure 7 demonstrates this dynamic model applied to a linear strut. 

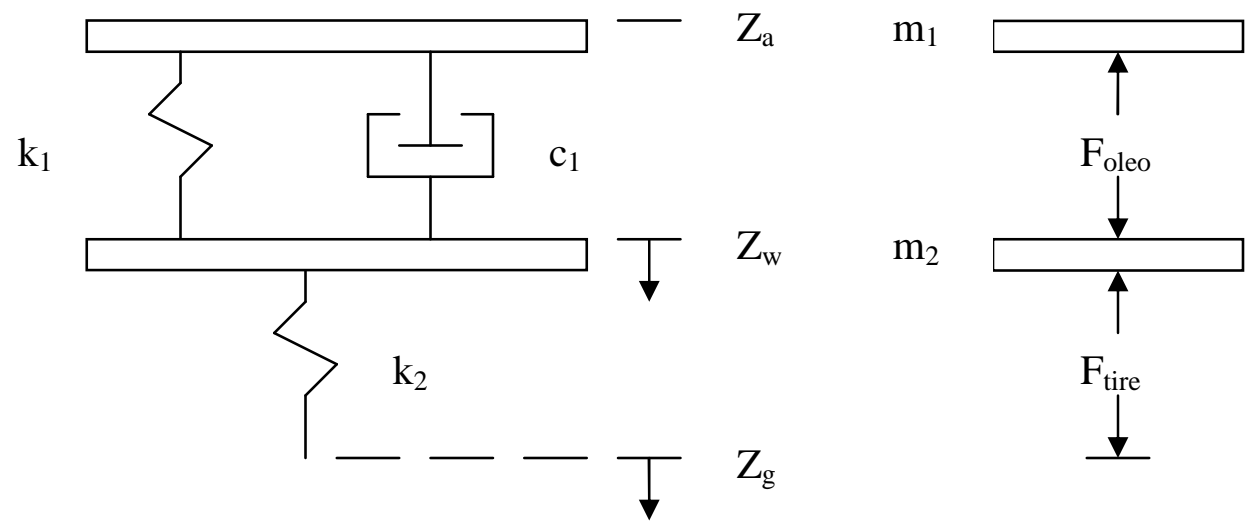

Figure 6. Dynamic model of a landing gear strut with free body diagram.

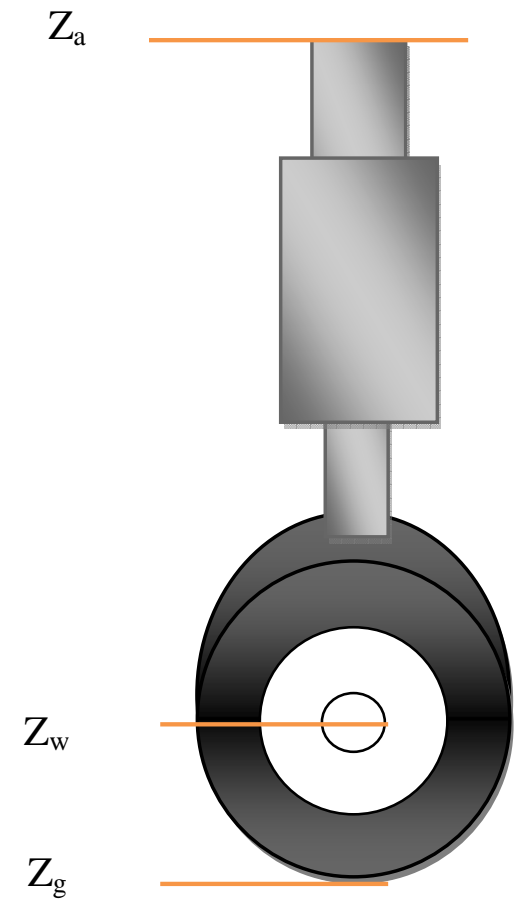

Figure 7. Points of interest dynamically applied to a linear strut. 
From this model, the following three equations can be deduced using Modern Control Engineering as a reference ${ }^{[27]}$. In defining these equations, $g_{z, S}$ is the component of gravity along the SDL.

$$
\begin{aligned}
& F_{\text {oleo }}=-k_{1}\left(\left|\overrightarrow{Z_{a} Z_{w}}\right|-\left|\overrightarrow{Z_{a} Z_{w, \text { unloaded }}}\right|\right)-c_{1}\left(\left|\overrightarrow{Z_{a} Z_{w}}\right|\right)_{S} \\
& F_{\text {tire }}=k_{2}\left(\operatorname{Rad}_{\text {tire }}-\left(\left|\overrightarrow{Z_{a} Z_{g}}\right|-\left|\overrightarrow{Z_{a} Z_{w}}\right|\right)\right) \\
& m_{2}\left(\left|\overrightarrow{Z_{a} Z_{w}}\right|\right)_{S}=F_{\text {oleo }}-F_{\text {tire }}+m_{2} g_{z, S}
\end{aligned}
$$

These equations hold true as long as the difference between $\left|\overrightarrow{Z_{a} Z_{g}}\right|$ and $\left|\overrightarrow{Z_{a} Z_{w}}\right|$ is less than the original radius of the tire, $\operatorname{Rad}_{\text {tire }}$. When the distance is greater than the tire radius, the force in the tire is zero. Additionally, there is a threshold force that the tire must provide before the oleo begins to operate. With these two alternative equations in mind, the force equations are expressed below.

$$
\begin{gathered}
F_{\text {oleo }}=\left\{\begin{array}{lr}
-k_{1}\left(\left|\overrightarrow{Z_{a} Z_{w}}\right|-\left|\overrightarrow{Z_{a} Z_{w, \text { unloaded }}}\right|\right)-c_{1}\left(\left|\overrightarrow{Z_{a} Z_{w}}\right|\right)_{S} \text { for } F_{\text {tire }} \geq F_{\text {oleo, threshold }} \\
F_{\text {tire }} & \text { for } F_{\text {tire }}<F_{\text {oleo,threshold }}
\end{array}\right. \\
F_{\text {tire }}=\left\{\begin{array}{lr}
k_{2}\left(\operatorname{Rad}_{\text {tire }}-\left(\left|\overrightarrow{Z_{a} Z_{g}}\right|-\left|\overrightarrow{Z_{a} Z_{w}}\right|\right)\right) & \text { for }\left(\left|\overrightarrow{Z_{a} Z_{w}}\right|-\left|\overrightarrow{Z_{a} Z_{g}}\right|\right)<\operatorname{Rad}_{\text {tire }} \\
0 & \text { for }\left(\left|\overrightarrow{Z_{a} Z_{w}}\right|-\left|\overrightarrow{Z_{a} Z_{g}}\right|\right) \geq \operatorname{Rad}_{\text {tire }}
\end{array}\right.
\end{gathered}
$$

To apply these equations, $\left|\overrightarrow{Z_{a} Z_{w}}\right|$ must be tracked. This is done by using the equation containing $\left(\left|\overrightarrow{Z_{a} Z_{w}}\right|\right)_{S}$. As seen below, rearranging this equation and integrating, (using the Runga-Kutta method), yields the equations for the wheel velocity along the SDL, 
$\left(\left|\overrightarrow{Z_{a} Z_{w}}\right|\right)_{S}$, and the distance between the fuselage and the wheel, once it is integrated again.

$$
\begin{aligned}
& \left(\left|\overrightarrow{Z_{a} Z_{w}}\right|\right)_{S}=\int\left[\frac{1}{m_{2}} F_{\text {oleo }}-\frac{1}{m_{2}} F_{\text {tire }}+g_{z, S}\right] \\
& \left|\overrightarrow{Z_{a} Z_{w}}\right|=\int\left(\left|\overrightarrow{Z_{a} Z_{w}}\right|\right)_{S}
\end{aligned}
$$

As was mentioned earlier, these modeling equations all depend on knowing the value for $\left|\overrightarrow{Z_{a} Z_{g}}\right|$.

\section{D2. Determining the Length of the Landing Gear}

The distance from $Z_{a}$ to the ground along the SDL, $\left|\overrightarrow{Z_{a} Z_{g}}\right|$, must be known. This can be accomplished by completing a simple algorithm involving vector transformations. The first step is to find the position vector of the connection point in the Earth reference frame origin, $\overrightarrow{O_{E} Z_{a}}$. As was noted earlier, the position of the aircraft's $C G, \mathrm{O}_{\mathrm{B}}$, relative to the Earth, $\overrightarrow{O_{E} C G}$, is always known within the simulated environment. Since the aircraft is assumed to be a rigid structure, the distance from the CG to $Z_{a}, \overrightarrow{C G Z_{a}}$, is constant.

To change this to the necessary $\left[\overrightarrow{C G Z_{a}}\right]_{E},\left[\overrightarrow{C G Z_{a}}\right]_{B}$ needs to be multiplied by the transformation matrix given below where the angles used in the equation are $[\varphi \theta \psi]$ as noted above. By adding $\overrightarrow{C G Z_{a}}$ to $\overrightarrow{O_{E} C G}, \overrightarrow{O_{E} Z_{a}}$ can be found. 


$$
\begin{aligned}
& {\left[\overrightarrow{C G Z_{a}}\right]_{E}=\left(\begin{array}{ccc}
\cos \psi & -\sin \psi & 0 \\
\sin \psi & \cos \psi & 0 \\
0 & 0 & 1
\end{array}\right)\left(\begin{array}{ccc}
\cos \theta & 0 & \sin \theta \\
0 & 1 & 0 \\
-\sin \theta & 0 & \cos \theta
\end{array}\right)\left(\begin{array}{ccc}
1 & 0 & 0 \\
0 & \cos \phi & -\sin \phi \\
0 & \sin \phi & \cos \phi
\end{array}\right) \cdot\left[\overrightarrow{C G Z_{a}}\right]_{B}} \\
& \overrightarrow{O_{E} Z_{a}}=\overrightarrow{C G Z_{a}}+\overrightarrow{O_{E} C G}
\end{aligned}
$$

The second step is to deduce the vector for the virtual SDL. Accomplishing this goal is again achieved through vector transformations. Since it is assumed that the strut only acts parallel to the $\mathrm{z}$-axis of the strut reference frame, the unit vector for the virtual SDL in the strut reference frame is given by $\left[\begin{array}{lll}0 & 0 & 1\end{array}\right]_{S}^{T}$. This vector can then be transformed into the Earth reference frame, $[\overrightarrow{S D L}]_{E}$, by transforming it first to the body frame and then finally the Earth frame by using the rotation angles $\left[\varphi_{\mathrm{S}}, \theta_{\mathrm{S}}, \psi_{\mathrm{S}}\right]$ and $[\varphi, \theta, \psi]$, respectively, in the transformation equation as shown below.

$$
\begin{aligned}
& {[\overrightarrow{S D L}]_{B}=\left(\begin{array}{ccc}
\cos \psi_{S} & -\sin \psi_{S} & 0 \\
\sin \psi_{S} & \cos \psi_{S} & 0 \\
0 & 0 & 1
\end{array}\right)\left(\begin{array}{ccc}
\cos \theta_{S} & 0 & \sin \theta_{S} \\
0 & 1 & 0 \\
-\sin \theta_{S} & 0 & \cos \theta_{S}
\end{array}\right)\left(\begin{array}{ccc}
1 & 0 & 0 \\
0 & \cos \phi_{S} & -\sin \phi_{S} \\
0 & \sin \phi_{S} & \cos \phi_{S}
\end{array}\right)\left[\begin{array}{l}
0 \\
0 \\
1
\end{array}\right]_{S}} \\
& {[\overrightarrow{S D L}]_{E}=\left(\begin{array}{ccc}
\cos \psi & -\sin \psi & 0 \\
\sin \psi & \cos \psi & 0 \\
0 & 0 & 1
\end{array}\right)\left(\begin{array}{ccc}
\cos \theta & 0 & \sin \theta \\
0 & 1 & 0 \\
-\sin \theta & 0 & \cos \theta
\end{array}\right)\left(\begin{array}{ccc}
1 & 0 & 0 \\
0 & \cos \phi & -\sin \phi \\
0 & \sin \phi & \cos \phi
\end{array}\right)[\overrightarrow{S D L}]_{B}}
\end{aligned}
$$

Lastly, the location of the Ground Contact Point, GCP, where the extension of the SDL intersects the surface of the Earth, must be found. To find this point, the parametric equation for the SDL extension is expressed as ${ }^{[28]} \vec{r}=\vec{a}+A R V \vec{b}$. In this equation, $\vec{r}$ is 
the position vector of a point on the extension, $\vec{a}$ is a position vector of a reference point on SDL and $\vec{b}$ is a unit vector along the line, SDL. $A R V$ is an arbitrary real value that is used to define the set of points. By changing the value of ARV, the set of points that the line contains is created. The position vector to be used is the one created between the origin of the Earth and the fuselage connection point, $\left[\overrightarrow{O_{E} Z_{a}}\right]_{E}$. The vector used in this equation is $[\overrightarrow{S D L}]_{E}$. This gives the following equation:

$$
[\vec{r}]_{E}=\left[\overrightarrow{O_{E} Z_{a}}\right]_{E}+A R V[\overrightarrow{S D L}]_{E}
$$

Rewriting this equation with $[\overrightarrow{S D L}]_{E}$ defined as $\left[\begin{array}{lll}S D L_{x} & S D L_{y} & S D L_{z}\end{array}\right]_{E}^{T},\left[\overrightarrow{O_{E} Z_{a}}\right]_{E}$

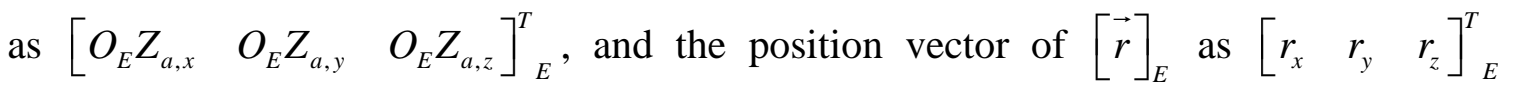
yields the system of equations for the virtual SDL in the Earth reference frame.

$$
\left\{\begin{array}{l}
O_{E} Z_{a, x}+A R V\left(S D L_{x}\right)=r_{x} \\
O_{E} Z_{a, y}+A R V\left(S D L_{y}\right)=r_{y} \\
O_{E} Z_{a, z}+A R V\left(S D L_{z}\right)=r_{z}
\end{array}\right\}_{E}
$$

Using this system of equations and an additional equation governing the surface of the runway, the Ground Contact Point can be found. The equation for a planar surface is given by $A x+B y+C z=D$, where $\mathrm{x}, \mathrm{y}$, and $\mathrm{z}$ are the components of the position vector of a point on that surface. For a truly precise model of landing gear simulation, this equation needs to be recalculated for each time step of the program. In the case of the landing surface being modeled as a flat horizontal surface with altitude alt, which is 
done in this model, the following governing equation is used. If roughness in the landing surface is to be included, adding it to the value for alt in each calculation can be used to model this behavior.

$$
\begin{aligned}
& 0 \cdot x+0 \cdot y+z=a l t \\
& \rightarrow z=\text { alt }
\end{aligned}
$$

By rearranging the equations (2.24) and (2.25) the following matrix equation can be constructed. Since the $\left[\overrightarrow{O_{E} G C P}\right]_{E}$ is a possible solution for $[\vec{r}]_{E}$ the component version of $\overrightarrow{O_{E} G C P},\left[\begin{array}{lll}O_{E} G C P_{x} & O_{E} G C P_{y} & O_{E} G C P\end{array}\right]_{E}$, can replace $\left[\begin{array}{lll}r_{x} & r_{y} & r_{z}\end{array}\right]_{E}$ in the equation. A diagram is presented as Figure 8 with $\left[\overrightarrow{O_{E} G C P}\right]_{E}$ being a point on the horizontal plane and the orange line segment being $[\overrightarrow{S D L}]_{E}$.

$$
\left[\begin{array}{cccc}
1 & 0 & 0 & -S D L_{x} \\
0 & 1 & 0 & -S D L_{y} \\
0 & 0 & 1 & -S D L_{z} \\
0 & 0 & 1 & 0
\end{array}\right]_{E}\left[\begin{array}{c}
O_{E} G C P_{x} \\
O_{E} G C P_{y} \\
O_{E} G C P_{z} \\
A R V
\end{array}\right]_{E}=\left[\begin{array}{c}
O_{E} Z_{a, x} \\
O_{E} Z_{a, y} \\
O_{E} Z_{a, z} \\
\text { alt }
\end{array}\right]_{E}
$$

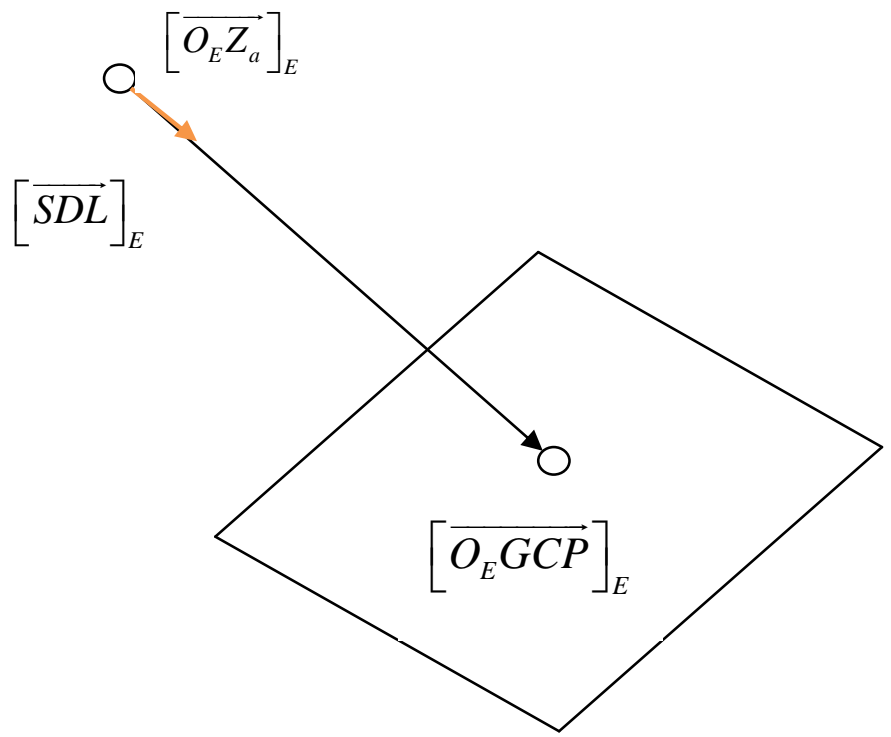

Figure 8. Depiction of geometric components used in determining strut length 
The main reason for knowing the $\vec{O}_{E} Z_{a}$ position vector and the $\overrightarrow{O_{E} G C P}$ position vector is to know the magnitude of the distance between them. Using any of a number of ways to find the solution to Equation 2.26, the next Equation can be created. Then the distance between the two points can be found with the following equation.

$$
\left|\overrightarrow{Z_{a} Z_{g}}\right|=\sqrt{\left(O_{E} G C P_{x}-O_{E} Z_{a, x, E}\right)^{2}+\left(O_{E} G C P_{y}-O_{E} Z_{a, y, E}\right)^{2}+\left(O_{E} G C P_{z}-O_{E} Z_{a, z, E}\right)^{2}}
$$

However, there is a more efficient technique in finding this magnitude, once the equation for SDL in the Earth reference frame is re-examined. By rearranging Equation (2.26) as follows, it is discovered that only $A R V$ needs to be found.

$$
\begin{aligned}
& A R V\left(S D L_{x}\right)=O_{E} G C P_{x}-O_{E} Z_{a, x} \\
& A R V\left(S D L_{y}\right)=O_{E} G C P_{y}-O_{E} Z_{a, y} \\
& A R V\left(S D L_{z}\right)=O_{E} G C P_{z}-O_{E} Z_{a, z} \\
& \left|\overline{Z_{a} Z_{g}}\right|=\sqrt{\left(A R V\left(S D L_{x, E}\right)\right)^{2}+\left(A R V\left(S D L_{y, E}\right)\right)^{2}+\left(A R V\left(S D L_{z, E}\right)\right)^{2}} \\
& \left|\overline{Z_{a} Z_{g}}\right|=\sqrt{A R V^{2}\left(S D L_{x, E}\right)^{2}+A R V^{2}\left(S D L_{y, E}\right)^{2}+A R V^{2}\left(S D L_{z, E}\right)^{2}} \\
& \left|\overline{Z_{a} Z_{g}}\right|=\sqrt{A R V^{2}\left\{\left(S D L_{x, E}\right)^{2}+\left(S D L_{y, E}\right)^{2}+\left(S D L_{z, E}\right)^{2}\right\}} \\
& \left|\overline{Z_{a} Z_{g}}\right|=A R V \cdot \sqrt{\left(S D L_{x, E}\right)^{2}+\left(S D L_{y, E}\right)^{2}+\left(S D L_{z, E}\right)^{2}}
\end{aligned}
$$

Examining this last equation, it is shown that $\left|\overrightarrow{Z_{a} Z_{g}}\right|$ is simply the product of the calculated value of $A R V$ and the magnitude of SDL. Using Cramer's Method the value of $A R V$ is calculated with the following equation: 


$$
A R V=\frac{\operatorname{det}\left[\begin{array}{cccc}
1 & 0 & 0 & O_{E} Z_{a, x} \\
0 & 1 & 0 & O_{E} Z_{a, y} \\
0 & 0 & 1 & O_{E} Z_{a, z} \\
0 & 0 & 1 & \text { alt }
\end{array}\right]_{E}}{\operatorname{det}\left[\begin{array}{cccc}
1 & 0 & 0 & -S D L_{x} \\
0 & 1 & 0 & -S D L_{y} \\
0 & 0 & 1 & -S D L_{z} \\
0 & 0 & 1 & 0
\end{array}\right]_{E}}
$$

Therefore, to find $\left|\overrightarrow{Z_{a} Z_{g}}\right|$ that was previously noted without the arbitrary variable $A R V$ the following equation is used.

$$
\mid \overrightarrow{Z_{a} Z_{g} \mid}=\sqrt{S D L_{x, E}^{2}+S D L_{y, E}^{2}+S D L_{z, E}^{2}} \frac{\operatorname{det}\left[\begin{array}{cccc}
1 & 0 & 0 & O_{E} Z_{a, x} \\
0 & 1 & 0 & O_{E} Z_{a, y} \\
0 & 0 & 1 & O_{E} Z_{a, z} \\
0 & 0 & 1 & \text { alt }
\end{array}\right]_{E}}{\operatorname{det}\left[\begin{array}{cccc}
1 & 0 & 0 & -S D L_{x} \\
0 & 1 & 0 & -S D L_{y} \\
0 & 0 & 1 & -S D L_{z} \\
0 & 0 & 1 & 0
\end{array}\right]_{E}}
$$

Since $\overrightarrow{S D L}$ is generated from a unit vector, the magnitude of $\overrightarrow{S D L}$ is one. From this it is seen that the magnitude of $\left|\overrightarrow{Z_{a} Z_{g}}\right|$ is $A R V$.

$$
\begin{aligned}
&\left|\overrightarrow{Z_{a} Z_{g}}\right|=A R V \\
&\left|\overrightarrow{Z_{a} Z_{g}}\right| \operatorname{det}\left[\begin{array}{cccc}
1 & 0 & 0 & O_{E} Z_{a, x} \\
0 & 1 & 0 & O_{E} Z_{a, y} \\
0 & 0 & 1 & O_{E} Z_{a, z} \\
0 & 0 & 1 & \text { alt }
\end{array}\right]_{E} \\
& \operatorname{det}\left[\begin{array}{cccc}
1 & 0 & 0 & -S D L_{x} \\
0 & 1 & 0 & -S D L_{y} \\
0 & 0 & 1 & -S D L_{z} \\
0 & 0 & 1 & 0
\end{array}\right]_{E}
\end{aligned}
$$


In the case of a horizontal landing surface, as this is, the equation can be further simplified. This simplified equation is as follows:

$$
\left|\overrightarrow{Z_{a} Z_{g}}\right|=\frac{a l t-O_{E} Z_{a, z, E}}{S D L_{z, E}}
$$

\section{D3. Friction Calculation}

For calculations of the effects of landing gear on the aircraft, forces and moments corresponding to the $\mathrm{O}_{S} \mathrm{X}_{S} \mathrm{Y}_{S} Z_{S}$ directions are applied at $Z_{a}$. So far, only the force in the $Z_{S}$ direction has been calculated. This force is the same as that created by the oleo. To find the other two forces, the force transmitted through the tire is used as a starting point. The tire force, once normalized, is multiplied by a friction factor to calculate the maximum frictional force. This maximum friction force is only applied when the tire is moving or when the forces acting on the tire are greater than the maximum frictional force. Cases when less than maximum frictional force is needed will be explored later.

A tire's movement relative to the landing surface can be broken down into two different modes, rolling and sliding. These modes of locomotion occur in the $\mathrm{X}_{\mathrm{T}}$ and $\mathrm{Y}_{\mathrm{T}}$ axes, respectively. Instead of using the equation for rolling friction, the rolling frictional force of the tire is modeled as though it was a sliding frictional force with an alterable friction coefficient. This greatly lowers the complexity of the model without any large implications on the system since the equation for rolling frictional force is dependent on velocity, temperature, rubber characteristics, and other conditions according to Engineering Mechanics Statics $^{[29]}$. To calculate sliding friction, the sliding force is equivalent to the normal force multiplied by a friction factor. The base friction factor, $\mu_{\text {rolling }}$, was found by comparing against proprietary takeoff data. By using a variable, 
$\mu_{\text {Brake }}$, alterations to the rolling friction coefficient can be made. By adding $\mu_{\text {Brake }}$, braking capabilities are modeled. The higher $\mu_{\text {Brake }}$ is the more force the pilot is applying to the brakes. It should be noted that $\left(\mu_{\text {rolling }}+\mu_{\text {Brake }}\right)$ maxes out at $\mu_{\text {kinetic }}$. At this maximum condition, the tire is no longer rolling in the $\mathrm{X}_{\mathrm{T}}$ direction but is now sliding as well. The Engineering Handbook gives $\mu_{\text {kinetic }}$ to be between .5 and $.8^{[30]}$.

$$
\begin{aligned}
& F_{\text {friction,Max }}=f_{\text {friction }} \cdot F_{\text {Normal }} \\
& \therefore f_{\text {friction }}=\left\{\begin{array}{cc}
\left(\mu_{\text {rolling }}+\mu_{\text {Brake }}\right) & X-\text { Axis } \\
\mu_{\text {kinetic }} & Y-A x i s
\end{array}\right.
\end{aligned}
$$

Since a method for determining the friction factor has been deduced, the force normal to the local landing surface must be found. The normal force is the component of the applied force, $F_{\text {Tire }}$, perpendicular to the plane. To find $F_{\text {Normal }}$, the local surface of the runway needs to be defined as a plane. Once this equation is found, a perpendicular vector of the plane is known. Multiplying $F_{\text {Tire }}$ by the cosine of the angle between the vector perpendicular to the plane and the vector $F_{\text {Tire }}$ acts along gives the component of $F_{\text {Tire }}$ perpendicular to the surface, $F_{\text {Normal }}$. The cosine for three dimensional vectors is given by taking the dot product of the vectors and then dividing by the magnitude of the vectors. By definition, $F_{\text {Tire }}$ acts along $\overrightarrow{S D L}$. Since the magnitude of $\overrightarrow{S D L}$ is one, it can be neglected from this equation.

$$
F_{\text {Normal }}=\frac{\vec{V}_{\text {plane }} \cdot \overrightarrow{S D L}}{\left|\vec{V}_{\text {plane }}\right|} F_{\text {Tire }}
$$

Since the plane is assumed to be a horizontal plane, Equation 2.34 can be simplified further. The vector defining a horizontal plane is given by $\left[\begin{array}{lll}0 & 0 & 1\end{array}\right]_{E}^{T}$. The magnitude 
of this vector can be replaced along with its magnitude, one. When taking the dot product with one vector having only a single component, the result is that component of the vector. It follows that the cosine of the angle between the vector perpendicular to the plane representing the landing surface and $\overrightarrow{S D L}$ is simply the z-component of $[\overrightarrow{S D L}]_{E}$.

$$
F_{\text {Normal }}=S D L_{z, E} \cdot F_{\text {Tire }}
$$

With the normal force of the tire known, the maximum frictional forces in both the $X_{T}$ and the $\mathrm{Y}_{\mathrm{T}}$ directions can be found. The amount of friction for the landing gear system to apply is not always this maximum value. If the maximum value was always applied, friction would make the aircraft move when it is stationary. Maximum friction force is always applied when the tire is moving along that direction. If the aircraft is not moving in that direction however, finding the correct friction forces becomes a great deal harder. When the aircraft is stationary, all of the frictional forces together must maintain this status. To accomplish this, the frictional forces must be balanced against all of the other forces acting on the aircraft. This holds true until the point that the friction force needed to balance the other forces is greater than the maximum frictional force. When this occurs, $F_{\text {Friction,Max }}$ is used.

$$
F_{\text {Friction }}=\left\{\begin{array}{cc}
F_{\text {Friction,Max }} & \text { Velocity } \neq 0 \\
F_{\text {Balance }} & \text { Velocity }=0 \& F_{\text {Balance }} \leq F_{\text {Friction,Max }} \\
F_{\text {Friction,Max }} & \text { Velocity }=0 \& F_{\text {Balance }}>F_{\text {Friction,Max }}
\end{array}\right.
$$

Before getting to the more complicated task of determining $F_{\text {Balance }}$, the method for determining the velocity of each tire will be discussed. For the frictional force algorithm to work, the velocity of each tire must be calculated in the Body reference frame. To do this, a series of simple calculations must be made. First, the velocity of the end of each 
strut must be computed. This is found by adding the velocity components of the center of gravity relative to the Earth, $\left[\begin{array}{lll}u_{E} & v_{E} & w_{E}\end{array}\right]_{B}^{T}$, with the velocity generated by rotation about the center of gravity, $V_{r o t, B}$. $\left[\begin{array}{lll}u_{E} & v_{E} & w_{E}\end{array}\right]_{B}^{T}$ is given by subtracting the velocity of the wind from the aircraft states $\left[\begin{array}{lll}u & v & w\end{array}\right]^{T} . V_{r o t, B}$ is found by crossing the angular velocities, $\left[\begin{array}{lll}p & q & r\end{array}\right]_{B}^{T}$ with the arm connecting the center of gravity to the tire's point of contact, $\overrightarrow{C G Z}_{g}$. To compensate for the angular velocities being components, it must be represented by a square component matrix. Combining these velocities below nets the following equation:

$$
\left[V_{\text {tire }}\right]_{B}=\left[\begin{array}{c}
u_{E} \\
v_{E} \\
w_{E}
\end{array}\right]_{B}+\left[\begin{array}{ccc}
0 & -r & q \\
r & 0 & -p \\
-q & p & 0
\end{array}\right]_{B}\left[\begin{array}{l}
C G Z_{g, x} \\
C G Z_{g, y} \\
C G Z_{g, z}
\end{array}\right]_{B}
$$

The above equation gives the components of the velocity of the main landing gear. The nose landing gear needs an additional conversion after the equation is applied. Since the nose wheel can be subjected to a rotation, given by the Turning Coordinate Frame, the direction in which the tire rolls and slides must be properly adjusted. The effect of rotating the wheel causes the $\psi_{\mathrm{S}}$ value to shift accordingly. Converting the results from the Turning to the Strut Reference Frame will account for the rotation of the nose gear.

Since it is possible to cause catastrophic failures by turning too sharply at high speeds, aircraft designers limit the effectiveness of adjusting the turning angle at high velocities. The following is a model of the linear degradation of turning effectiveness between a minimum and maximum velocity threshold. From stationary to the minimum velocity, full turning effectiveness is needed. Properly modeling the deflection needs to go from 
full effectiveness to no effectiveness with no discontinuities at the minimum and maximum thresholds, respectively. After the maximum velocity is reached, the turning angle is zero. The logic for how this conversion occurs is as follows:

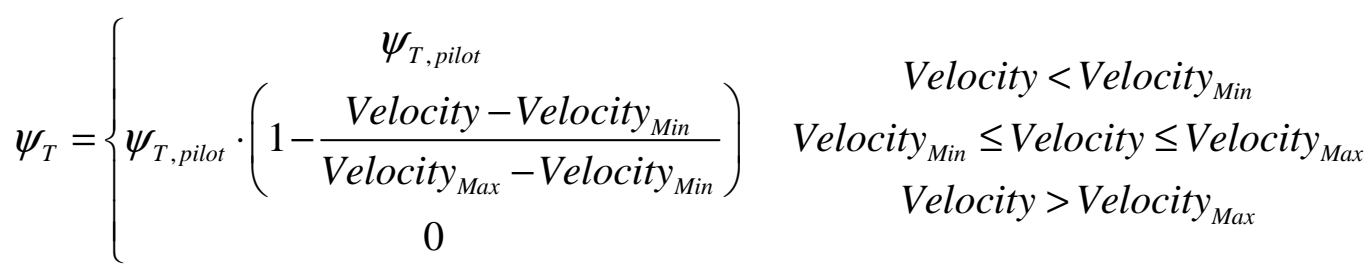

This concludes all of the calculations that need to be completed to determine the velocity of the aircraft's tires. As was stated before, when the aircraft is stationary, the friction forces must be calculated by balancing all the other forces acting on the aircraft. The friction force each strut needs to meet to hold stationary can be derived from the following set of equations where $\mathrm{F}_{\mathrm{N}}, \mathrm{F}_{\mathrm{L}}$, and $\mathrm{F}_{\mathrm{R}}$ are the forces generated in the nose, left main, and right main respectively. This gives the forces in the $\overrightarrow{O X_{E}}$ and the $\overrightarrow{O Y_{E}}$ directions. These values need to be converted to the body axis frame using the previously mentioned transformation equation. Adding this value to the previously mentioned $\overrightarrow{O Z_{S}}$ force transformed to $\overrightarrow{O Z_{B}}$ yields all of the forces generated by the landing gear.

$$
\begin{aligned}
& \vec{F}_{\text {Total }}=\vec{F}_{N}+\vec{F}_{L}+\vec{F}_{R} \\
& \vec{M}_{\text {Total }}=\overrightarrow{C G Z}_{g, N, B} \times \vec{F}_{N}+\overrightarrow{C G Z}_{g, L, B} \times \vec{F}_{L}+\overrightarrow{C G Z}_{g, R, B} \times \vec{F}_{R} \\
& \vec{F}_{L}=\vec{F}_{R}
\end{aligned}
$$

These equations are generated through balancing the net equations with the forces generated by the landing gear. The last equation comes from the aircraft being a symmetric entity. Expanding the moment equations yields the following system of equations. Additionally, the components of the position vectors are used to simplify the 
system of equation since some of the components are either zero or combine to become zero. For example the components of $\overrightarrow{C G Z}_{g, N}$ will be written as $\left[\begin{array}{lll}r_{x, N} & r_{y, N} & r_{z, N}\end{array}\right]_{B}^{T}$. To multiply these components, a square component matrix must be created as shown below.

$$
\left[\begin{array}{l}
M_{x} \\
M_{y} \\
M_{z}
\end{array}\right]_{B}=\left[\begin{array}{ccc}
0 & -r_{z, N} & r_{y, N} \\
r_{z, N} & 0 & -r_{x, N} \\
-r_{y, N} & r_{x, N} & 0
\end{array}\right]_{B}\left[\begin{array}{c}
F_{x, N} \\
F_{y, N} \\
F_{z, N}
\end{array}\right]_{B}+\left[\begin{array}{ccc}
0 & -r_{z, L} & r_{y, L} \\
r_{z, L} & 0 & -r_{x, L} \\
-r_{y, L} & r_{x, L} & 0
\end{array}\right]_{B}\left[\begin{array}{c}
F_{x, L} \\
F_{y, L} \\
F_{z, L}
\end{array}\right]_{B}+\left[\begin{array}{ccc}
0 & -r_{z, R} & r_{y, R} \\
r_{z, R} & 0 & -r_{x, R} \\
-r_{y, R} & r_{x, R} & 0
\end{array}\right]_{B}\left[\begin{array}{c}
F_{x, R} \\
F_{y, R} \\
F_{z, R}
\end{array}\right]_{B}
$$

To further reduce this moment equation, with the force in the left and the right strut being equal, both of them will be renamed $F_{M}$, yielding the following:

$$
\left[\begin{array}{l}
M_{x} \\
M_{y} \\
M_{z}
\end{array}\right]_{B}=\left[\begin{array}{ccc}
0 & -r_{z, N} & r_{y, N} \\
r_{z, N} & 0 & -r_{x, N} \\
-r_{y, N} & r_{x, N} & 0
\end{array}\right]_{B}\left[\begin{array}{l}
F_{x, N} \\
F_{y, N} \\
F_{z, N}
\end{array}\right]_{B}+\left[\begin{array}{ccc}
0 & -\left(r_{z, L}+r_{z, R}\right) & \left(r_{y, L}+r_{y, R}\right) \\
\left(r_{z, L}+r_{z, R}\right) & 0 & -\left(r_{x, L}+r_{x, R}\right) \\
-\left(r_{y, L}+r_{y, R}\right) & \left(r_{x, L}+r_{x, R}\right) & 0
\end{array}\right]_{B}\left[\begin{array}{l}
F_{x, M} \\
F_{y, M} \\
F_{z, M}
\end{array}\right]_{B}
$$

For this model of the aircraft, as is true with most aircrafts, $r_{y, N}=0, r_{y, L}=r_{y, R}=r_{y, M}$, and $r_{y, L}=-r_{y, R}$. This yields the following equations when combined with the force equations:

$$
\begin{aligned}
& {\left[\begin{array}{l}
F_{x} \\
F_{y} \\
F_{z}
\end{array}\right]_{B}=\left[\begin{array}{lll}
1 & 0 & 0 \\
0 & 1 & 0 \\
0 & 0 & 1
\end{array}\right]\left[\begin{array}{l}
F_{x, N} \\
F_{y, N} \\
F_{z, N}
\end{array}\right]_{B}+\left[\begin{array}{lll}
2 & 0 & 0 \\
0 & 2 & 0 \\
0 & 0 & 2
\end{array}\right]\left[\begin{array}{l}
F_{x, M} \\
F_{y, M} \\
F_{z, M}
\end{array}\right]_{B}} \\
& {\left[\begin{array}{l}
M_{x} \\
M_{y} \\
M_{z}
\end{array}\right]_{B}=\left[\begin{array}{ccc}
0 & -r_{z, N} & 0 \\
r_{z, N} & 0 & -r_{x, N} \\
0 & r_{x, N} & 0
\end{array}\right]_{B}\left[\begin{array}{l}
F_{x, N} \\
F_{y, N} \\
F_{z, N}
\end{array}\right]_{B}\left[\begin{array}{ccc}
0 & -\left(r_{z, L}+r_{z, R}\right) & 0 \\
\left(r_{z, L}+r_{z, R}\right) & 0 & -2 r_{x, M} \\
0 & 2 r_{x, M} & 0
\end{array}\right]_{B}\left[\begin{array}{l}
F_{x, M} \\
F_{y, M} \\
F_{z, M}
\end{array}\right]_{B}}
\end{aligned}
$$

These equations can be further reduced into a single matrix equation as is given below. 


$$
\left[\begin{array}{c}
F_{x} \\
F_{y} \\
F_{z} \\
M_{x} \\
M_{y} \\
M_{z}
\end{array}\right]_{B}\left[\begin{array}{cccccc}
1 & 0 & 0 & 2 & 0 & 0 \\
0 & 1 & 0 & 0 & 2 & 0 \\
0 & 0 & 1 & 0 & 0 & 2 \\
0 & -r_{z, N} & 0 & 0 & -\left(r_{z, L}+r_{z, R}\right) & 0 \\
r_{z, N} & 0 & -r_{x, N} & \left(r_{z, L}+r_{z, R}\right) & 0 & -2 r_{x, M} \\
0 & r_{x, N} & 0 & 0 & 2 r_{x, M} & 0
\end{array}\right]_{B}\left[\begin{array}{l}
F_{x, N} \\
F_{y, N} \\
F_{z, N} \\
F_{x, M} \\
F_{y, M} \\
F_{z, M}
\end{array}\right]_{B}
$$

Further examination into this system of equations shows that there are in reality two systems of equations as shown below. One of the systems is an independent system with an irrelevant third equation. The other system is an indeterminant system of equations with four variables in three equations. From the latter, the forces in the y-direction can be calculated. The force in the $\mathrm{x}$-direction must be derived by multiplying the $\mathrm{x}$-component of $F_{\text {Total }}$ by the load percentage at steady state. This is only accurate when the aircraft is stationary.

$$
\begin{aligned}
& {\left[\begin{array}{c}
F_{y} \\
M_{x} \\
M_{z}
\end{array}\right]_{B}=\left[\begin{array}{cc}
1 & 2 \\
-r_{z, N} & -\left(r_{z, L}+r_{z, R}\right) \\
r_{x, N} & 2 r_{x, M}
\end{array}\right]_{B}\left[\begin{array}{c}
F_{y, N} \\
F_{y, M}
\end{array}\right]_{B}} \\
& {\left[\begin{array}{c}
F_{x} \\
F_{z} \\
M_{y}
\end{array}\right]_{B}=\left[\begin{array}{cccc}
1 & 0 & 2 & 0 \\
0 & 1 & 0 & 2 \\
r_{z, N} & -r_{x, N} & \left(r_{z, L}+r_{z, R}\right) & -2 r_{x, M}
\end{array}\right]_{B}\left[\begin{array}{c}
F_{x, N} \\
F_{z, N} \\
F_{x, M} \\
F_{z, M}
\end{array}\right]_{B}}
\end{aligned}
$$

\section{E. Physical Limitations}

So far, the modeling of the landing gear system has not considered physical limitations due to the fact that components of the system are rigid solid bodies that cannot penetrate each other. These limitations need to be placed on how far the points on the SDL can move in relation to the strut. The first case is that the distance from the top of 
the strut to the ground can not be less than the distance between the top of the strut and the hub of the wheel. The second limitation stops the wheel hub from entering the fuselage of the airplane. Lastly, the third limitation is created by combining the prior two. For modeling purposes, the violation of these limitations can be interpreted as due to insufficient force produced by the various system components.

\section{E1. Insufficient Tire Force}

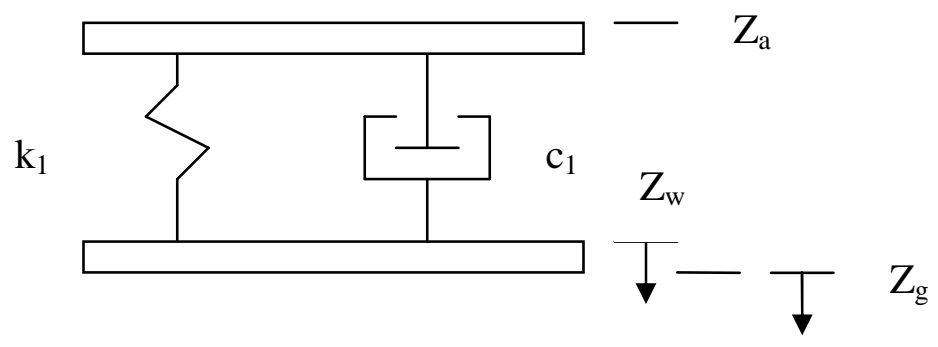

Figure 9. Dynamic model of a landing gear with insufficient tire force

This scenario occurs if there is insufficient force generated by the tire to keep the hub of the wheel from coming into contact with the ground. Low tire pressure, loss of tire, or excessively hard landings could cause this event to happen. Since the force of the tire is no longer part of the dynamics of the system, the dynamic equations must be changed to reflect this. First off, this situation only occurs if $\left|\overrightarrow{Z_{a} Z_{g}}\right| \leq\left|\overrightarrow{Z_{a} Z_{w}}\right|$. This condition invokes a change to $\left|\overrightarrow{Z_{a} Z_{w}}\right|$ and $\left(\left|\overrightarrow{Z_{a} Z_{w}}\right|\right)_{S}$. In this case, $\left(\left|\overrightarrow{Z_{a} Z_{w}}\right|\right)_{S}$ is found by taking the numerical derivative between $\left|\overrightarrow{Z_{a} Z_{w}}\right|$ generated the time step prior to this condition and $\left|\overrightarrow{Z_{a} Z_{g}}\right|$. This change affects the equations for $F_{\text {oleo }}$ and $F_{\text {tire }} \cdot F_{\text {oleo }}$ is basically the same equation as before but $\left|\overrightarrow{Z_{a} Z_{g}}\right|$ is used in place of $\left|\overrightarrow{Z_{a} Z_{w}}\right|$. Since $F_{\text {tire }}$ is 
used in determining the frictional force against the landing surface, the value is found by setting the acceleration of the wheel hub to zero. To determine if this case ends, the integration needs to take in account $F_{\text {tire,orignal }}$ instead of $F_{\text {tire }}$ calculated in this manner. Otherwise, the wheel hub would never move.

$$
\begin{aligned}
& \left|\overrightarrow{Z_{a} Z_{w}}\right|=\left|\overrightarrow{Z_{a} Z_{g}}\right| \text { if }\left|\overrightarrow{Z_{a} Z_{g}}\right| \leq\left|\overrightarrow{Z_{a} Z_{w}}\right| \\
& \left(\left|\overrightarrow{Z_{a} Z_{w}}\right|\right)_{S}=\left(\left|\overrightarrow{Z_{a} Z_{g}}\right|\right)_{S} \text { if }\left|\overrightarrow{Z_{a} Z_{g}}\right| \leq\left|\overrightarrow{Z_{a} Z_{w}}\right| \\
& F_{\text {oleo }}=-k_{1}\left(\left|\overrightarrow{Z_{a} Z_{g}}\right|-\left|\overrightarrow{Z_{a} Z_{w, \text { unloaded }}}\right|\right)-c_{1}\left(\left|\overrightarrow{Z_{a} Z_{g}}\right|\right)_{S} \\
& F_{\text {tire }}=F_{\text {oleo }}+m_{2} g_{z, S} \\
& \left(\left|\overrightarrow{Z_{a} Z_{w}}\right|\right)_{S}=\int\left[\frac{1}{m_{2}} F_{\text {oleo }}-\frac{1}{m_{2}} F_{\text {tire,orignal }}+g_{z, S}\right] \\
& \left|\overrightarrow{Z_{a} Z_{w}}\right|=\int\left(\left|\overrightarrow{Z_{a} Z_{w}}\right|\right)_{S}
\end{aligned}
$$

\section{E2. Insufficient Oleo Force}

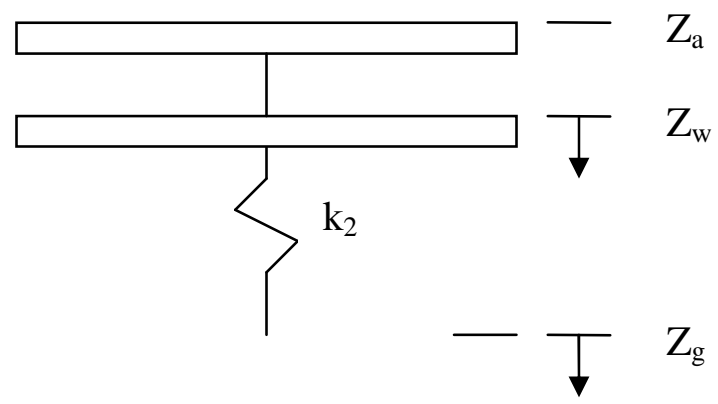

Figure 10. Dynamic model of a landing gear with insufficient oleo force

This scenario occurs if there is insufficient force generated by the oleo to keep the hub of the wheel from coming into contact with the body of the aircraft. There is a 
minimum distance that the wheel can be away from $Z_{a}$. This is denoted as $\left|\overrightarrow{Z_{a} Z_{w_{\text {min }}}}\right|$. This minimum distance could be created because the wheel comes into contact with the body of the aircraft or the oleo can only depress to a certain degree. Loss of hydraulic pressure or excessively hard landings could cause this event to happen. Since the force of the oleo is no longer part of the dynamics of the system, the dynamic equations must be changed to reflect this. First off, this situation only occurs if $\left|\overrightarrow{Z_{a} Z_{w}}\right| \leq\left|\overrightarrow{Z_{a} Z_{w}}{ }_{\text {min }}\right|$. This alteration causes a change to the equations for $F_{\text {oleo }} . F_{\text {tire }}$ is the same equation as before with the exception $\left|\overrightarrow{Z_{a} Z_{w}}\right|$ is constrained to being $\left|\overrightarrow{Z_{a} Z_{w}}\right|$. Balancing the forces on the wheel hub give the equation for $F_{\text {oleo }}$. To determine if this case ends, the integration needs to take in account $F_{\text {oleo,orignal }}$ instead of $F_{\text {oleo }}$ calculated in this manner. Otherwise, the wheel hub would never move.

$$
\begin{aligned}
& \left|\overrightarrow{Z_{a} Z_{w}}\right|=\left|\overrightarrow{Z_{a} Z_{w_{\text {min }}}}\right| \quad \text { if }\left|\overrightarrow{Z_{a} Z_{w}}\right| \leq\left|\overrightarrow{Z_{a} Z_{w_{\min }}}\right| \\
& F_{\text {oleo }}=F_{\text {tire }}-m_{2} g_{z, S} \\
& F_{\text {tire }}=k_{2}\left(\operatorname{Rad}_{\text {tire }}-\left(\left|\overrightarrow{Z_{a} Z_{g}}\right|-\left|\overrightarrow{Z_{a} Z_{w_{\text {min }}}}\right|\right)\right) \\
& \left(\left|\overrightarrow{Z_{a} Z_{w}}\right|\right)_{S}=\int\left[\frac{1}{m_{2}} F_{\text {oleo, orignal }}-\frac{1}{m_{2}} F_{\text {tire }}+g_{z, S}\right] \\
& \left|\overrightarrow{Z_{a} Z_{w}}\right|=\int\left(\left|\overrightarrow{Z_{a} Z_{w}}\right|\right)_{S}
\end{aligned}
$$




\section{E3. Insufficient Tire and Oleo Force}

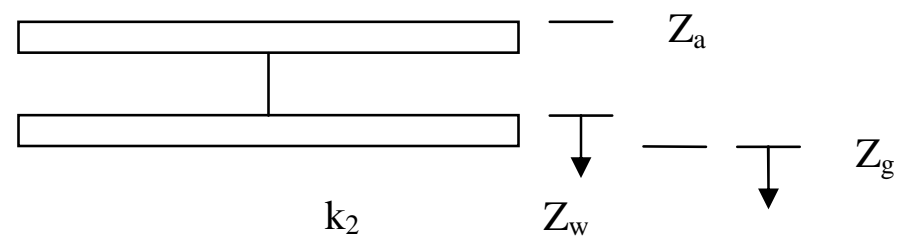

Figure 11. Dynamic model of a landing gear with insufficient tire and oleo force

This scenario occurs if the other two cases of sub-sections E1 and E2 occur simultaneously. For this situation, the only force created is that by the reaction of the landing surface. This reaction passes directly from the landing surface, through the tire, to the oleo. This makes the $F_{\text {oleo }}$ being equal to the $F_{\text {tire }}$.

$$
\begin{aligned}
& F_{\text {oleo }}=F_{\text {tire }}=F_{\text {LandingSurface }} \\
& \left(\left|\overrightarrow{Z_{a} Z_{w}}\right|\right)_{S}=\int\left[\frac{1}{m_{2}} F_{\text {oleo,orignal }}-\frac{1}{m_{2}} F_{\text {tire, orignal }}+g_{z, S}\right] \\
& \left|\overrightarrow{Z_{a} Z_{w}}\right|=\int\left(\left|\overrightarrow{Z_{a} Z_{w}}\right|\right)_{S}
\end{aligned}
$$




\section{III.Failure Modeling}

Landing gear failures can be separated into one of two types: Component Degradation and Jamming. Some of these effects are only applicable to certain landing struts.

\section{A. Component Degradation}

Component Degradation occurs when a component of the dynamic model is subjected to the effects of aging and insecure mechanical connections. This can be due to one of the parts of the system(s) being submitted to fatigue, excessive loading, or aircraft vibrations loosening components of the landing gear. (i.e. old aircrafts, extremely hard landings, or bolts not being tightened). Considering the three component model as depicted above (Fig. 2), there are three types of dynamic degradation failures modeled within this effort: Oleo Spring Degradation, Oleo Damper Degradation, and Tire Degradation. A fourth type of failure comes in the form of Brake Degradation.

\section{A1. Oleo Spring Degradation}

In this case, the spring component of the oleo becomes altered from the normal system operating conditions. This event can take place on any of the struts. A failure of this type is most prevalent upon coming in contact with the landing surface with extreme contact force. To model this effect, the spring constant value, $\mathrm{K}_{1}$, is multiplied by a user submitted multiplier $\mu_{\text {altered }}$ at all moments in time after the time of the failure, $t_{f}$. If the spring becomes stiffer, the multiplier will be greater than 1 . Weakening of the spring yields a multiplier between 0 and 1 . 


$$
K_{1}(t)= \begin{cases}K_{1, \text { orig }} & \text { for } t<t_{f} \\ K_{1, \text { orig }} * \mu_{\text {altered }} & \text { for } t \geq t_{f}\end{cases}
$$

\section{A2. Oleo Damper Degradation}

For this failure to occur, the damping power of the oleo is altered. This could happen by the hydraulic fluid of some systems to be reduced, by perhaps a leaky containment unit. Once again, this type of failure is applicable to any of the three landing gear struts. Multiplying the $\mathrm{C}_{1}$ value by an alteration factor after the failure occurs is an appropriate representation of this event. Corrosion of the damper system would raise the damper coefficient, whereas a loss in hydraulic fluid would lower this value. A touch of caution needs to be employed when raising the damper coefficient as this could cause the dynamic model to require smaller integration steps.

$$
C_{1}(t)= \begin{cases}C_{1, \text { orig }} & \text { for } t<t_{f} \\ C_{1, \text { orig }} * \mu_{\text {altered }} & \text { for } t \geq t_{f}\end{cases}
$$

\section{A3. Tire Degradation}

This event occurs when the tire is no longer kept at the designed system pressures.

Excessive air in the tire would raise the spring coefficient. Lacking air pressure lowers the spring coefficient until the point the spring coefficient becomes zero. This zero spring coefficient is actually a common occurrence. That would occur if a complete blow out of the tire would occur or the wheel fell from the aircraft. From a dynamic modeling perspective along the SDL, either cause of wheel loss behaves similarly. Modeling this effect is accomplished by adjusting the $\mathrm{K}_{2}$ value to a new value after the failure occurs. 


$$
K_{2}(t)= \begin{cases}K_{2, \text { orig }} & \text { for } t<t_{f} \\ K_{2, \text { orig }} * \mu_{\text {altered }} & \text { for } t \geq t_{f}\end{cases}
$$

Depending on how the wheel explodes in the case of a blowout, two different effects can occur. If the exhaust from the explosion is expelled along the rolling surface, in the plane created by $\mathrm{X}_{\mathrm{B}}$ and $\mathrm{Z}_{\mathrm{B}}$, additional effects are not considered. The logic behind this is twofold. First, if the plane is in motion then the tire will be rotating. This will cause the place of exhaust to rotate also, dissipating the effect. Secondly and more importantly, most aircrafts have a considerable amount of weight compared to the amount of force an exploding tire would create. A lot more force would be needed to affect the system. The other case is if the exhaust comes from the side of the tire. In the case of the guiding landing gear, usually the nose, the wheel can rotate about the strut. In the case of the mains, rotation is considered restrained for this model. Modeling of this failure is quite difficult due to the unknown degree of deflection. Tire size, mass of guiding strut, distance of exhaust from axis, and load on tire are just a few examples of variables that can affect the correct amount of twisting to apply to the nose wheel. To appease this conundrum, the degree of twisting is left variable to the user $\psi_{T, \text { failure }}$. Modeling this occurs by adding an additional spike in the turning angle, $\psi_{\mathrm{T}}$, and applying a torque to the joystick. Once again, this is only applicable on the nose wheel.

$$
\psi_{T}(t)= \begin{cases}\psi_{T, \text { Pilot }} & \text { for } t<t_{f} \\ \psi_{T, \text { Pilot }}+\psi_{T, \text { Failure }} & \text { for } t=t_{f} \\ \psi_{T, \text { Pilot }} & \text { for } t>t_{f}\end{cases}
$$




\section{A4. Brake Degradation}

The effect of altering the braking system is negligible when viewing the system dynamics along the strut deflection line. It does alter other components of the landing gears' generated forces, namely the maximum frictional force in the direction of motion. If something becomes lodged in the braking apparatus the degree of friction between the rotors and the brake pads would be increased. Additionally, as the brakes are used, the braking capabilities of the aircraft will be reduced. With brakes, the maximum frictional force value is based only on the interaction between $\mathrm{F}_{\text {Normal }}$ and $\mu_{\text {friction. }}$. Since there are no brakes on the nose wheel of most aircrafts, this failure is restricted to only the main landing gear.

$$
\mu_{\text {Brake }}(t)= \begin{cases}\mu_{\text {Brake, pilot }} & \text { for } t<t_{f} \\ \mu_{\text {Brake, pilot }} * \mu_{\text {altered }} & \text { for } t \geq t_{f}\end{cases}
$$

\section{A5. Shimmy}

Shimmy is usually an oscillating torsion of the steering wheel caused by vibrations of the aircraft. This event occurs outside of the pilot's control. Vibrations, wearing of the landing strut, and poor runway conditions are claimed to be the cause of this effect. With this thought in hand, modeling this failure is done by injecting a vibration to the Strut Reference Angles. With different flight parameters, the vibrations can have a range of frequencies and amplitudes. To allow this capability, the values of these parameters $\left(\psi_{S, \text { user }}\right.$ and $\left.\omega_{\psi, \text { user }}\right)$ for this modeling are left variable to be input by the user. Since the effect does not occur at a specific time but usually occurs over a period of time, $t_{\mathrm{f}}$ for this failure will be $t_{0}$ often. For the most part, this effect is seen in the variations of the turning angle depicted as $\psi$ but it can occur in the other two angles that are not modeled. 


$$
\psi_{S}(t)=\psi_{\mathrm{S}}+\psi_{\mathrm{S} \text {,user }} \cos \left(\omega_{\psi, \text { user }} \mathrm{t}\right) \text { for } t \geq t_{f}
$$

\section{B. Jamming}

Jamming occurs when a component of the aircraft is stuck in one position. Examples of how this can occur are when debris becomes wedged in the workings of the aircraft or poor lubrication. Two devices are prone to this type of event, the oleo and the brakes.

\section{B1. Oleo Jamming}

A jammed oleo means that the distance between $Z_{a}$ and $Z_{w}$ is constant. This greatly affects how the dynamics of the system act. The oleo begins to act as if it were a rigid body. With Newton's Third Law in mind, a rigid oleo would dictate that the force in the tire would be transmitted directly through the strut, ergo $F_{\text {strut }}=F_{\text {Tire. }}$ Failure of this type can occur on any of the three landing struts.

$$
F_{\text {oleo }}(t)= \begin{cases}-k_{1}\left(\left|\overrightarrow{Z_{a} Z_{w}}\right|-\left|\overrightarrow{Z_{a} Z_{w}, \text { unloaded }}\right|\right)-c_{1}\left|\overrightarrow{Z_{a} Z_{w, s}}\right| & \text { for } t<t_{f} \\ F_{\text {Tire }}-m_{2} g_{z, S} & \text { for } t \geq t_{f}\end{cases}
$$

\section{B2. Brake Jamming}

Jammed brakes apply a constant deceleration of forward motion. The amount of deceleration that is created is variable based on how much braking pressure is being applied at the time of the jam. If full pressure is being applied, then the entire braking force would be generated whereas a slight braking pressure would generate only a slight slowing effect. Compare slamming on the brakes of a vehicle to stop from hitting 
someone crossing the road with leisurely coming to a stop at a well visible red light. Thinking of this, a variable braking force must be implemented for an accurate model. When using a flight simulator, the best way to handle this circumstance is to have the braking force user-defined so as to allow for all possible degrees of failure. Since this is a braking issue, this can only occur on the main struts.

$$
\mu_{\text {Brake }}(t)= \begin{cases}\mu_{\text {Brake, pilot }} & \text { for } t<t_{f} \\ \mu_{\text {Brake, failure }} & \text { for } t \geq t_{f}\end{cases}
$$




\section{Simulink Implementation}

The simulation environment consists of three main modules as illustrated in Figure 12: input, output, and simulation nucleus. The Input Module drives the Simulation Nucleus. From this module, inputs to the simulation system are generated. The input can be from a pilot for training capabilities or pre-recorded to derive more accurate comparable results for landing system testing. Simulation Parameters determine the failure types along with other testing characteristics such as wind.

The Simulation Nucleus consists of the following sub-modules:

1. Aerodynamics Model. It consists of the characteristic equations related to this specific aircraft. Inputs are the deflection of the control surfaces and the states of the aircraft. Output is aerodynamic forces and moments.

2. Engine Model. This module models the jet engine for this craft. The input is the states of the aircraft and the degree of throttle given by the pilot. Output is propulsion force and moments.

3. Sum of Forces and Moments / Equations of Motion. This module performs the collection of all forces and moments.

4. Equations of Motion. This module includes the calculation of the states of the aircraft. This is done by combining the previous states with force and moment equations and integrating.

5. Landing System Model. In this block the dynamic modeling of the landing system takes place with adjustments given by the Failure Modeling module. For this module to work, the states of the aircraft, braking parameters given by the pilot, 
and additional forces and moments produced must be known. The Simulink® modeling of this section will be discussed in the following subsection.

6. Failure Model. In this module, the specified failures are condensed and issued to be employeed by the Landing System Model. The Simulink® modeling of this section will be discussed in the subsection following the Landing System Model.

The Output Module expresses the results generated by the simulation and provides the output interface to the user. It is divided into 3 different sub-modules. A visualization environment exists when used in conjunction with X-Plane®. A set of output data selected by the user is saved to the computer disc and time histories of relevant parameters may be monitored during the simulation or generated after.

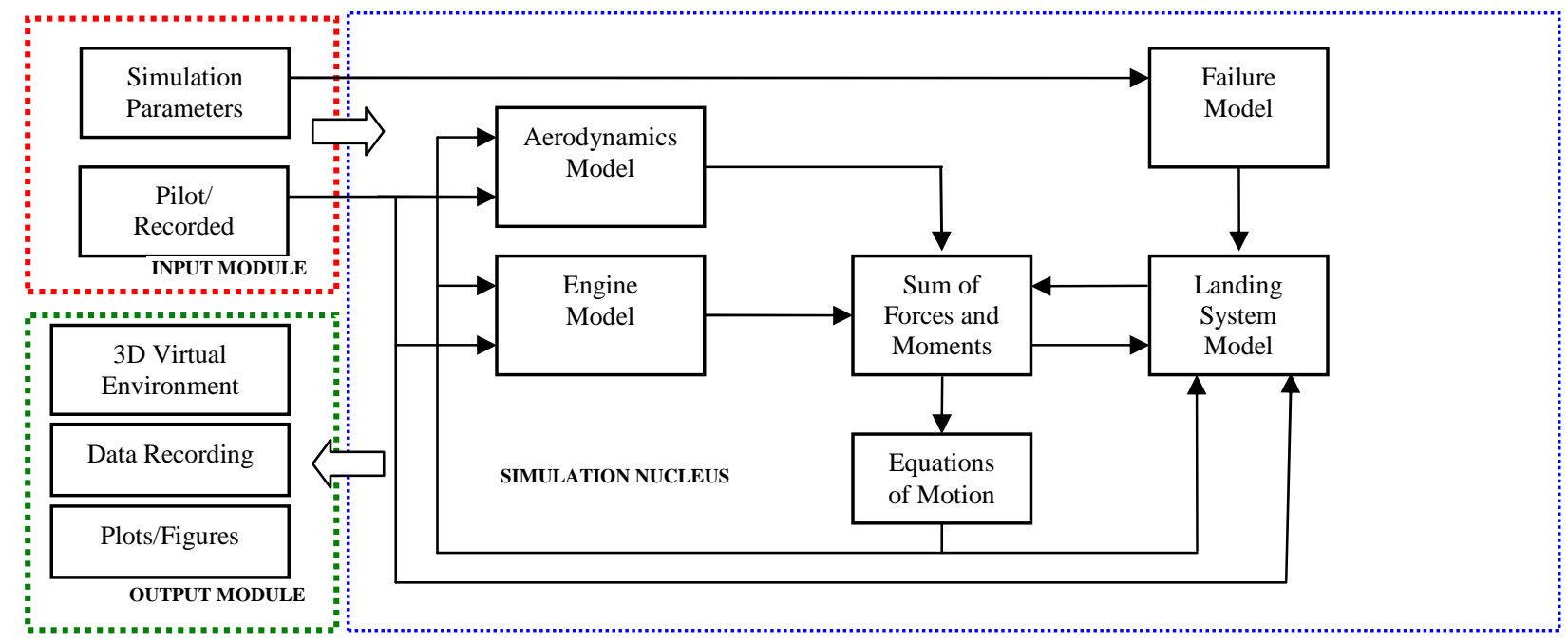

Figure 12. General diagram of the different modules of the simulation environment

The Simulink® block responsible for modeling the landing gear system can be seen below. It is within this block that all of the calculations dealing with the landing gear model take place. As can be seen, there are seven inputs and three outputs. The uvw input includes the components in Body axes of the aircraft velocity relative to the 
Earth. $\mathrm{x}$ represents the set of all the state variables of the airplane model mentioned above. Force Tot and Moment Tot are the total sums of the forces and moments acting on the aircraft. The brake command, steering, and deployment are all pilot generated inputs. Outputs of this block are the forces and moments generated by the landing gear. Additionally a notice if the wheels are on the ground is also outputted by the block.

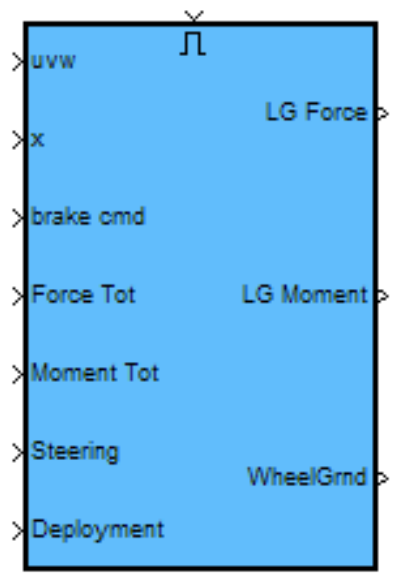

Figure 13. Highest level of landing gear modeling block.

After opening the block, Figure 14 is seen without the block segmented into phases of the calculation. In Area $1,\left[\overrightarrow{O_{E} Z_{a}}\right]_{E}$ for each landing gear is calculated along with the equation for the landing surface. Area 2 calculates $\left|\overrightarrow{Z_{a} Z_{g}}\right|$ relative to a horizontally smooth landing surface. The third area derives the force each strut produces. Frictional forces are calculated in Area 4. The total combined force of the landing gear and the combined moments they create are what is done with Area 5 of the block. The sixth area determines if the failure situation should be triggered and how long the effect of a blow out occurs. The last area, Area 7, is an additional check to see if any nonlanding gear parts of the aircraft come into contact with the landing surface. 


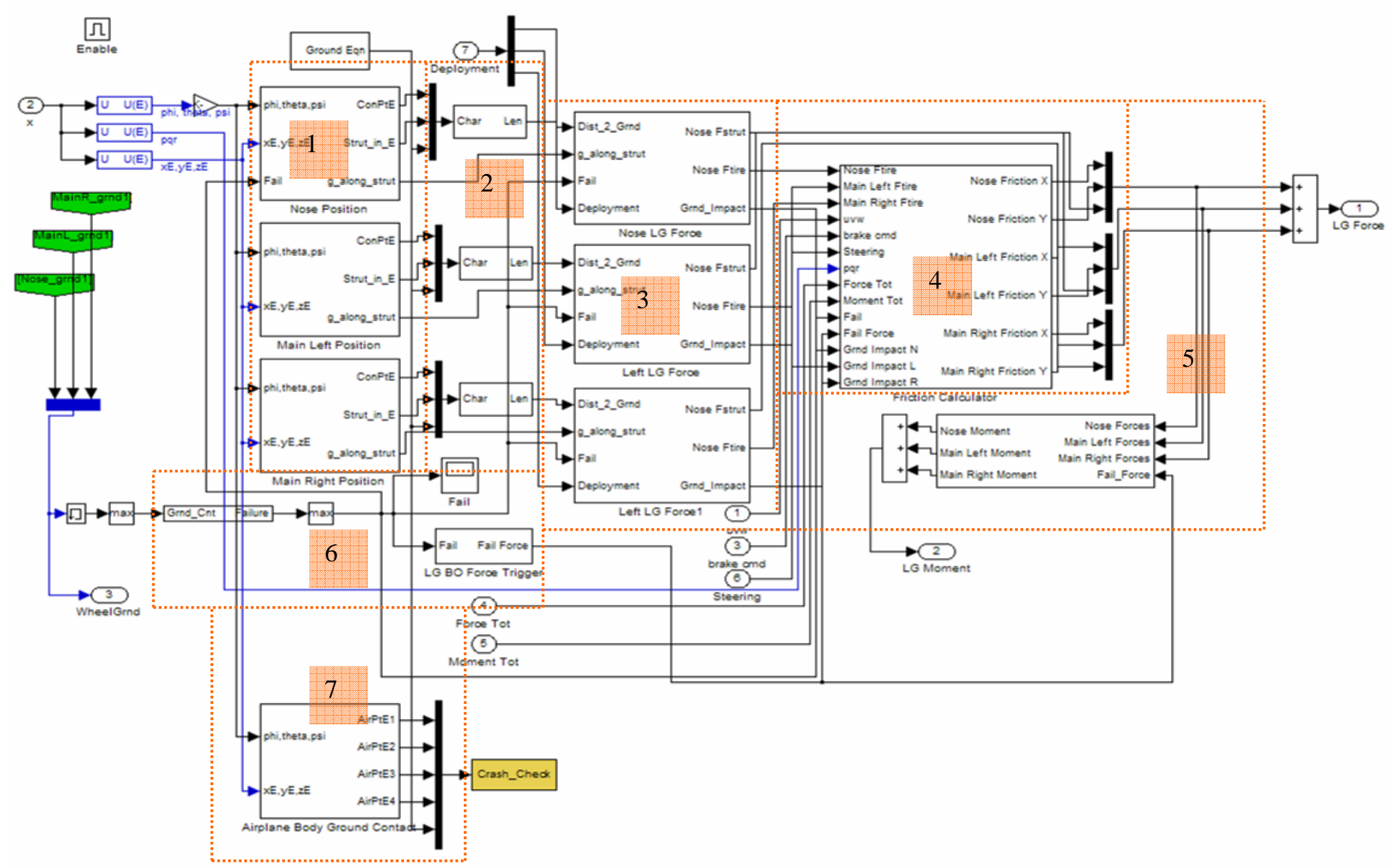

Figure 14. View of the landing gear modeling stages. 


\section{Area 1 Discussion:}

Looking under the nose position finding subsystem block in the first area opens up the following figure. ConPtE outputs $\left[\overrightarrow{O_{E} Z_{a}}\right]_{E}$. Strut_in_E is the SDL unit components in the Earth reference frame. At this point, the component of gravitational acceleration along the strut is calculated to be used in the dynamic response equations. This is the output given as g_along_strut. At the bottom of the model the injection method for shimmy can be seen. As can be seen from the trigonometric sine block, shimmy is modeled as an additional oscillation of the turning angle.

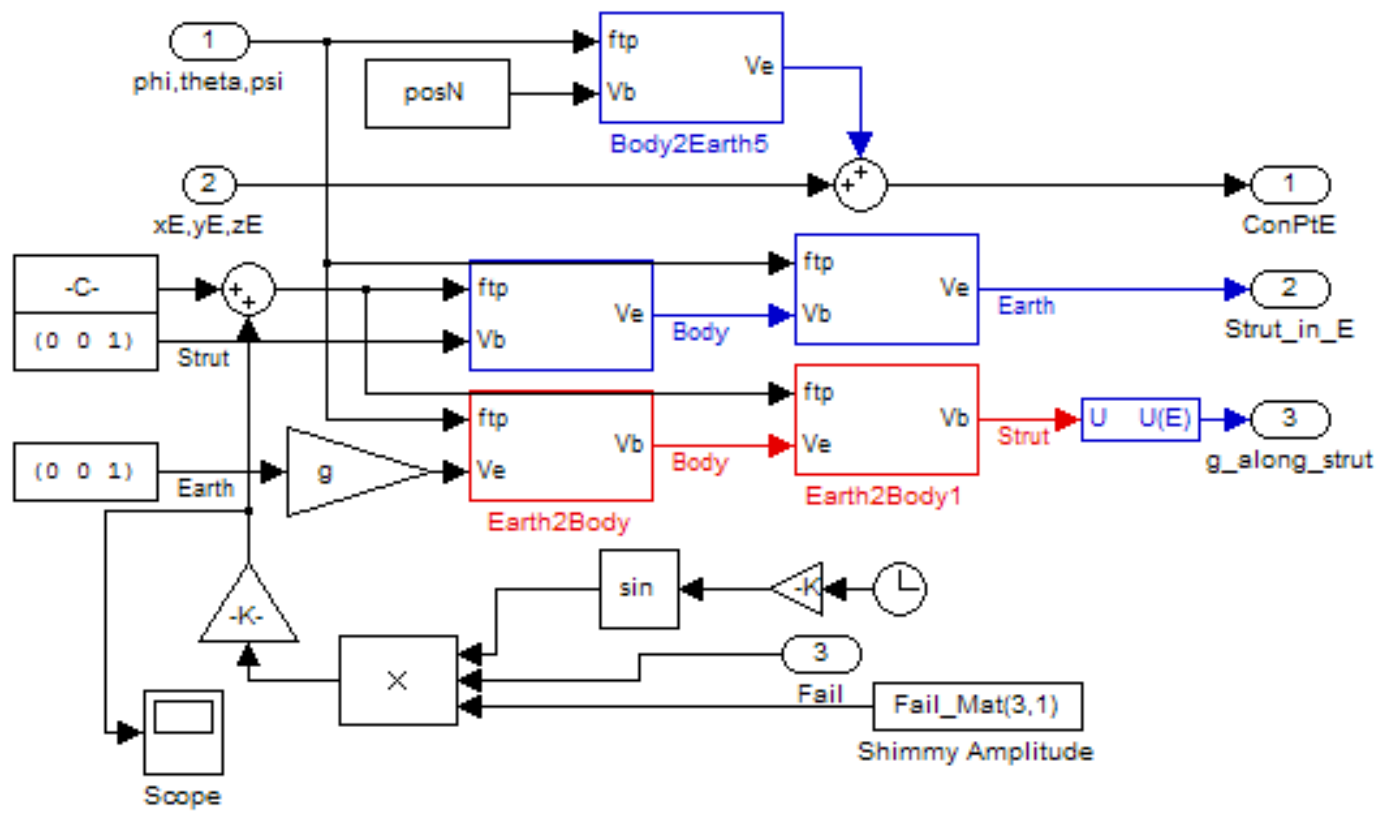

Figure 15. Calculating the strut length.

Area 2 Discussion:

The second area is simply the implementation of Equation 2.22.

Area 3 Discussion:

In the third area, the forces generated by each of the landing gears are calculated. This is done by having different modes for different types of failures as shown below in 
Figure 16 as subsection 2. Since the dynamics of the each system is different, the position of $Z_{\mathrm{w}}$ must be passed among the subsections as they change. Which mode that is to be used in the calculations is chosen by a routing system based off of selected failures in the first and third subsections. The fourth subsection is where it is determined if the pilot generated failures pass the failure threshold.

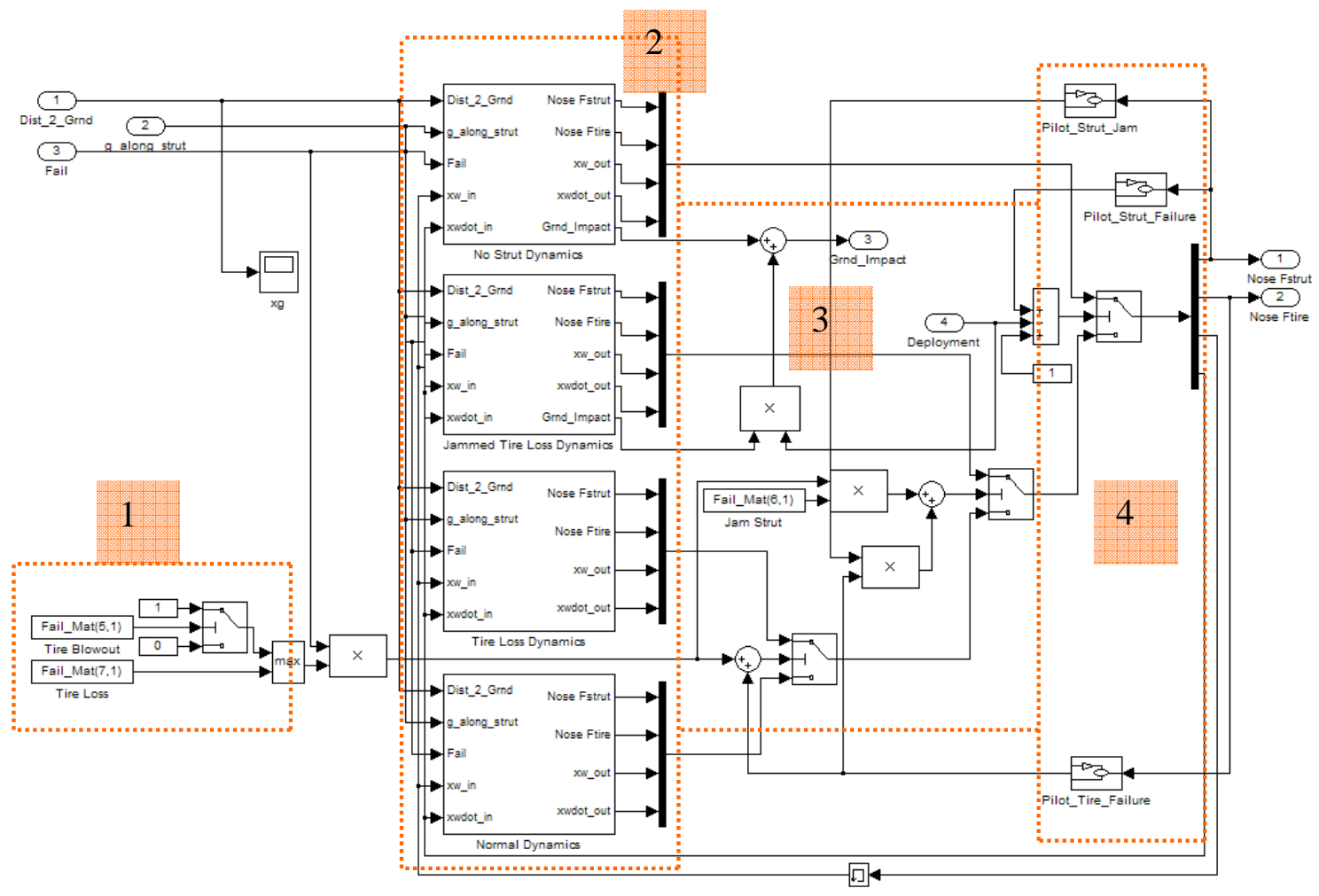

Figure 16. View of different landing gear modes.

Examining the normal dynamic mode of the second subsystem yields the following figure, Figure 17. The first subsection is where the physical limitations are placed on the model. Subsection 2 is where the integrals are computed. In Subsections 3 and 4, the tire and oleo forces are calculated respectively. Failure variables are determined in the last subsection. 


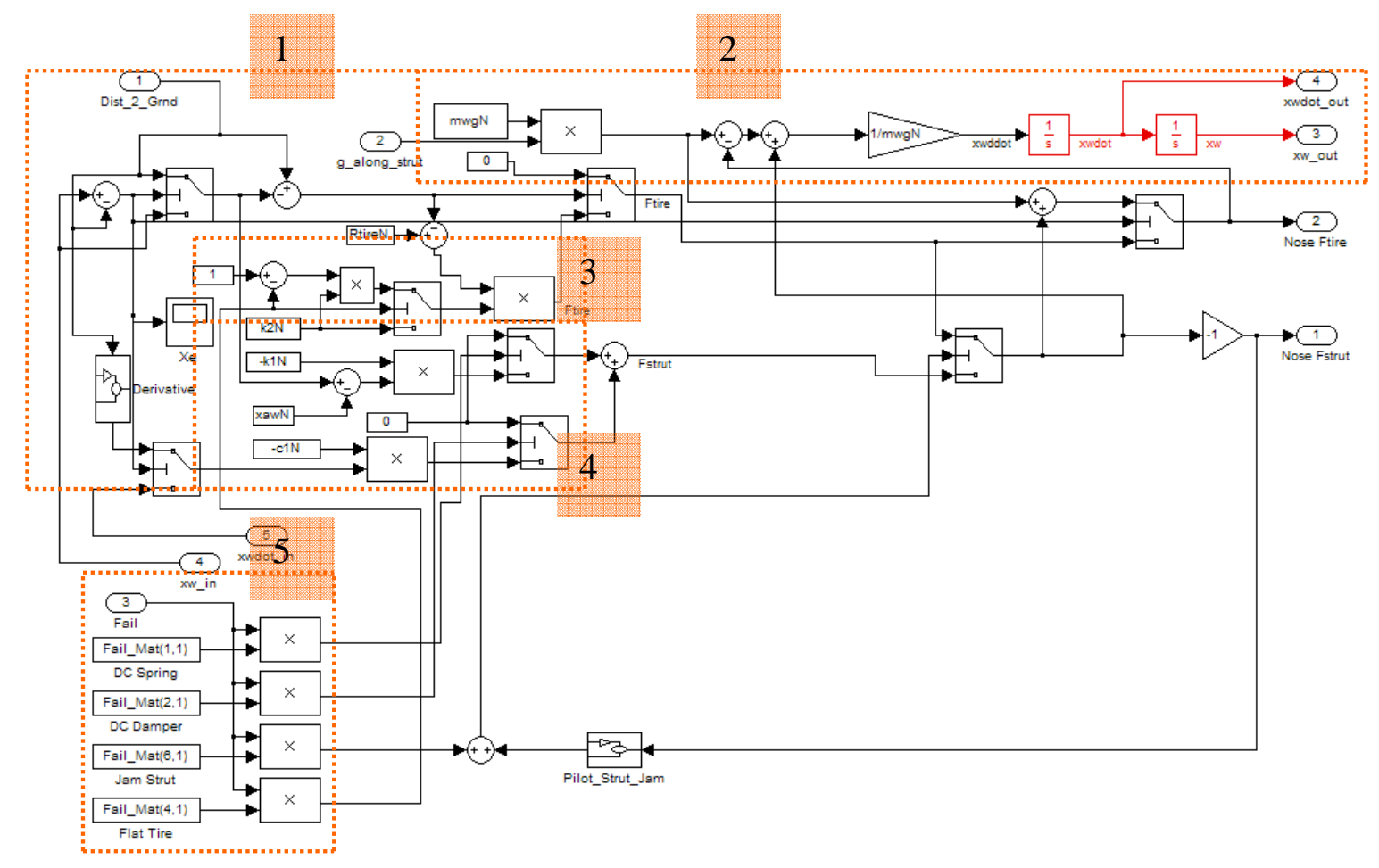

Figure 17. Normal dynamic model of landing gear forces.

Area 4 Discussion:

From Figure 18 it can be seen that Area 4 block can be divided into two parts. In the first subsection the maximum possible friction force in the $O_{T} X_{T}$ and $O_{T} Y_{T}$ directions and the velocity of the tire along these axes. The maximum friction takes into account failures that cause alteration of the coefficient of friction. Calculation of velocity is performed according to equation (2.27). The turning angle is added to the nose wheel calculations. For the rest of the struts, there is no additional angle since it is assumed that these wheels are incapable of rotation. These calculations can be seen in Figure 19. Figure 20 deals with routing the frictional forces into the proper blocks. Balancing of the motion equations can be seen in the center of this block. Figure 21 shows how the friction algorithm is applied. 


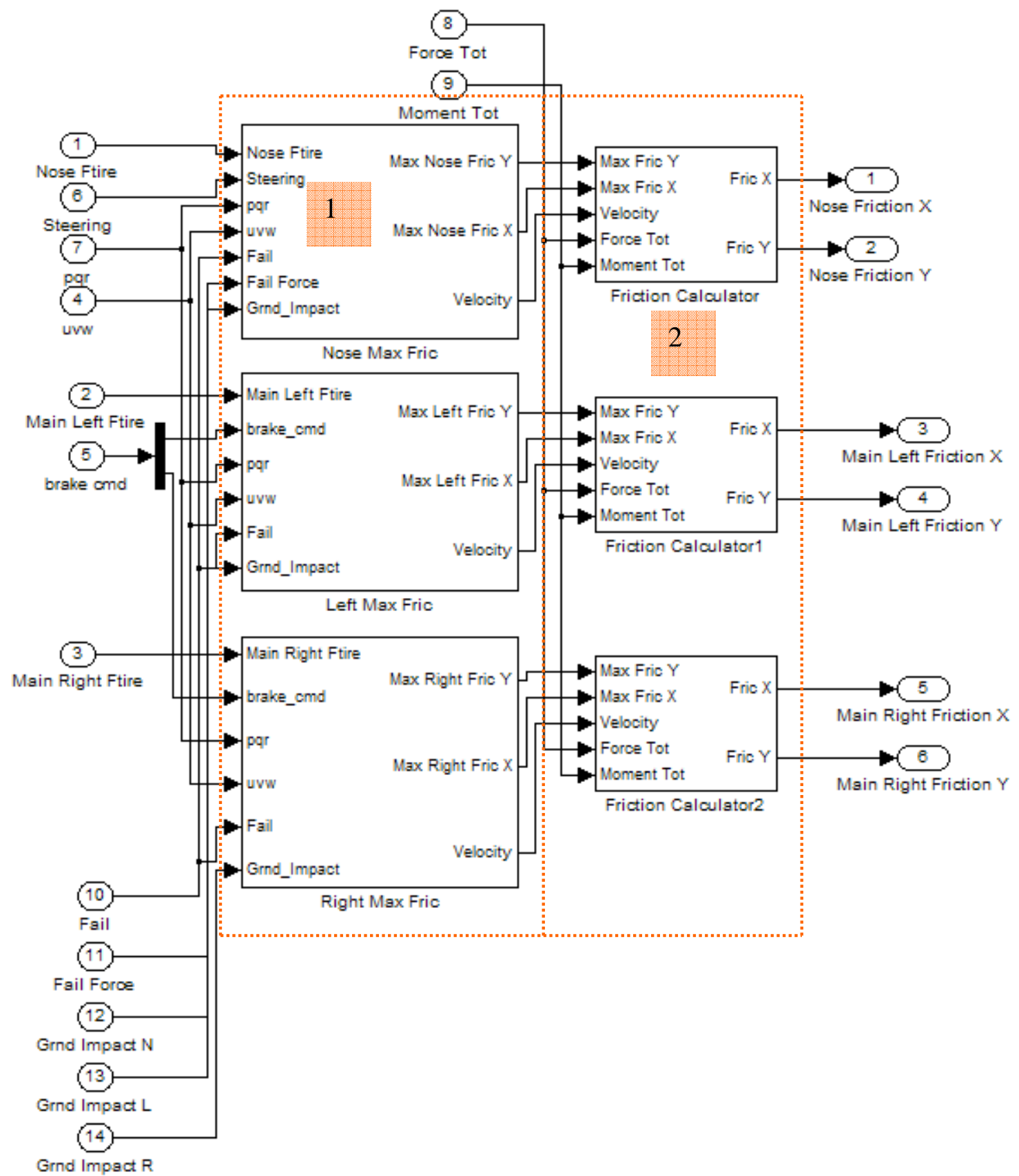

Figure 18. Internal components of friction calculator. 


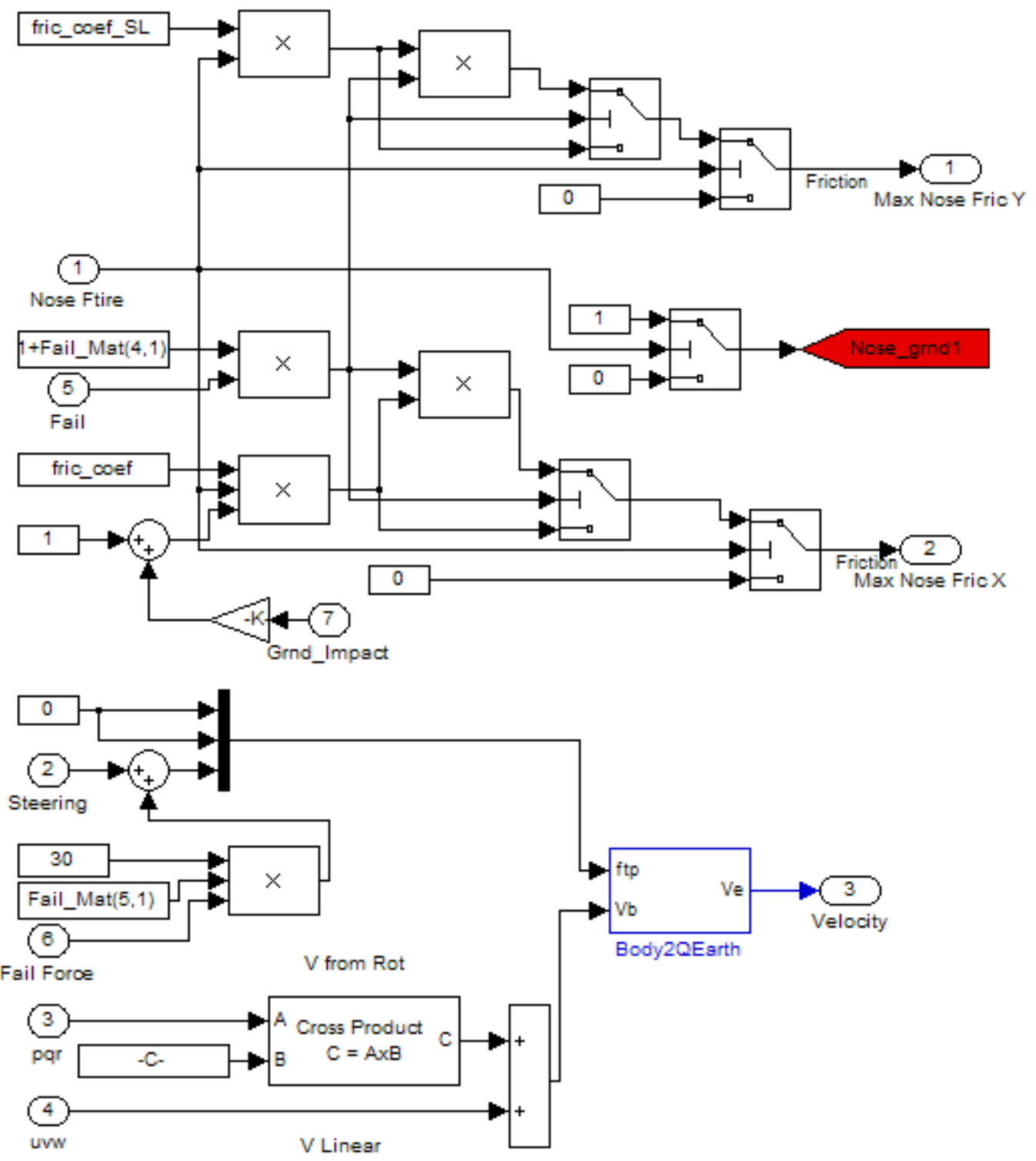

Figure 19. Maximum friction and velocity calculation. 


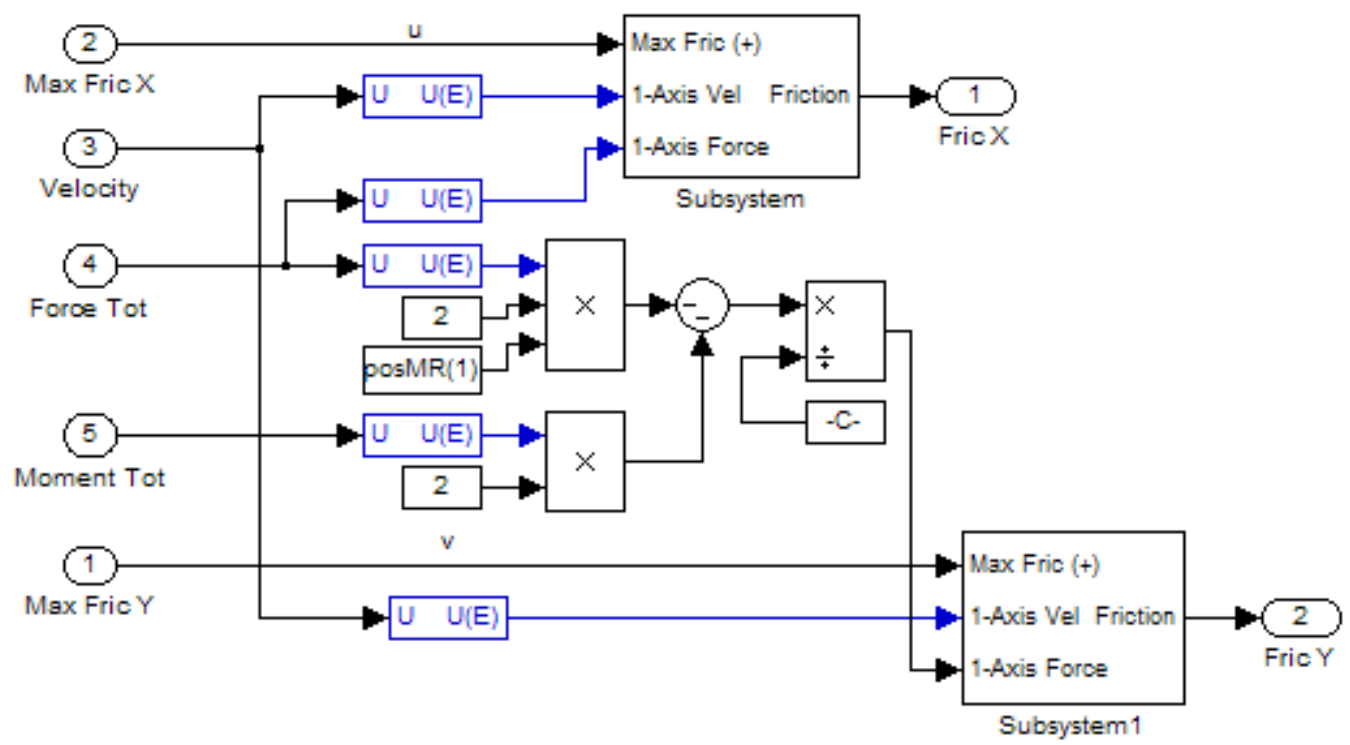

Figure 20. Routing the frictional forces for balanced motion.

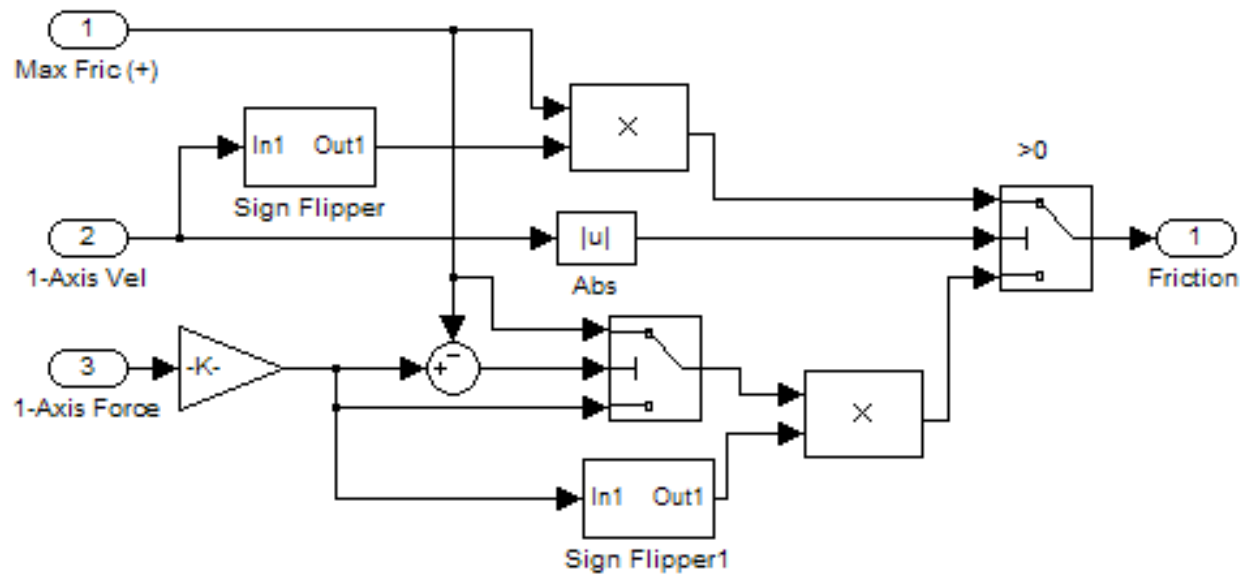

Figure 21. Applying the frictional force algorithm.

\section{Area 5 Discussion:}

This fifth element of the frictional calculation is the last step in modeling the landing gear system when subjected to normal flight conditions, combining the total forces and moments. Summing of the total forces can be seen in Figure 14. Figure 22, shows how the moments created by the landing gear are totaled. First, additional force can be applied directly to the tire from a blowout by the fourth input to the block. After 
that the moment is created by crossing the position vector for the strut with the total force created by that strut. The moment generated by each strut is divided into its three components so that a scope can be put on it to see the results more easily.

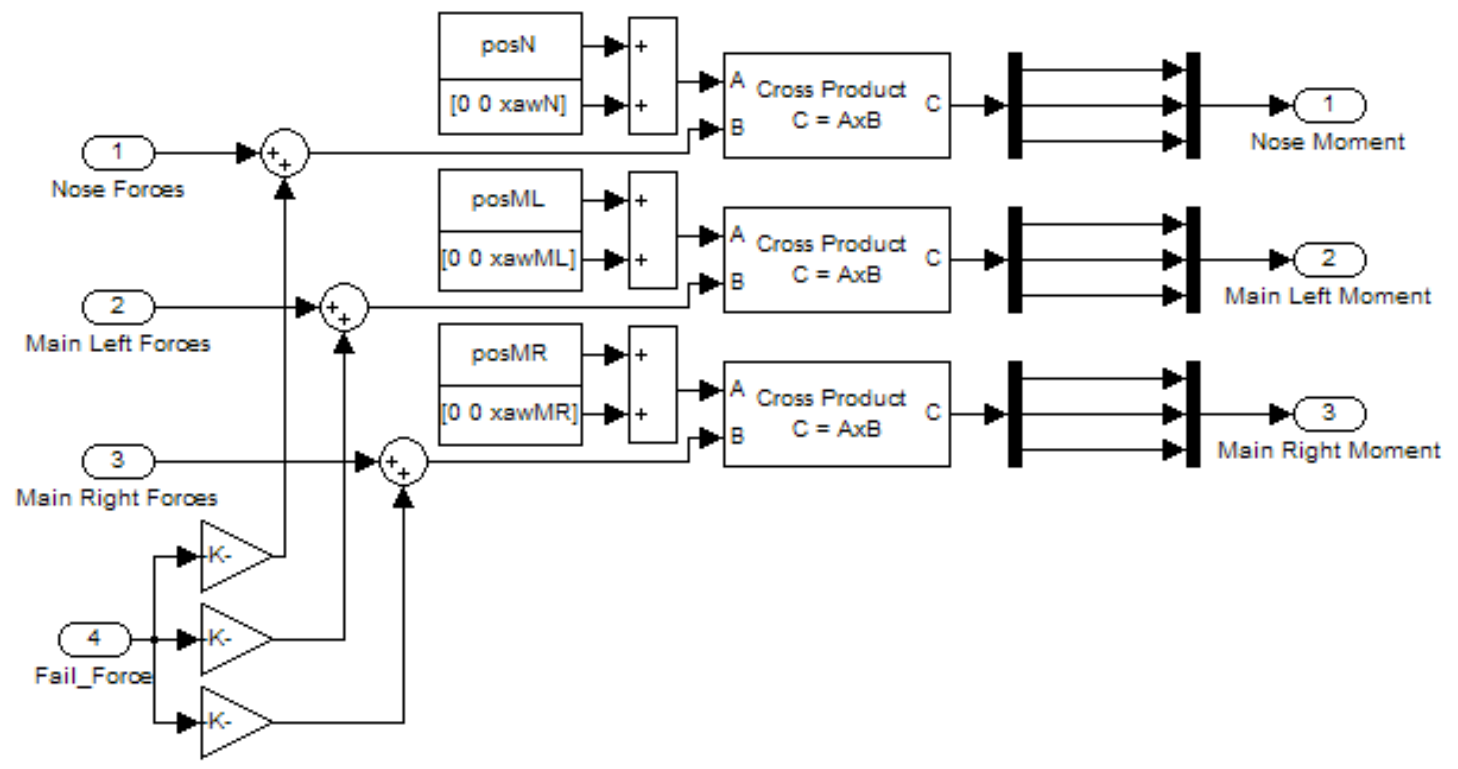

Figure 22. Summing of the landing gear's moments.

Area 6 Discussion:

In this area, the application of failure aspects is determined. Examining the area under discussion shows that this system has two triggering subsystems, the failure triggering block to the far left and the landing gear blowout force trigger. Failures can be triggered in one of three ways, to be initially failed, failed by the Real Time mode or after a certain amount of time has passed after coming into contact with the ground. This last triggering block contents is displayed in Figure 23. Input to this block is a flag that determines whether or not the airplane is in contact with the ground. The previous input to the block is the subtracted from the current input. This yields a single spike of input instead of a step input. Multiplying the clock time against this input gives the time of 
contact. If the difference between the clock time and the contact time is larger than the time for the failure after contact, the model will fail. Aft_Cont, after contact, must be a true statement in the Boolean sense. Logic similar to this is displayed in Figure 24 as to determine whether the tire is experiencing a blowout or not.

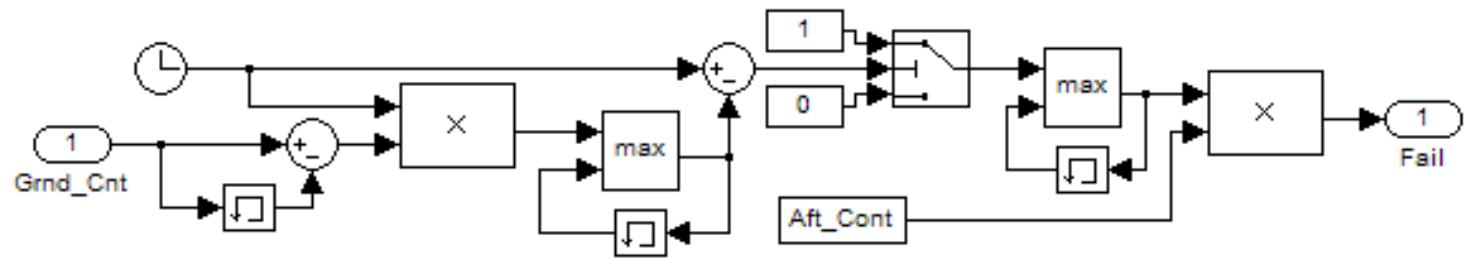

Figure 23. Technique for using timed failure trigger.

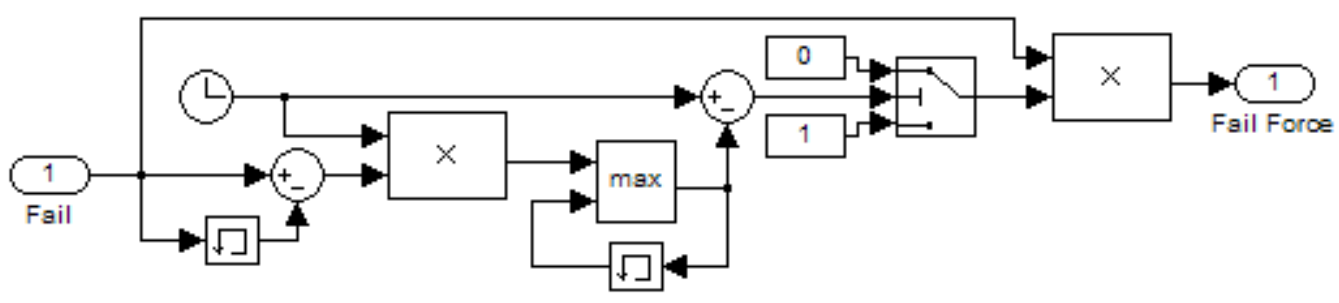

Figure 24. Technique for determining tire blowout.

\section{Area 7 Discussion:}

This last area covers failures not modeled in this thesis. The purpose of this block is to determine if the extremities of the aircraft come into contact with the landing surface. Any point on the aircraft can be tracked as long as the components of the position vector between that point and the $\mathrm{CG}$ are known in the body RF. For testing purposes, only the tips of both wings, the tip of the tail, and the bottom of the nose were considered. The logic for determining if contact occurs is identical to determining $\left|\overrightarrow{Z_{a} Z_{g}}\right| \cdot Z_{\mathrm{a}}$ is the point under consideration and contact occurs if $\left|\overrightarrow{Z_{a} Z_{g}}\right| \leq 0$. 


\section{Simulation User Interface}

The user interface is divided into two main modules: Failure Simulator and Landing System Design. The Failure Simulator module is used for students to gain flight experience for when the aircraft suffers failures upon landing. Landing System Design is a useful tool for aircraft developers to use to find the vehicle responses during landing. Both of these modes are intended to be used in an academic aspect. A series of graphical user interface (GUI) menus allow for the simulation scenario setup as described next. The first GUI encountered can be seen below in Figure 25. A couple more GUI's will be included in the body of this thesis with the rest residing in the Appendix. A flowchart of the different GUI blocks follows in Figure 26. The model will be broken down further to go into greater detail of each section.

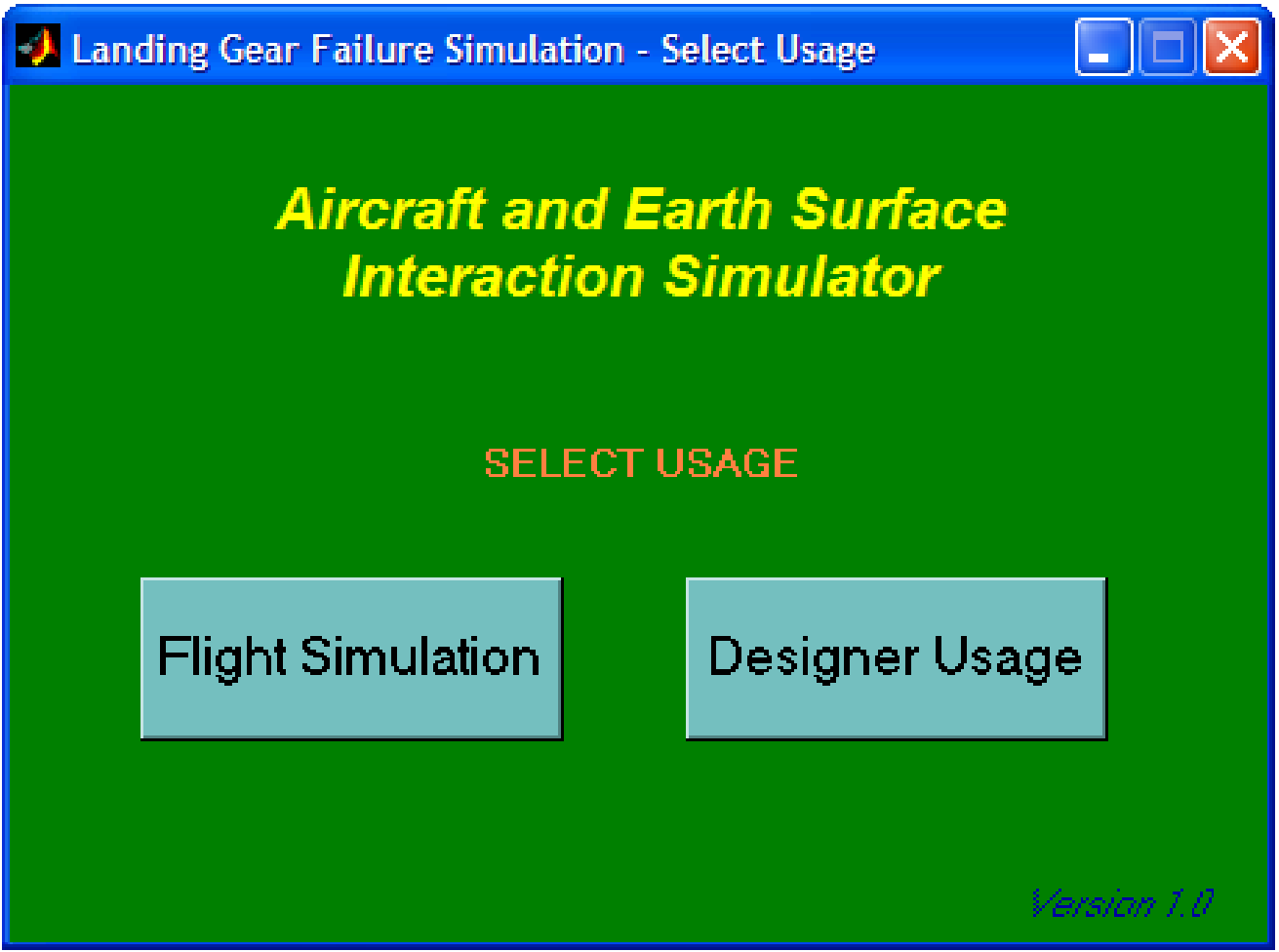

Figure 25. First GUI in employing the simulator. 


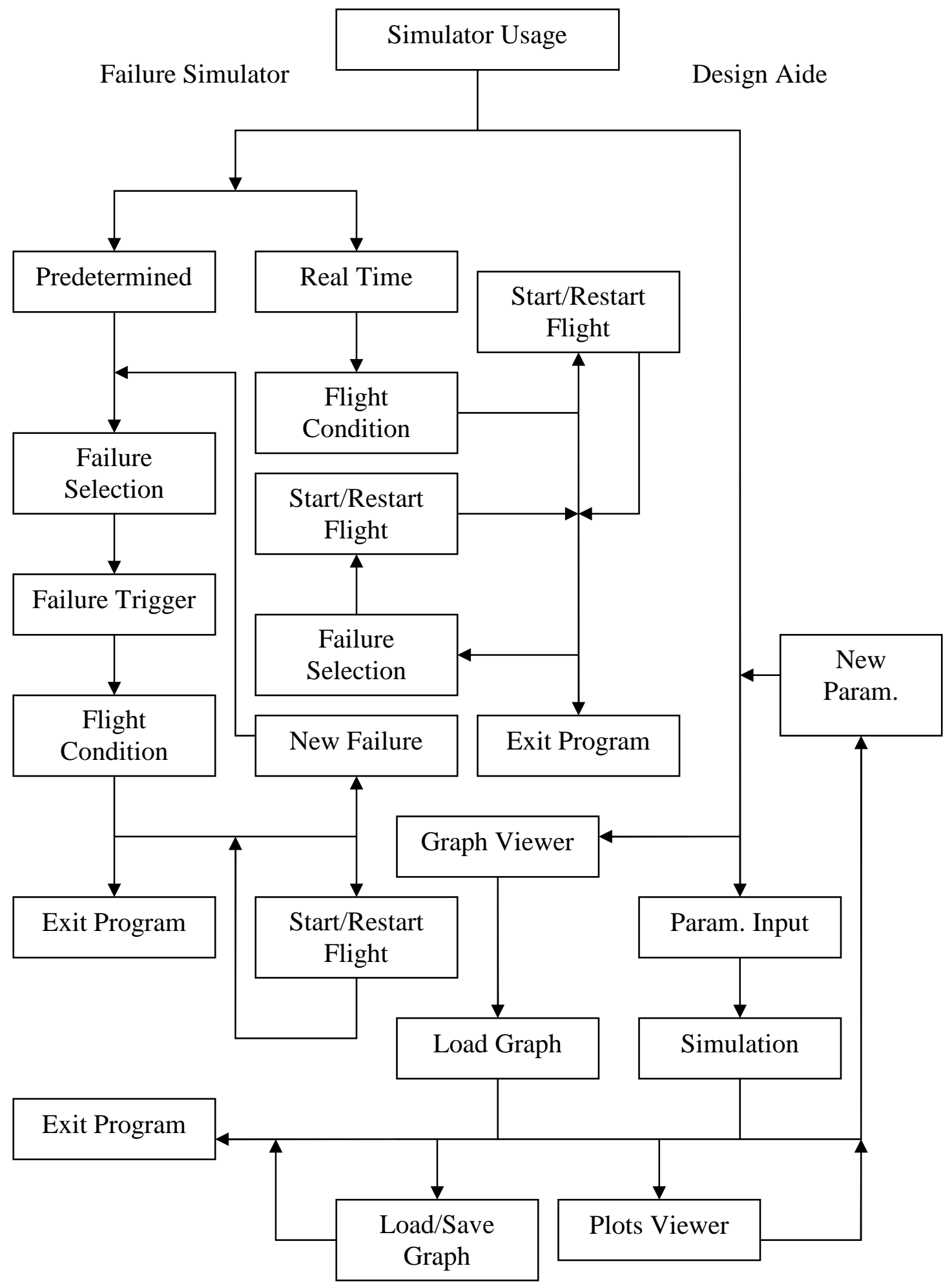

Figure 26. Flowchart of the entire simulator user interface. 


\section{A. Failure Simulator}

Once this option is chosen, there are a number of steps that need to take place prior to a flight simulation taking place. The first decision that needs to be made is how the failure will be instituted. The two options are to either have the failure take place at a predetermined time or for it to occur whenever the user decides it. The predetermined option is used to choose a failure condition and have it take place at a point in time relative to the aircraft coming into contact with the landing surface. Real time options are used so that someone observing the pilot can initiate a failure at their digression. This option is shown below in Figure 27.

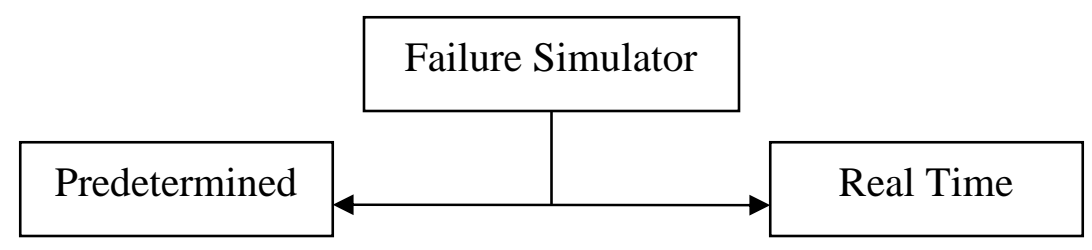

Figure 27. Opting between predetermined and real time failure initiation.

With predetermined failures selected, the next step is choosing on which strut the failure will occur. Failure can occur on either the nose, the left main, or the right main landing struts. Once the location of the failure is known, the type of failure needs to be selected. The list of all the failures applicable to each strut can be found in the prior failure section and in Figure 28 for the nose. In case the user desires to switch the location of the failure, there is an option to return to the previous screen without selecting a failure. Additionally, "no predetermined failure" can also be selected. This option is used to allow the pilot basic flying experience. This logic is portrayed in Figure 29. 


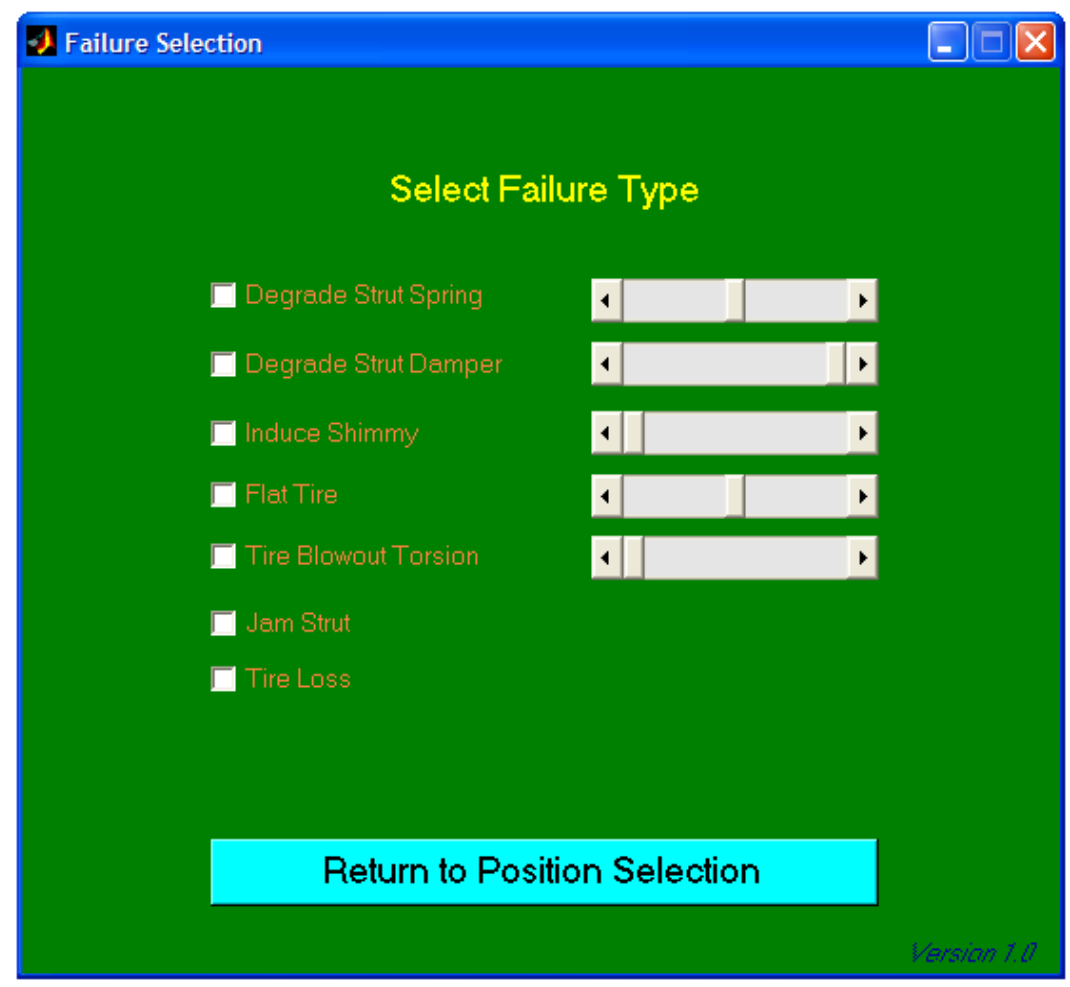

Figure 28. Depiction of possible nose failures.

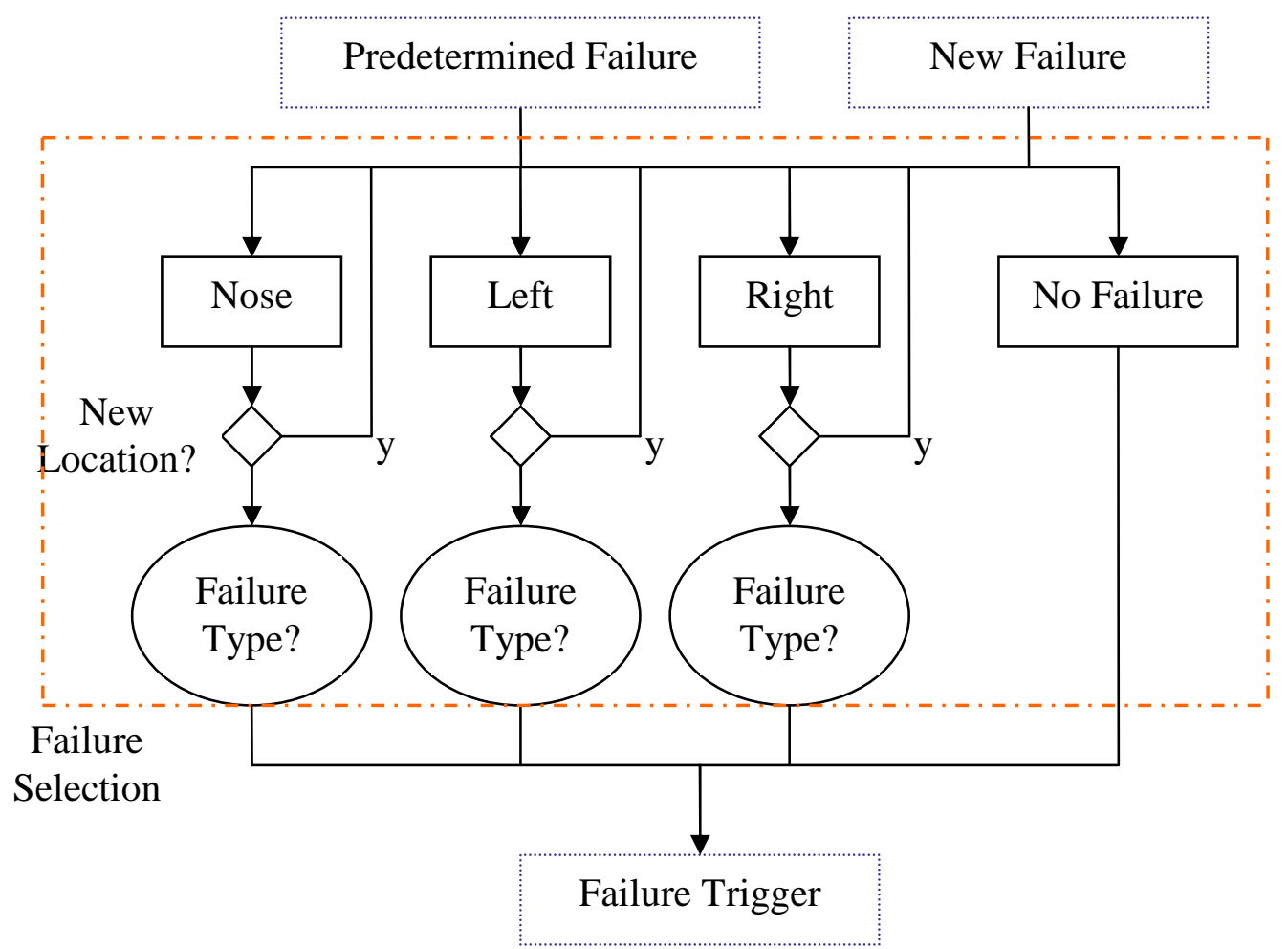

Figure 29. Selecting the location of the failure in the predetermined case. 
Once the failure type is chosen, the failure trigger needs to be selected. The two options are for the failures to occur upon initialization of the program or after a certain amount of time has elapsed once the aircraft has come into contact with the landing surface. Injecting the failure into the system upon initialization models the event of the failure being generated by the deployment of the landing gear if the model starts with the aircraft flying through the air. If the model starts on the landing surface, initialized failures model the event of the pilot missing a malfunction during the preflight check. Tying the failure into the aircraft coming into contact with the ground allows for this to be the cause of the scenario or vibrations of the landing gear being used causing it. Additionally, at this point, pilot generated failures can be elected to be used even if the user opted not to choose a predetermined failure. This works by allowing the user to choose three threshold values. The first value correlates to a maximum amount of force that the tire can be subjected to prior to bursting. The second threshold value is the amount of force needed to jam the strut and keep it from moving. Above this threshold is an additional oleo threshold; this one corresponding to the amount of force needed to sever the strut from the aircraft. These thresholds are determined by the parts of that apparatus. Examples include the load an actuator can withstand or maximum tire pressure. It has been shown that a business jet's main landing gear can fail when subjected to $39.5 \mathrm{kN}$ of force ${ }^{[31]}$. Using the option will teach pilots to land gently. Figure 30 shows the logic segment corresponding to failure condition selection. 


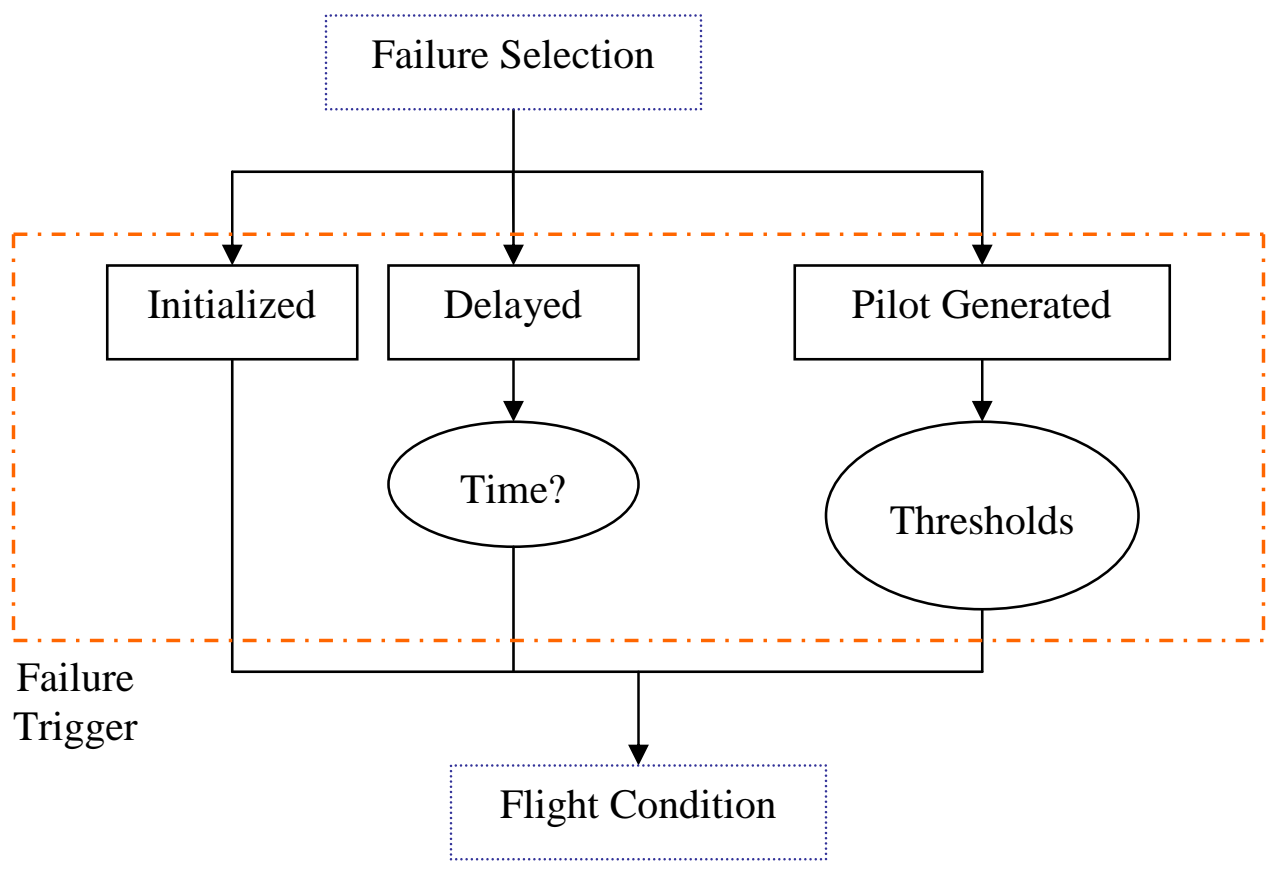

Figure 30. Selecting the failure trigger.

With the selections for failure triggers and pilot generated failures have been made, the time for flying finally comes. Before the flight can start, one more selection needs to be made; the initial flight condition for the aircraft must be selected. The three options for where the plane can start are as follows: on the runway, short approach, and far approach. Starting on the runway allows the pilot to experience taking off with the aircraft. Short and long approach options start with the plane about to land. Long approach is the same as short, but further away along the landing flight path. Once the initialization point is chosen, flight controls are open. The flight controls allow the flight to be started/restarted, a new predetermined failure selected, or for the program to be exited. The last of the predetermined logic structure is shown in Figure 31. 


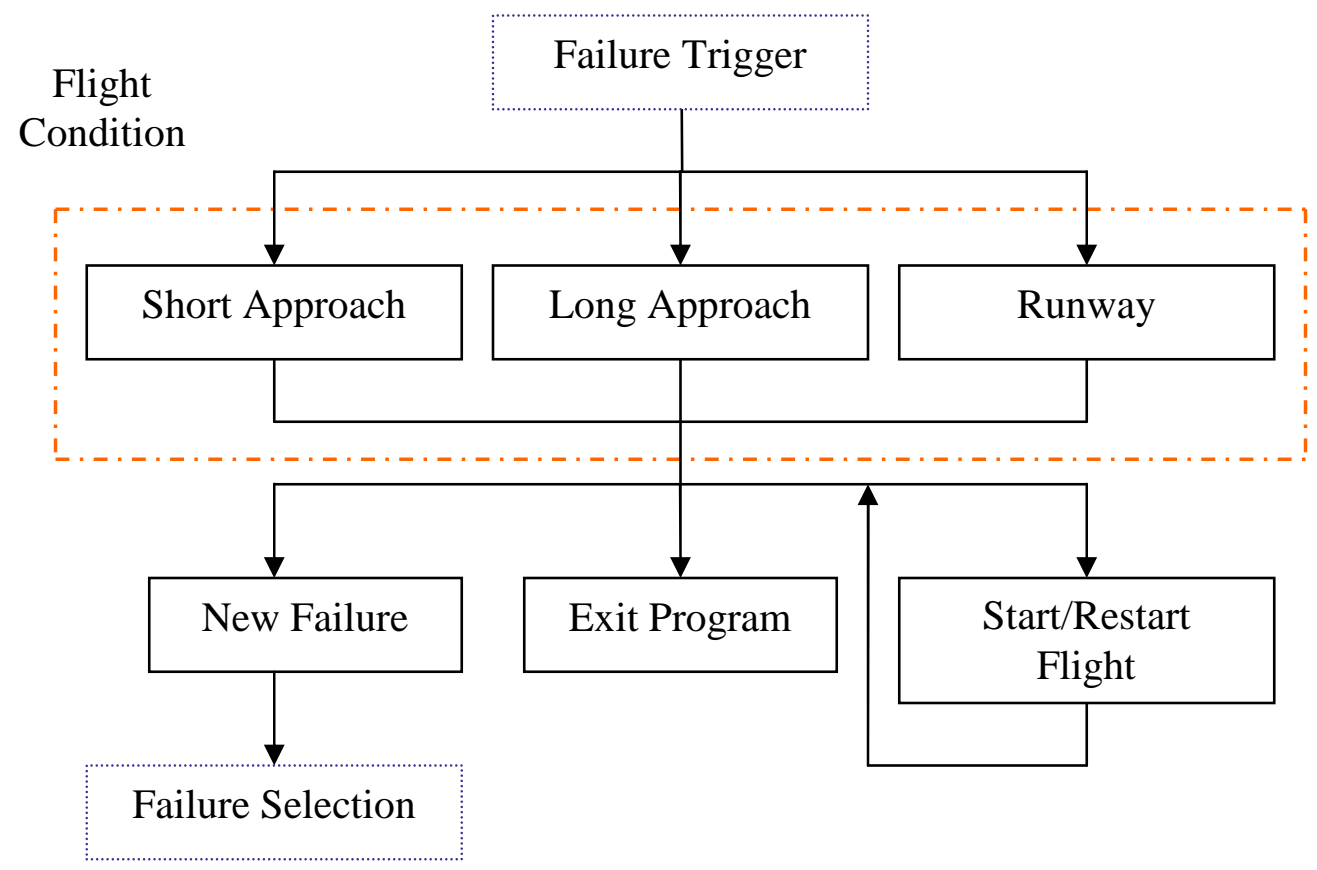

Figure 31. Starting the simulation

The real time flight simulator operation is very similar to that of the predetermined operation with a few minor differences. First, the flight condition is selected right after the real time scenario is selected. Next the flight controller and the failure selection system is combined. This is expected since there is no triggering event for the real time operation. This yields the real time logic layout as is given in Figure 32 . 


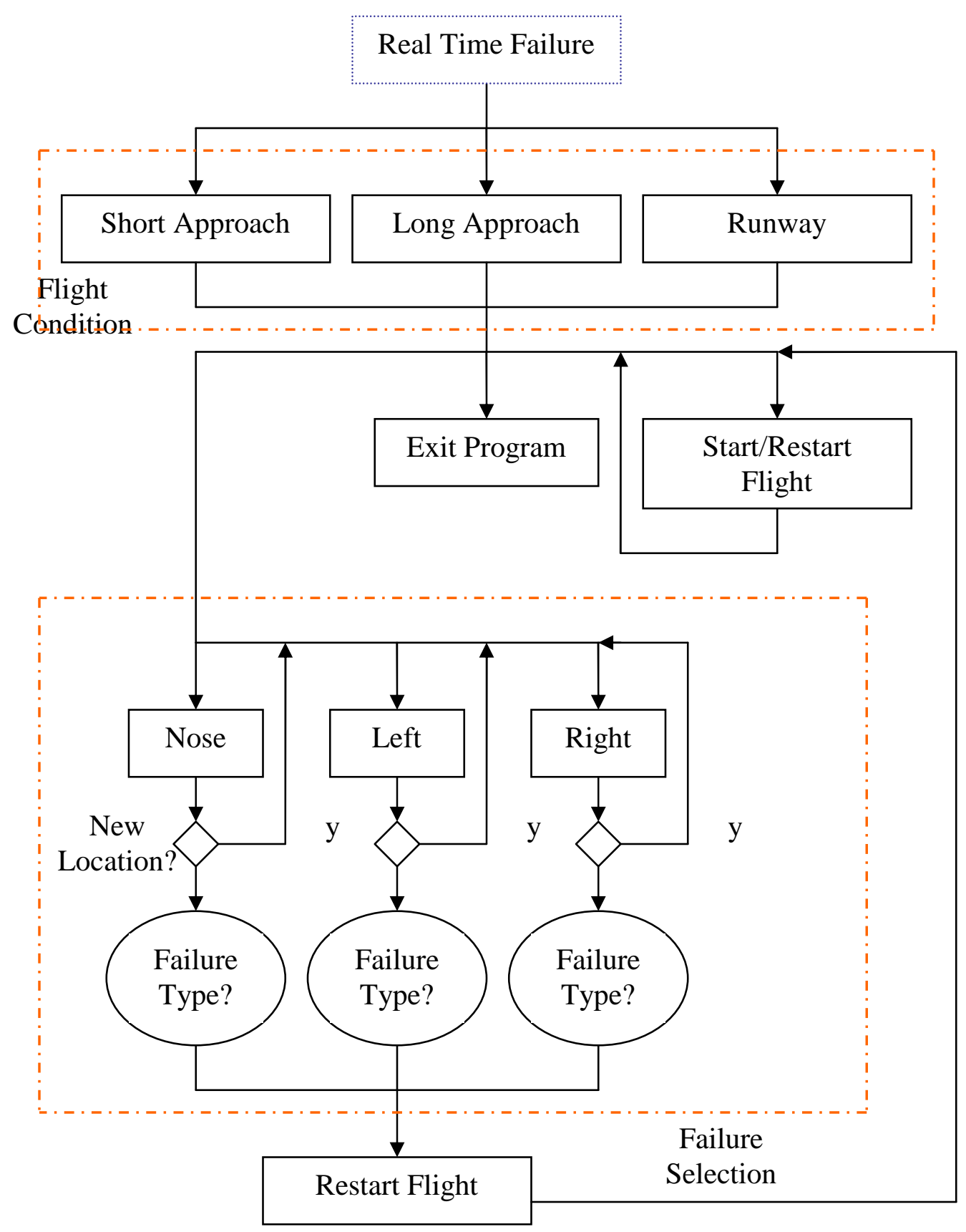

Figure 32. Control logic of the real time portion of the failure simulator. 


\section{B. Landing System Design Aide}

Once the Landing System Design option is selected, using this computational tool is completed in only two steps. These two steps are to define the test parameters and to review the results these parameters generate. A good reference for generating a test parameter set can be found from Aircraft Landing Gear Design ${ }^{[32]}$.

In the first phase of the landing design tool, a lot of factors need to be defined. First, the dynamic coefficients of the aircraft, spring and damper values must be identified. Second, the unloaded position of the wheel relative to the CG needs to be given. When this information is combined with the next entry, strut deflection angles, the SDL can be calculated. Mass of the aircraft is needed to find the dynamic response of the entire aircraft. Finishing the list of variables that affect the dynamics of the landing gear, the radius of each tire and the effective mass of the landing gear must be given. The last aircraft parameter is the frictional force the aircraft generates while landing. These values can be derived from the geometric layout and component data of the aircraft.

The initial flight condition parameters round out the last of the necessary inputs to the system. Height above the landing surface for the aircraft to initialize with is the first needed input. Initial Euler angles are the next entries. Downward and forward velocities round out the list of variables that must be defined to create the initial flight condition. These parameters are necessary to serve as a basis that the results can be compared against. To generate these results, the desired run time for the model needs to be given. With the current computational abilities of computers, each second of run time can take dozens of seconds to be calculated. The GUI where these parameters are entered is shown in Figure 33. 


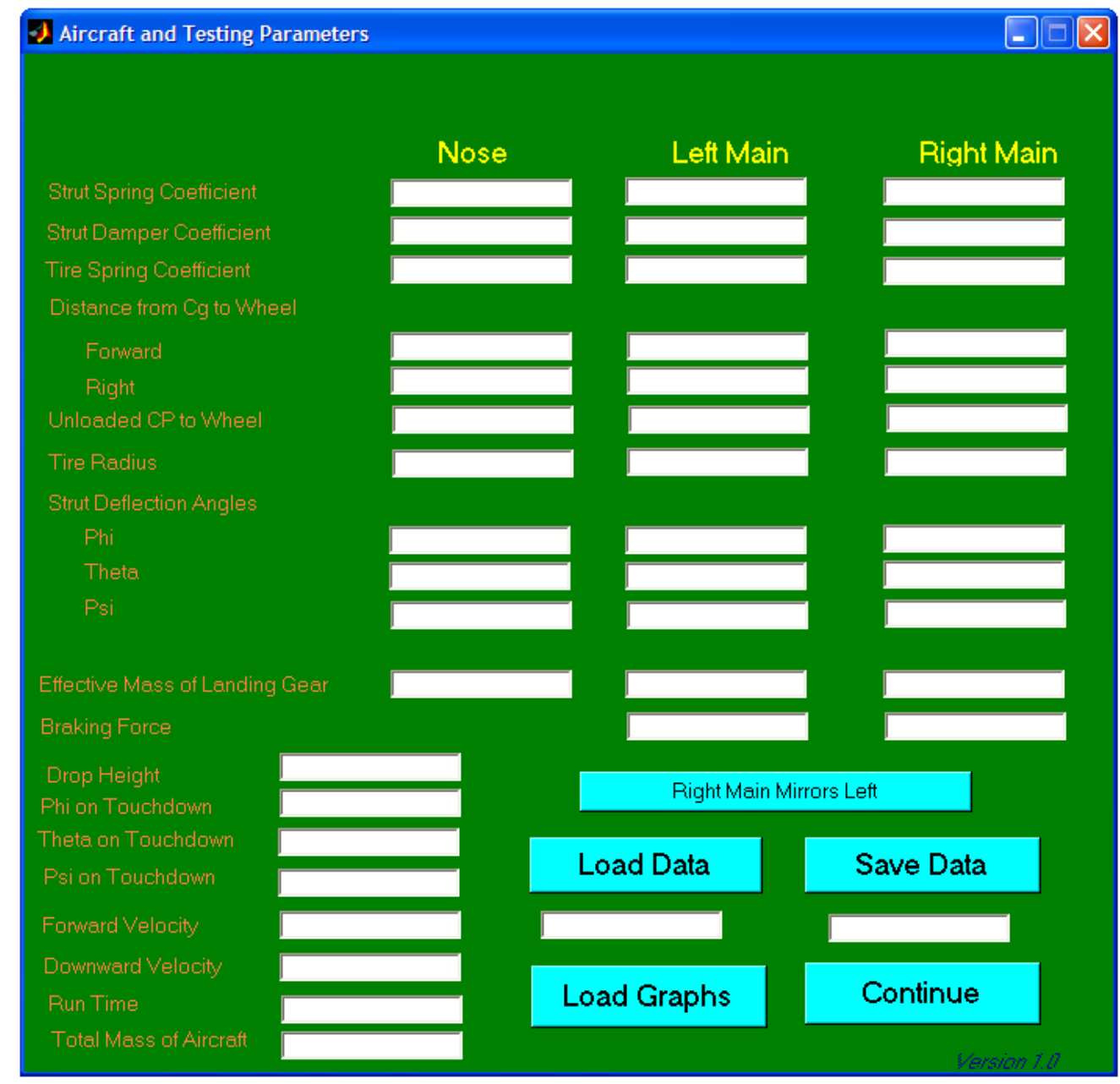

Figure 33. GUI used to input design aide parameters.

To make using the designer mode of the simulator easier, several helping features are added. Since most aircrafts are symmetric entities, the option to make the right main landing gear mirror the left is given. This cuts the number of entries by twenty-five percent. If all of the entries want to be skipped, the ability to load and save data can be utilized. If the results from a past running of the designer computational tool are to be viewed, the graph viewing portion of the program can be jumped to. Using this option needs the results from a previous test to be loaded. Running the computational tool simulates the aircraft's flight assuming no pilot input. The flowchart for this is shown in Figure 34. 


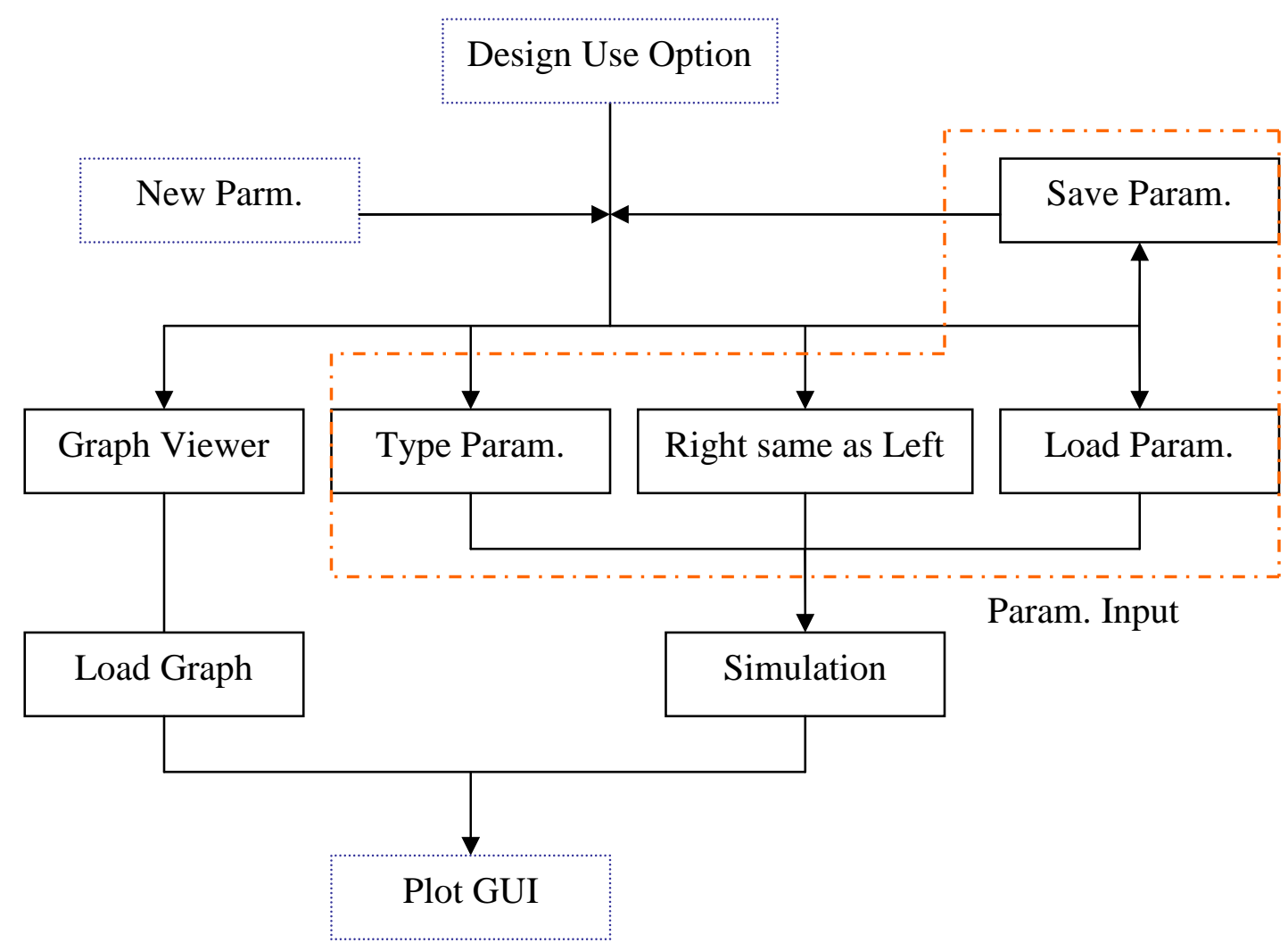

Figure 34. Data entry techniques for designer tool.

At this point, the dynamic response of the landing gear system can be analyzed.

These responses can be seen in the plots of some of the aircraft states, $\left|\overrightarrow{Z_{a} Z_{w}}\right|$ and $\left|\overrightarrow{Z_{a} Z_{g}}\right|$.

The Euler angles and velocity components show how the entire aircraft responds to the given landing gear parameters. How each landing gear responds to the landing gear parameters can be viewed by examining the $\left|\overrightarrow{Z_{a} Z_{w}}\right|$ plots and its single and double derivatives for each strut. $\left|\overrightarrow{Z_{a} Z_{g}}\right|$ and its associated plots can also lend information on the dynamic analysis of the landing gear systems. Figure 35 shows the options of the GUI depicted as a flowchart. 


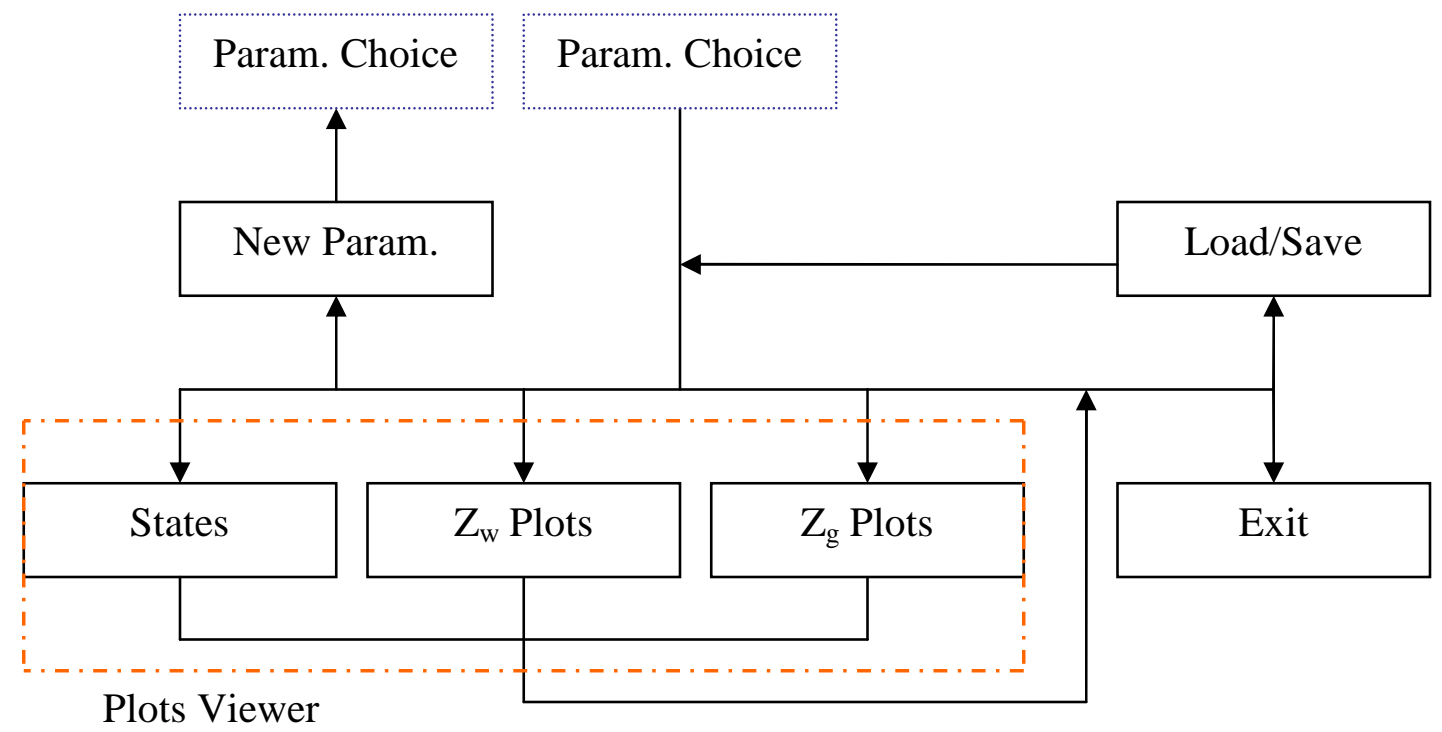

Figure 35. Graph plotting logic for designer tool. 


\section{Results}

\section{A. Normal Conditions}

A1. Stationary Tests:

The tests of this section are those that were gathered while the model simulated the aircraft when no thrust was applied. The model starts with the aircraft being released from a stationary position when the three wheels of the landing surface are coming into contact with the landing surface. From the next five figures, (Figure 36-40) the normal response of the landing gear system is displayed. These graphs have the following steady state values. The altitude of the aircraft is just below $315.15 \mathrm{~m}$. Pitch angle (theta) is $1.9^{\circ}$. The magnitude of the force generated by the nose gear is slightly less than $4,000 \mathrm{~N}$. The main gears generate a force with magnitude of $28,500 \mathrm{~N}$. The reason that the results are shown with a negative value is due to the positive $\mathrm{Z}$ axis direction being downward. The magnitude of the distance between the top of the landing gear strut and the hub of the wheel is $1.67 \mathrm{~m}$.

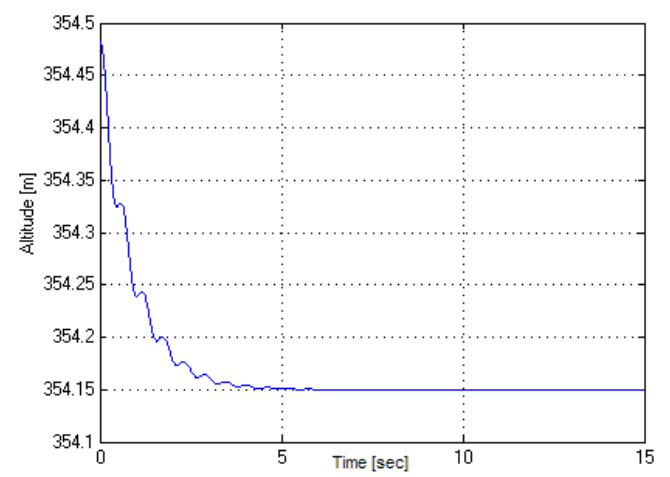

Figure 36. Altitude when subjected to normal conditions.

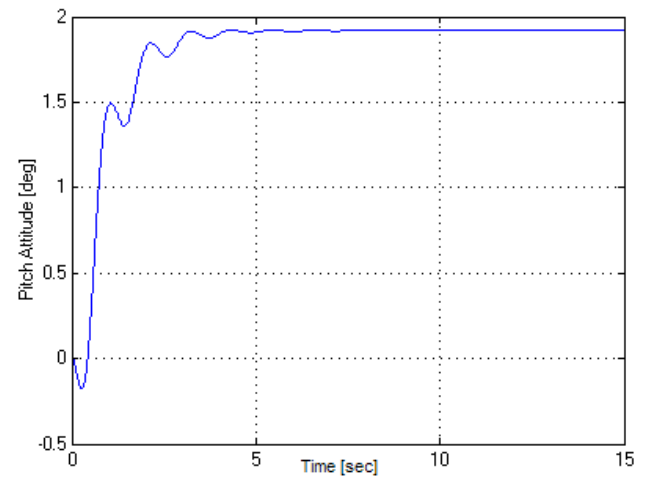

Figure 37. Pitch attitude when subjected to normal conditions. 


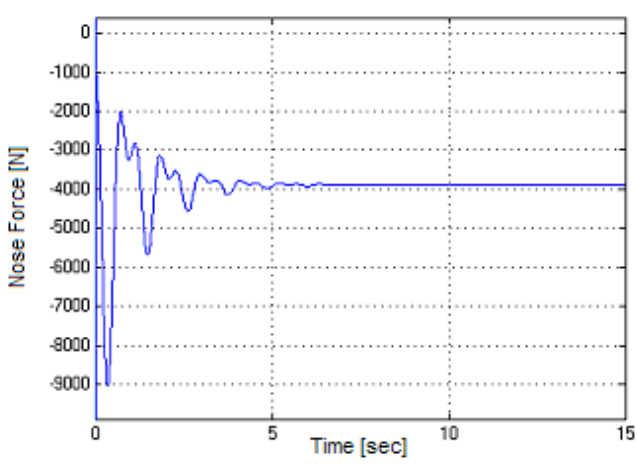

Figure 38. Force generated by the nose when subjected to normal conditions.

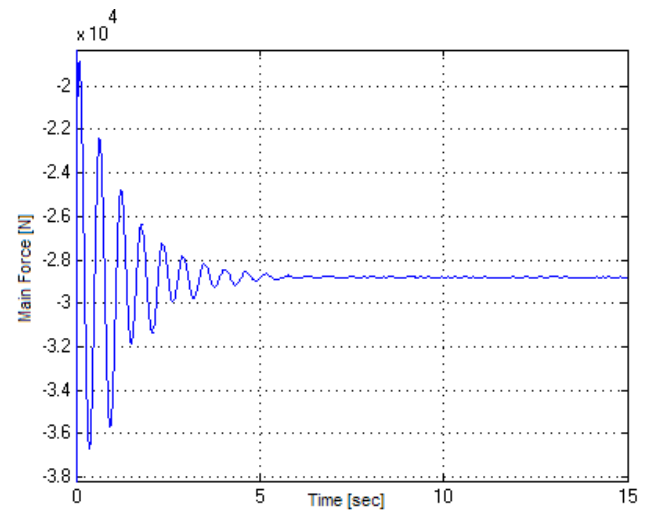

Figure 39. Force generated by the main when subjected to normal conditions.

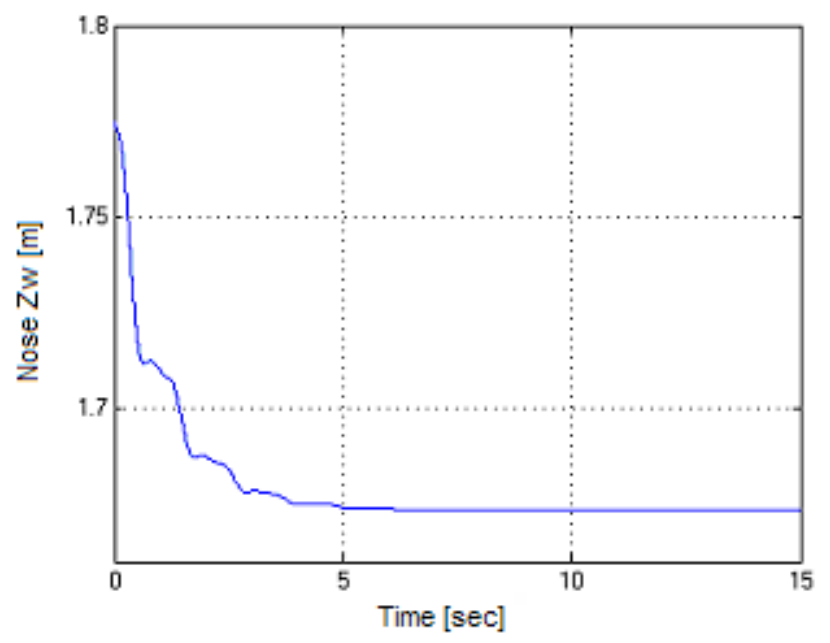

Figure 40. Distance along SDL to the hub when subjected to normal conditions

\section{A2. Moving Tests (Non-Braking):}

This section deals with tests that were generated under the same conditions as were stated as above but with half the maximum thrust of the aircraft. This source of thrust causes a few effects on the normal response of the aircraft. First of all, an acceleration of $1.1 \mathrm{~m} / \mathrm{s}^{2}$ is created as can be derived from Figure 41 . Secondly, the altitude of the aircraft is increased by only a few millimeters as seen in Figure 42. This is from the forward motion of the aircraft causing a small degree of lift on the aircraft. Figures 43-45 show the effect of having the source of the thrust above the CG. The pitch 
attitude drops to $1.4^{\circ}$ compared to the stationary $1.9^{\circ}$. This is achieved by the nose strut being compressed an additional $3 \mathrm{~cm}$. To negate the moment generated by the engines, the magnitude of the force generated by the nose is increased to over $5000 \mathrm{~N}$.

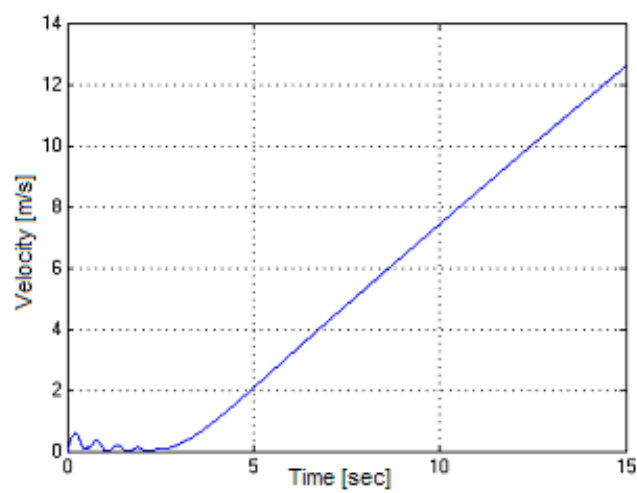

Figure 41. Velocity under normal conditions with half thrust.

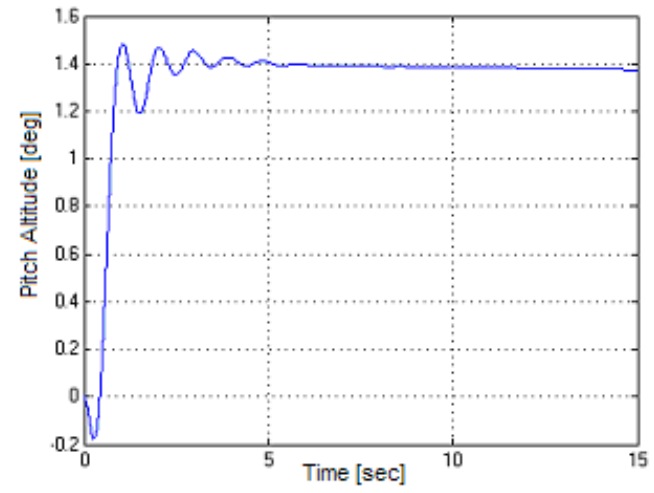

Figure 43. Pitch attitude under normal conditions with half thrust.

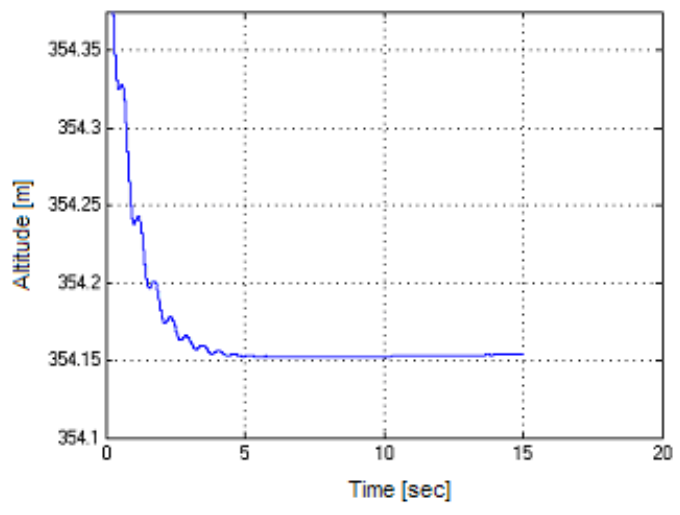

Figure 42. Altitude under normal conditions with half thrust.

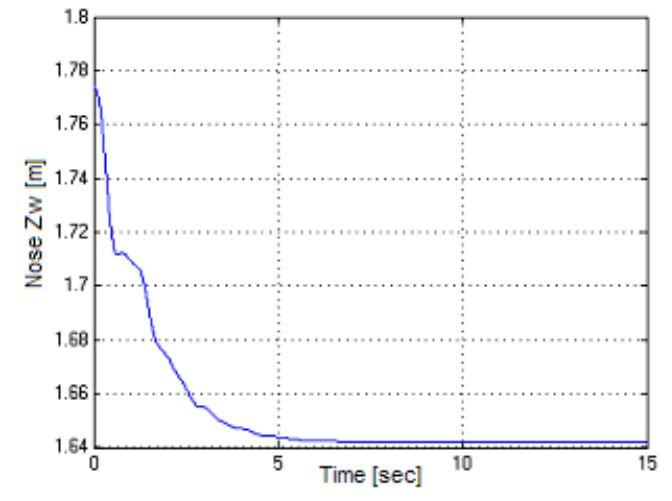

Figure 44. Distance to the wheel hub under normal conditions with half thrust. 


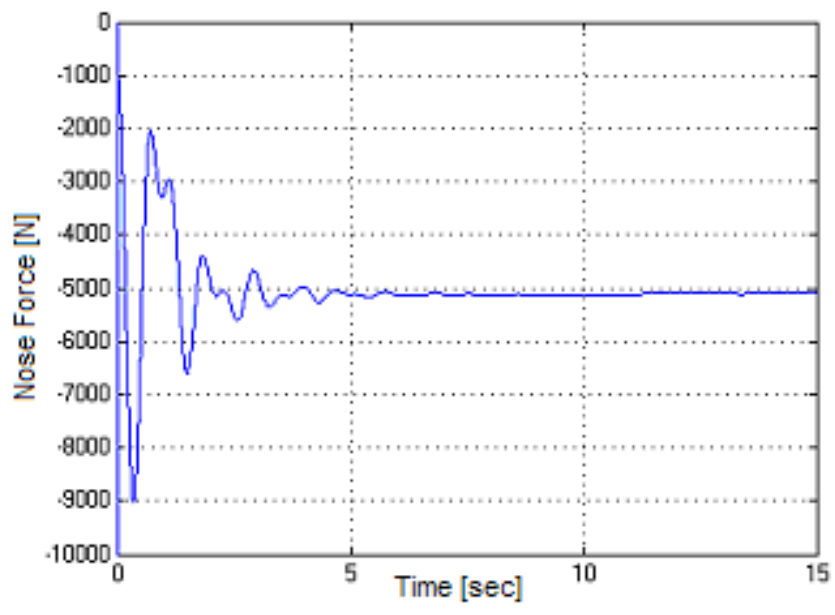

Figure 45. Force generated by the nose under normal conditions with half thrust.

\section{A3. Moving Tests (With Brakes):}

The last subsection deals with the simulation of failures to the braking system. To test the following scenario, a constant $25 \%$ braking capability was applied to the aircraft from the initial conditions as stated in the previous section with one exception. The thrust applied to the aircraft was raised so that the acceleration of the aircraft mirrored that as was the case without the brakes being applied. Only a small portion of the braking capability was applied since the aircraft is designed to not take off when the brakes are fully applied. Under normal conditions, it is found that one of the aircraft main struts can produce at steady state $4,750 \mathrm{~N}$ as seen in Figure 46 with this lower braking capability. The oscillation of available braking force is an effect of the aircraft being initiated when it is just coming into contact with the landing surface. The load on the main struts has yet to reach steady state. Accelerating while the brakes are applied is done to show the effect that failure of the braking system has on the model. 


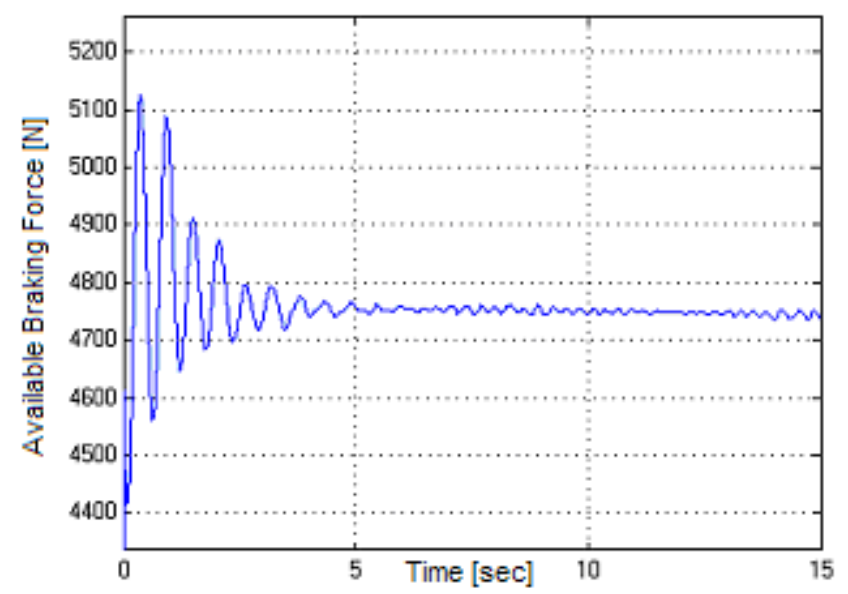

Figure 46. Available braking force with only $25 \%$ brakes applied.

\section{B. Abnormal Conditions}

\section{B1. Stationary Tests:}

Strut Spring Degradation:

The following set of results is generated by lowering by half the nose oleo spring coefficient. Degrading failures would most often be a general change caused by eroding effects but can also occur instantaneously. For demonstration of this failure, the abnormal condition was instituted at the two second mark. It can be seen in Figure 47 that the altitude is lowered slightly by reducing the spring's effectiveness. This change in altitude would be greater if the failure would happen to occur on one of the main landing struts since they reside closer to the CG. In Figure 48 it can be seen that the pitch angle is lowered to $.75^{\circ}$. Since the spring coefficient is lowered, the strut needs to compress further, causing the aircraft to angle more downward. This effect can also be seen in Figure 49 with the distance to the wheel hub being only $1.56 \mathrm{~m}$. This implies that the strut needs an additional $11 \mathrm{~cm}$ to decompress. Figure 50 shows a side-effect of the aircraft being angled downward more. The steady state force generated by the nose strut 
is closer to $-4200 \mathrm{~N}$. The lower pitch of the aircraft causes the CG to move closer to the nose of the aircraft. The spike at the two second mark is from the damping portion of the oleo causing a larger force.

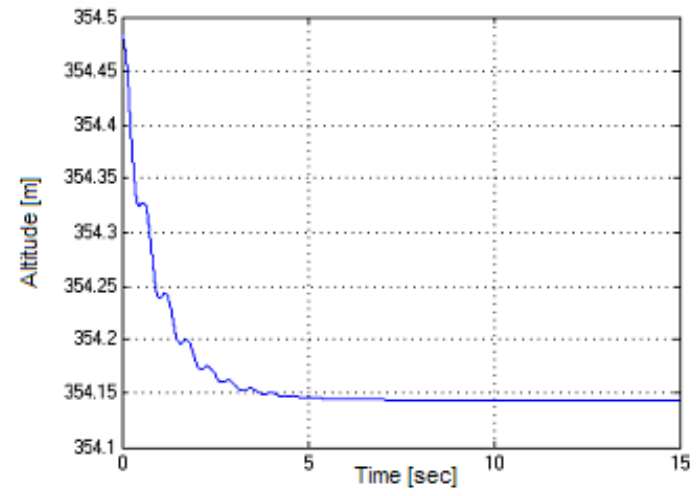

Figure 47. Altitude when subjected to a degraded strut spring.

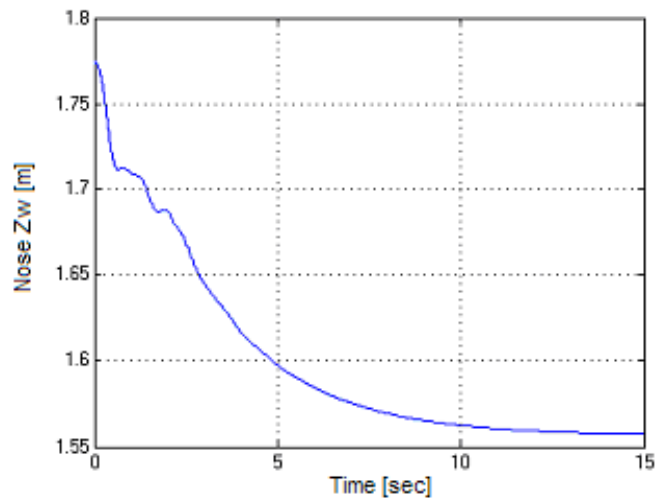

Figure 49. Distance along the SDL to the hub when subjected to a degraded strut spring.

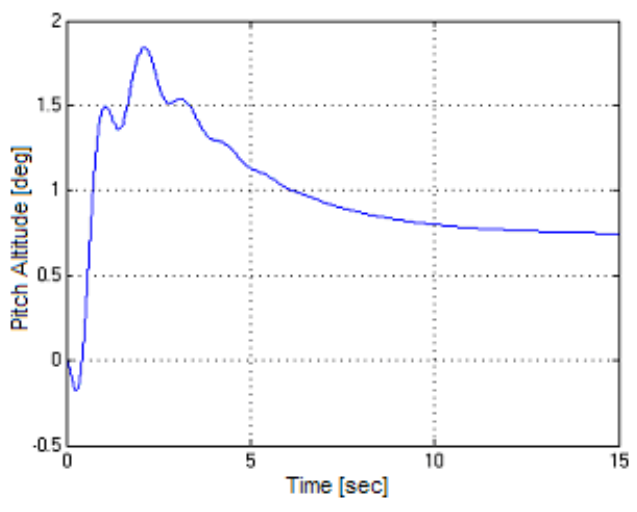

Figure 48. Pitch angle when subjected to a degraded strut spring.

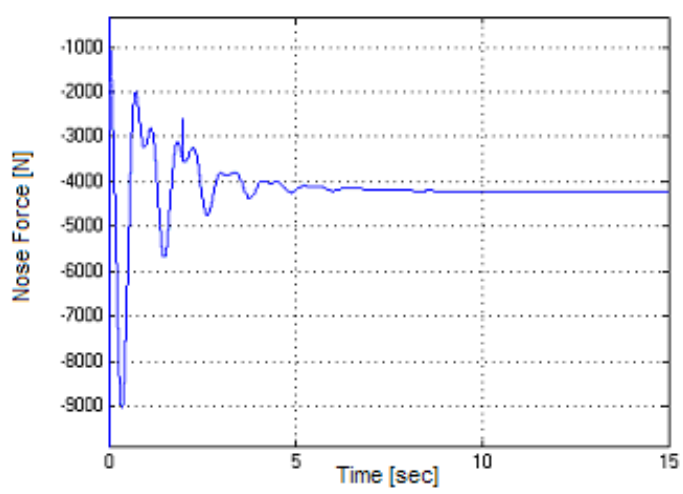

Figure 50. Force generated by the nose when subjected to a degraded strut spring.

\section{Strut Damper Degradation:}

The steady state values of the degraded strut damper are identical to that of the aircraft when it was subjected to normal conditions. Since dampers affect the velocity of systems, the same steady state is expected. The damper coefficient was cut in half at the 
2 second mark as can be seen in the spike in the data. After the failure occurs, an increase in the natural frequency can be seen by examining Figure 51, the main landing gear force graph. In the normal conditions the natural frequency is $2 \mathrm{HZ}$. With a degradation of the strut damper, the natural frequency is $1.6 \mathrm{~Hz}$. The effect of lowering the damping coefficient of the nose gear has a negligible effect in respect to the force generated. In figures 52 and 53 the effect of failing one of the main strut dampers can be seen. Pitch is relatively unchanged. This could be from the other damper compensating and obscuring the failure. On the other hand, the roll attitude spikes at the time of the failure. This makes sense with the unbalance of forces.

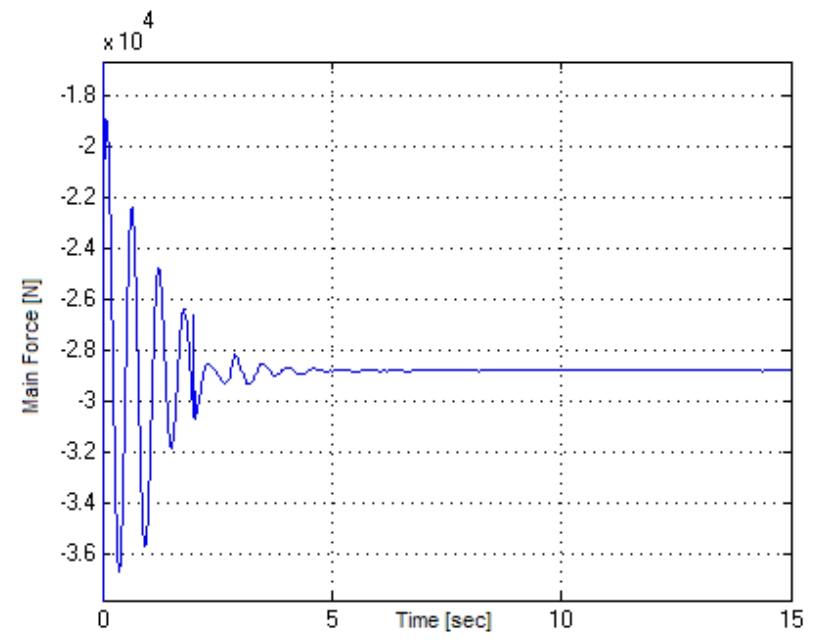

Figure 51. Force generated by the main when subjected to a degraded strut damper. 


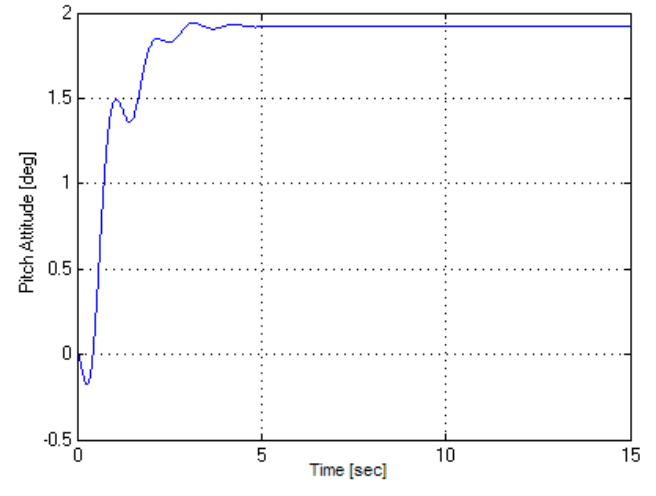

Figure 52. Pitch attitude when subjected to a degraded strut damper.

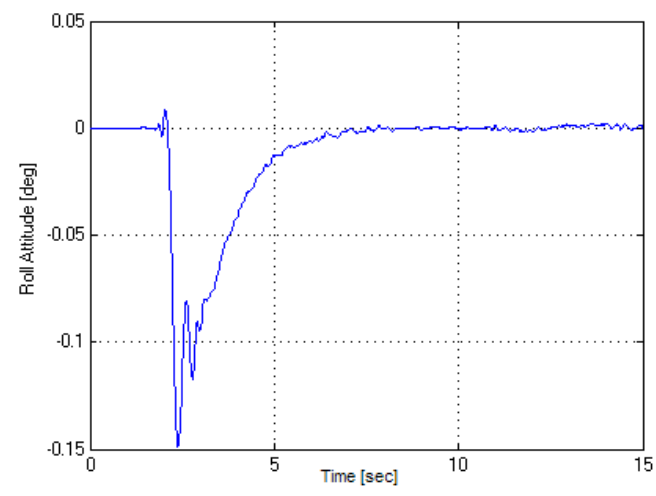

Figure 53. Roll attitude when subjected to a degraded strut damper.

Strut Jam:

Figures 54-57 contain the simulation results of jamming the strut of the nose. In all of the results, it can be noticed that the response oscillates more than the normal scenario. The increased oscillations are from the removal of a damping apparatus. During the 15 second runtime, neither the force generated nor the pitch attitude achieves a steady state, Figures 54 and 55 respectively. It can also be seen from the graph of the pitch that the nose of the aircraft is raised slightly higher into the air. Examining the wheel hub distance, the reason for this can be seen. This distance is increased by a couple of centimeters that was generated by when the strut was jammed at the two second mark. As can also be seen in Figure 56, the distance to the wheel hub is constant as it should be with a jammed strut. The increased distance is mirrored in the altitude according to the discussion earlier inversely applied. 


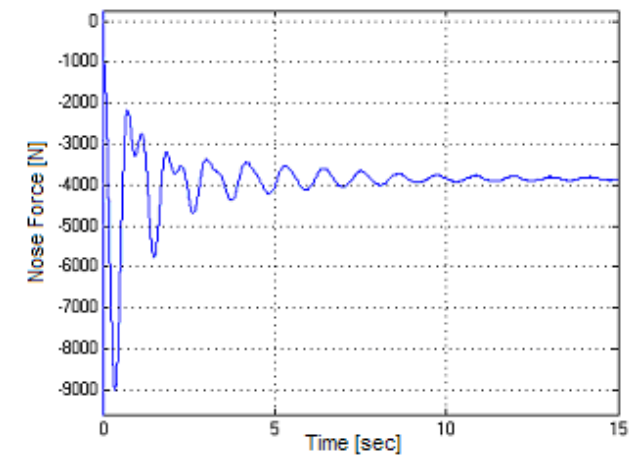

Figure 54. Force generated by the nose when subjected to a jammed strut.

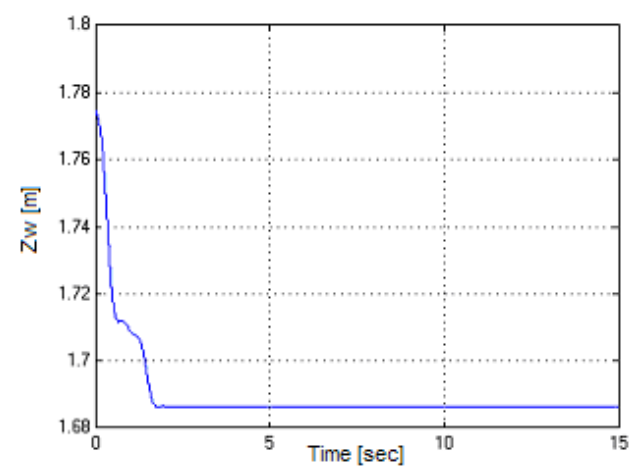

Figure 56. Distance along the SDL to the hub when subjected to a jammed strut.

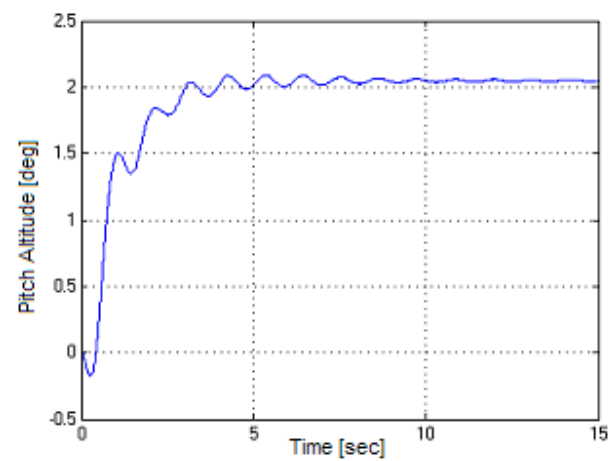

Figure 55. Pitch attitude when subjected to a jammed strut.

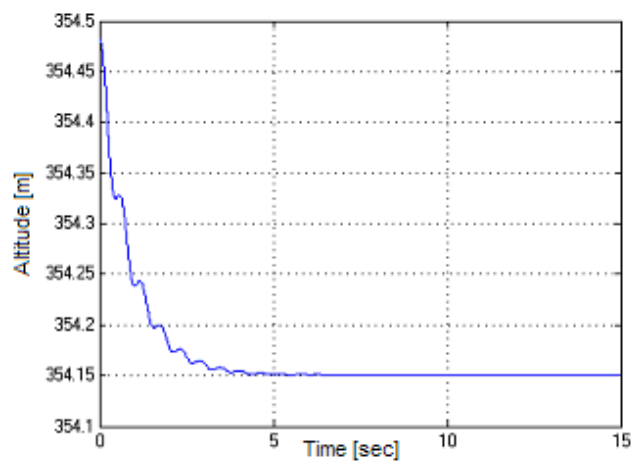

Figure 57. Altitude when subjected to a jammed strut.

\section{B2. Moving Tests (Non-Braking):}

Tire Loss:

Removing the tire from the simulation environment at the two second mark generates the following aircraft dynamic responses. First, the acceleration of the aircraft drops to $.6 \mathrm{~m} / \mathrm{s}^{2}$ as can be derived from Figure $58 \mathrm{ww}$. This result arises from the friction between the aircraft and the landing surface being generated by the sliding of steel along asphalt instead of the tire simply rolling along the surface. The pitch attitude of the aircraft is lowered to $-.4^{\circ}$ as seen in Figure 59. The removal of the tire reduces the length 
of the strut by several centimeters alone. With the shorter strut, the CG shifts forward further, causing the nose strut to be loaded to a higher degree as seen in Figure 60. This force is now raised to $6500 \mathrm{~N}$. The ending oscillation of the force could be generated by the aircraft's nose wheel touching off of the landing surface. A drop in the nose force occurs after the tire is lost since the strut is no longer in contact with the landing surface. The larger load is created by having the strut be compressed an additional $7 \mathrm{~cm}$ from normal as seen in Figure 61.

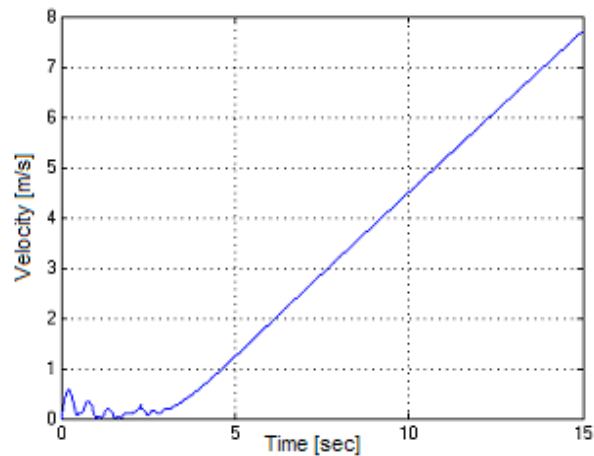

Figure 58. Velocity under half thrust with tire loss.

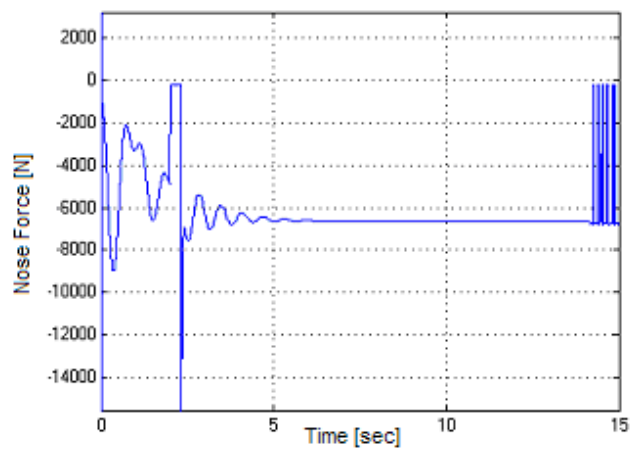

Figure 60. Nose force under half thrust with tire loss.

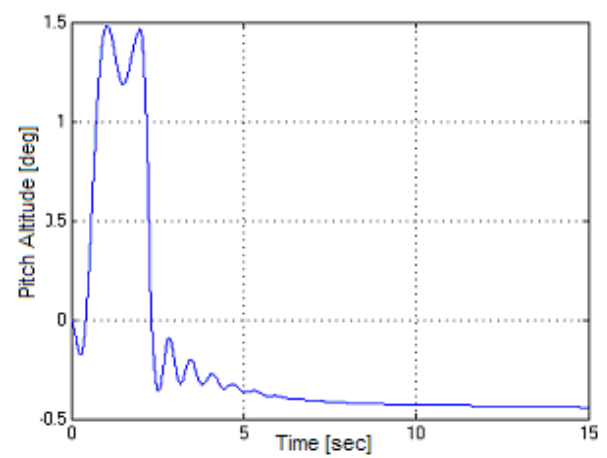

Figure 59. Pitch attitude under half thrust with tire loss.

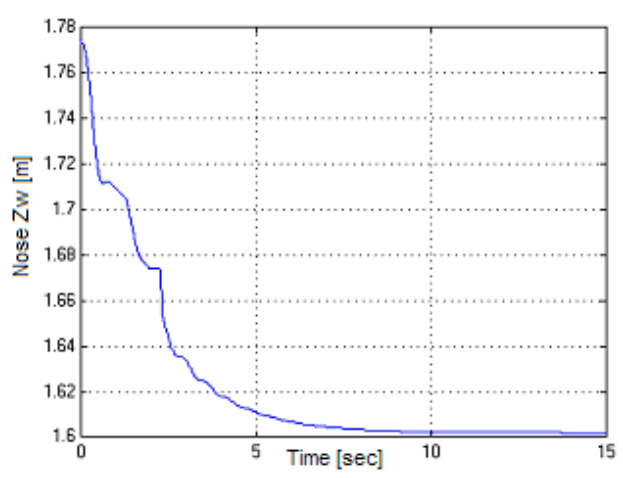

Figure 61. Distance along SDL to wheel hub under half thrust with tire loss. 


\section{Blowout:}

The dynamic response as generated by the loss of a tire is identical to that of a blowout with a few exceptions. Since this scenario was simulated to occur on the nose strut, the wheel will pivot from the exploding tire. Figure 62 demonstrates this as the turning angle reaches over half a radian. As can be seen, the event occurs at the two second mark. Also at the two second mark, a spike in the side force on the nose wheel can be seen in Figure 63. In this figure it can also be seen that the side force is applied in an oscillating manner. This is caused by the continuous landing gear system being modeled as a discrete system. By decreasing the time step, this phenomenon can be minimized.

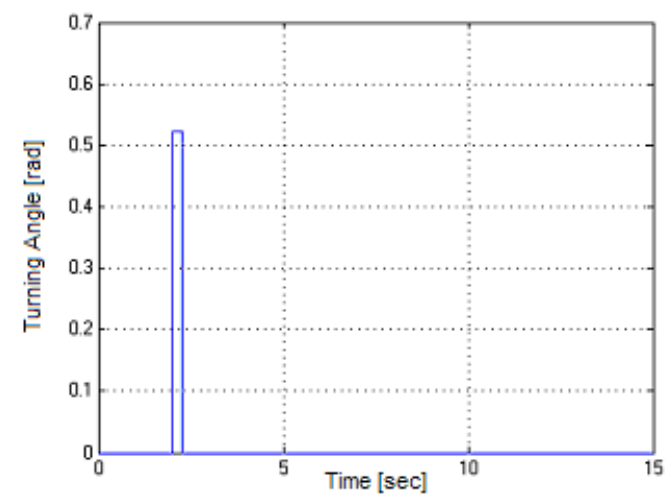

Figure 62. Turning angle under half thrust with tire blowout.

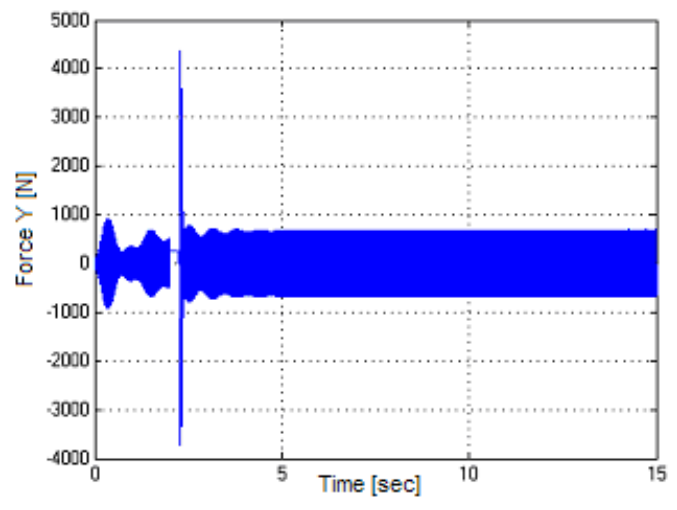

Figure 63. Side force under half thrust with tire blowout.

\section{Shimmy:}

Shimmy of the nose wheel produces two effects. The most notable is that the turning angle oscillates with a given frequency and amplitude as pictured in Figure 64. For this demonstration, the amplitude was set to be .1 rad with a frequency of $10 \mathrm{~Hz}$. This is an extreme case to demonstrate the user's ability to set the action. This effect in turn caused the aircraft to experience a rolling motion as pictured in Figure 65. As can be 
seen, the amplitude of the roll increases when the shimmy is induced at the two second failure time. Not displayed is a very slight decrease in the aircraft's acceleration.

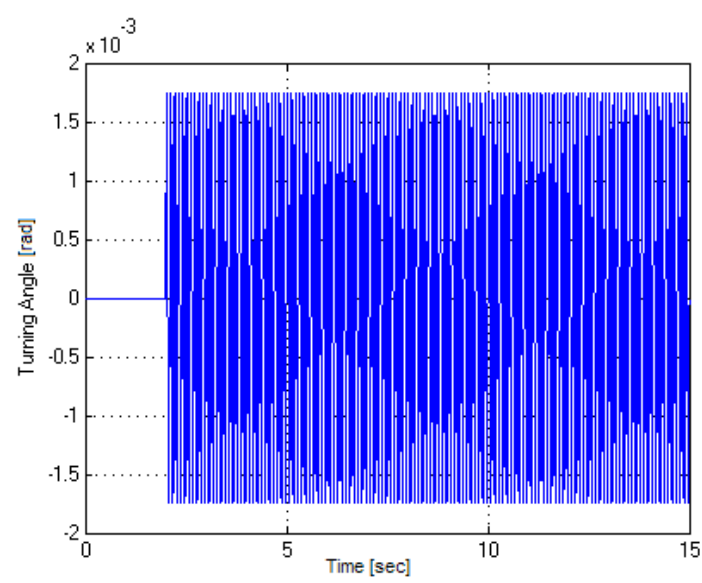

Figure 64. Turning angle generated by shimmy effects.

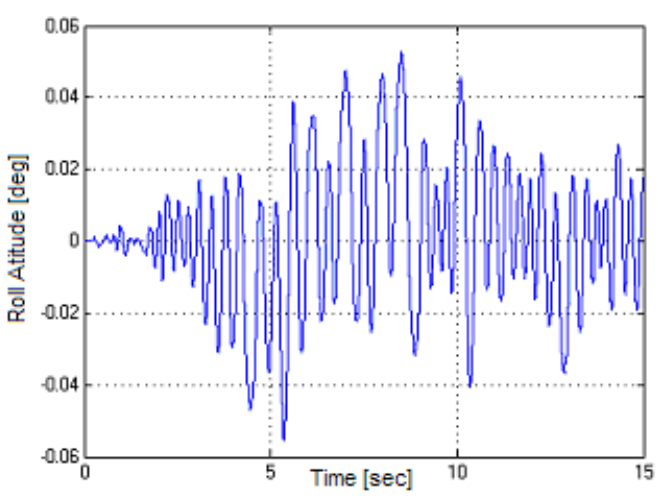

Figure 65. Roll attitude generated by shimmy effects.

\section{Non-Deploy:}

This last part of this section deals with the simulation of the aircraft when the simulator user forgets to engage the landing gear or it snaps off. Failures of this type are the most prevalent. Unfortunately, they are also the most difficult to model. In Figure 66 , the largest change in the aircraft states is shown in the pitch attitude being lowered to $-16^{\circ}$. This is caused by the third balancing point of the aircraft being the fuselage of the aircraft. With the angle of the aircraft being lowered, the altitude of the $\mathrm{CG}$ is in turn lowered as is seen in Figure 67. Since the aircraft is now being pushed along the landing surface with possible structural destructions instead of rolled as is the standard case, the acceleration of the aircraft drops dramatically to $.4 \mathrm{~m} / \mathrm{s}^{2}$ as can be derived from Figure 68 . As noted in the tire loss section the forward pitching of the aircraft causes the load on the nose to be increased. In Figure 69 it can be seen that the load is increased to $12,000 \mathrm{~N}$. This is roughly three times the original force supplied by the nose under normal 
conditions. The short time period where there is no force generated by the landing gear occurs when the strut changes from being extended at two seconds until it falls to the landing surface. It can be seen that the dynamics of the landing surface force is a second order response. This correlates to the assumption that the surface is modeled as a rigid spring and damper system. With this model, two oscillations occur in two seconds with a maximum oscillation of .3 degrees of pitch attitude and $7 \mathrm{~cm}$ of altitude.

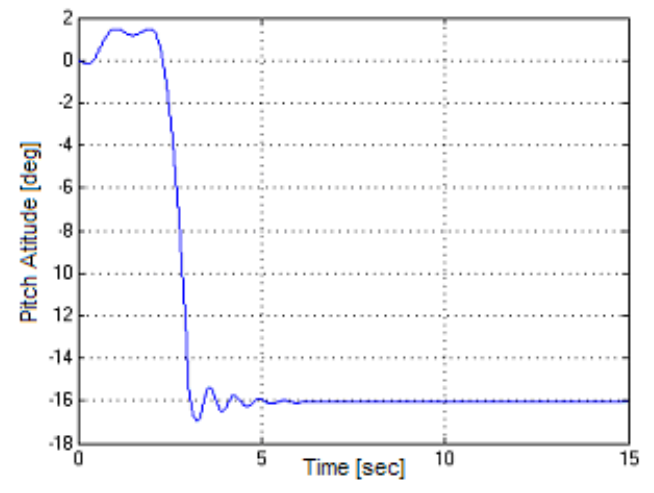

Figure 66. Pitch attitude when the nose gear is not deployed.

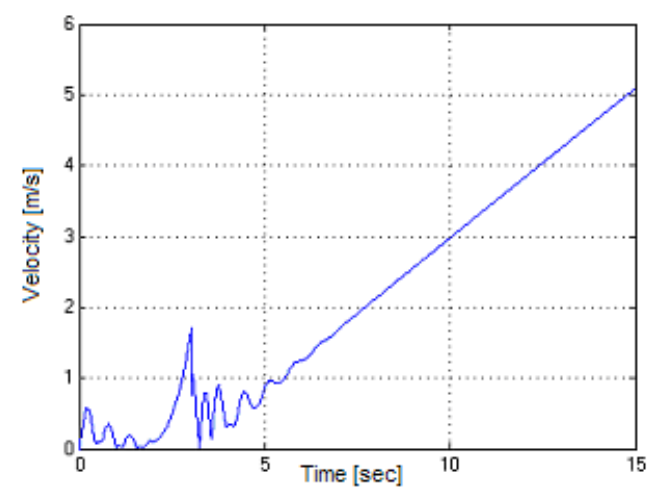

Figure 68. Velocity when the nose gear is not deployed.

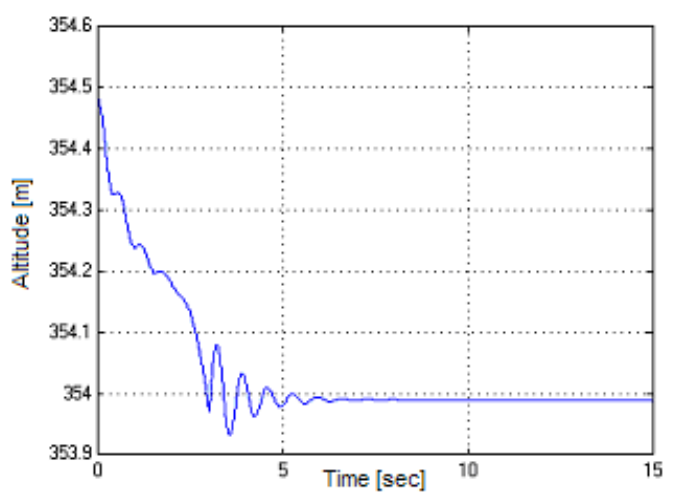

Figure 67. Altitude when the nose gear is not deployed.

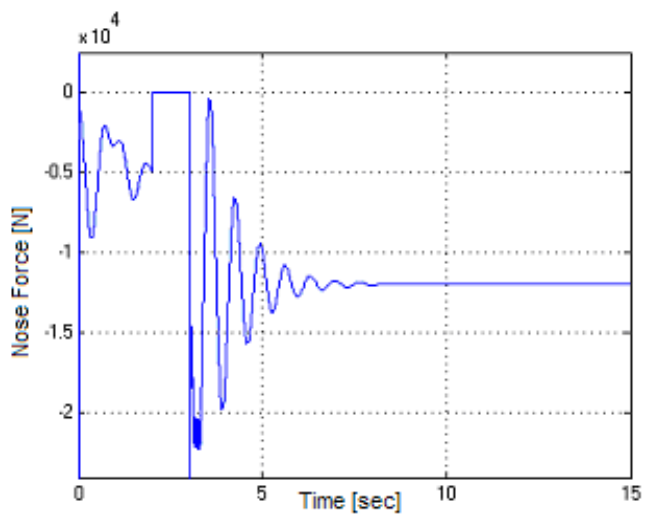

Figure 69. Nose force when the nose gear is not deployed 


\section{B3. Moving Tests (With Brakes): \\ Brake Degradation:}

For the simulation of this failure, the left braking capability was cut in half. This causes two events to occur. First, the aircraft will yaw towards the direction with the higher braking capability. Since a positive rotation is associated with a yawing to the right, Figure 70 shows the expected results of an aircrafts left brake degrading. Figure 71 gives the braking force available for use in the friction algorithm stipulated above. The max force after the failure around $3000 \mathrm{~N}$ is not half of the normal braking force since friction is included in the depicted force. This test acts opposite to as if one of the brakes were jammed. Instead of yawing away from the side with the failure, it would instead yaw towards it.

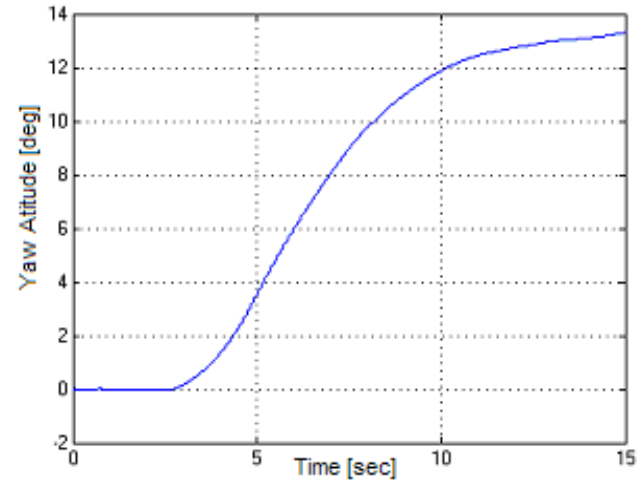

Figure 70. Yaw produced with left brake degradation.

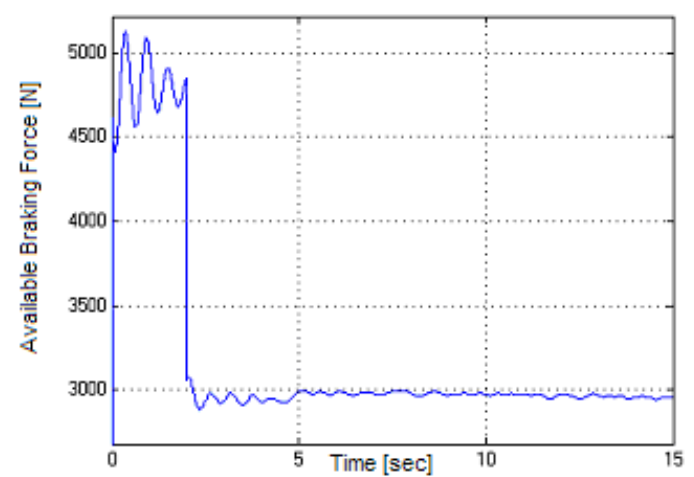

Figure 71. Available braking force with left brake degradation.

\section{B4. Designer Usage Demonstration:}

To display the capabilities of the Designer Usage portion of the flight simulator, a small experiment was conducted. Even though the dynamic parameters passed a qualitative assessment of the landing gear system given by pilots accustomed to this aircraft, all of the dynamic parameters were adjusted over a range. By doing this, the 
effect each component has on the states can be viewed. The main components were changed in conjunction with each other. For this test, the aircraft was placed on the runway with the wheels just in contact with the surface of the runway with no vertical or horizontal velocity. The black dotted line is the test conducted with higher values for that parameter. Red dashed lines correspond to tests where the parameter value was reduced. The unaltered response is given by the solid blue line. Except when noted, the tests have a twenty-five percent increase in the parameter for the black line and a twenty-five percent reduction for the red line. The one thing that held true for all of the tests was that changing the dynamic parameter caused a non-linear transformation on the response.

\section{Main Strut Spring:}

As can be seen in Figure 72 , the altitude of the aircraft drops nearly $15 \mathrm{~cm}$ with a decrease in the spring coefficient. This is due to the lower spring coefficient needing farther to deflect. Figure 73 shows a large increase in pitch with the lowering of the main spring coefficient. Between the two graphs, it seems that altering the main strut spring component has a proportional effect on altitude and an inverse effect on the pitch. Force in the struts and the tires are not greatly affected by the change in the strut spring coefficient as seen in Figure 74 and 75. A slight increase in the overshoot of the forces can be detected with a larger spring coefficient. Lowering the spring coefficient causes a small decrease in natural frequency. 


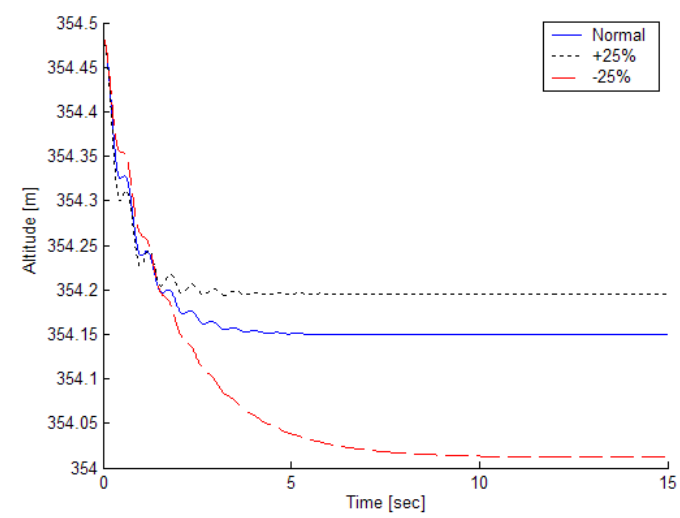

Figure 72. Altitude when subjected

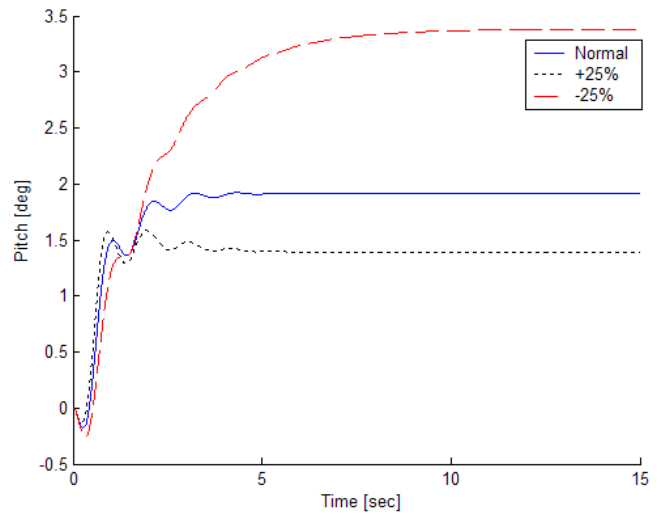

Figure 73. Pitch attitude when subjected to a main oleo spring degradation. to a main oleo spring degradation.
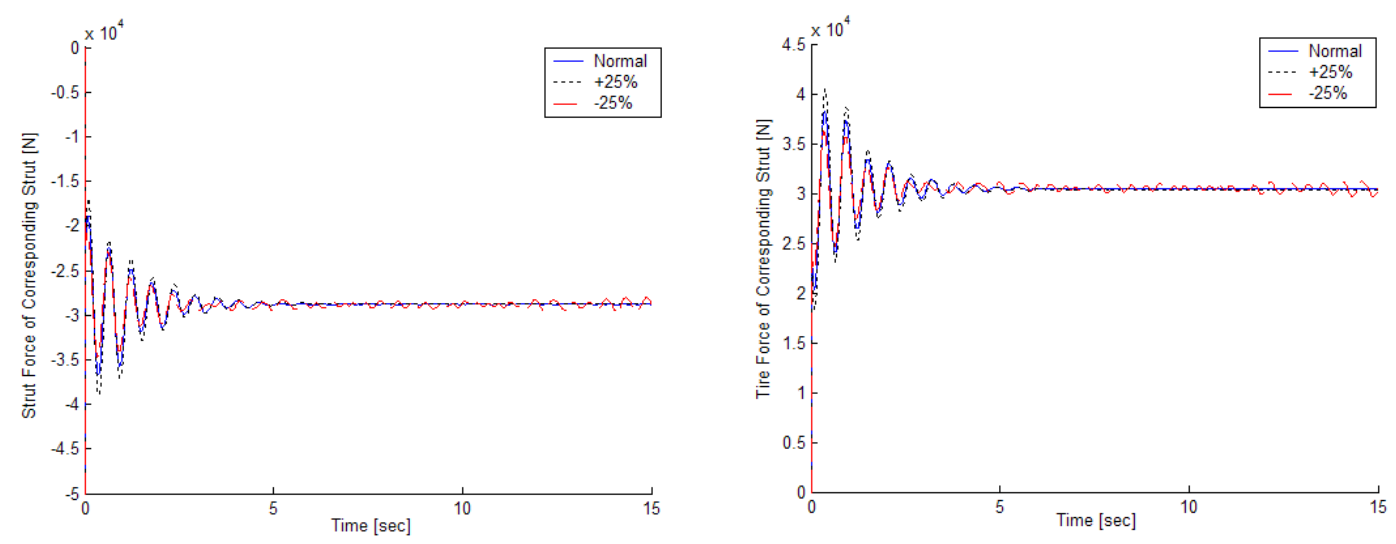

Figure 74. Main strut force when subjected Figure 75. Main tire force when subjected to a main oleo spring degradation. to a main oleo spring degradation.

\section{Main Tire Spring:}

The same effect can be seen in Figures 76 and 77 on the steady state conditions of the altitude and pitch as was seen in the prior subsection. A lower main tire spring coefficient lowers the altitude and increases the pitch. An increase in amplitude of the 
oscillations can also be seen with a lower value. Unlike the prior subsection, making alterations to the tire stiffness has a quite visible impact on the strut and tire forces. In both Figures 78 and 79, it can be seen that lowering the stiffness causes the overshoot to jump drastically. Settling time is also increased substantially. This in turn implies that the natural frequency is decreased.

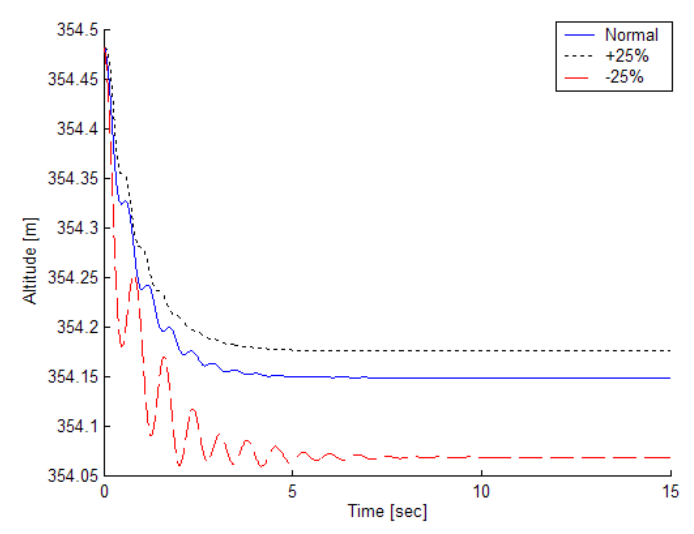

Figure 76. Altitude when subjected to a main tire spring degradation.

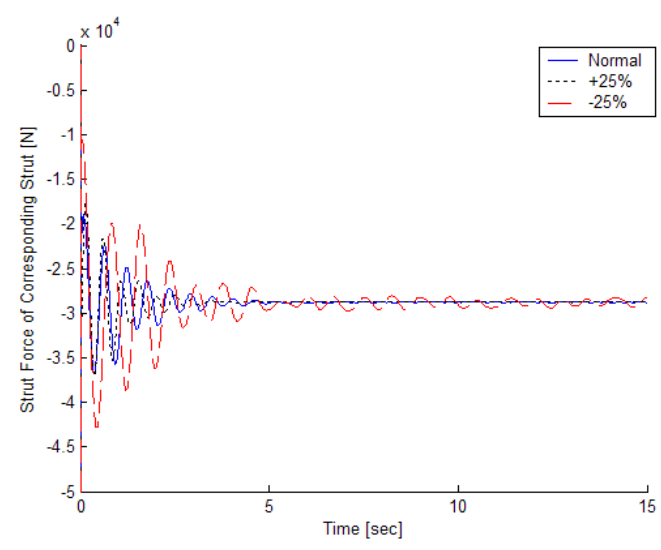

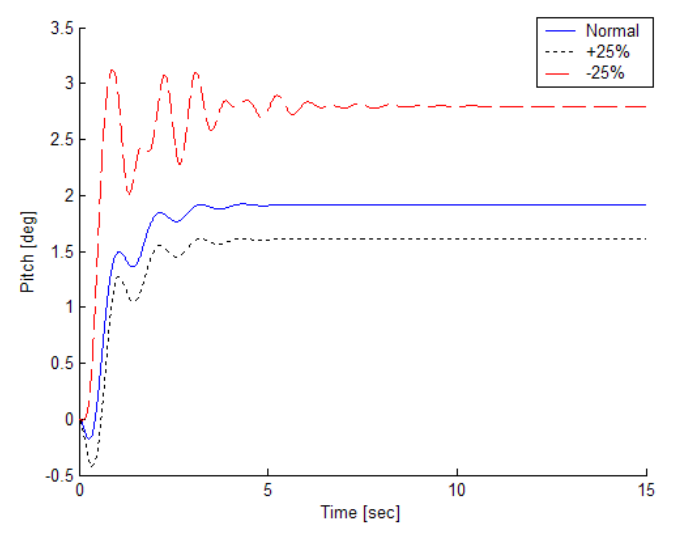

Figure 77. Pitch attitude when subjected to a main tire spring degradation.

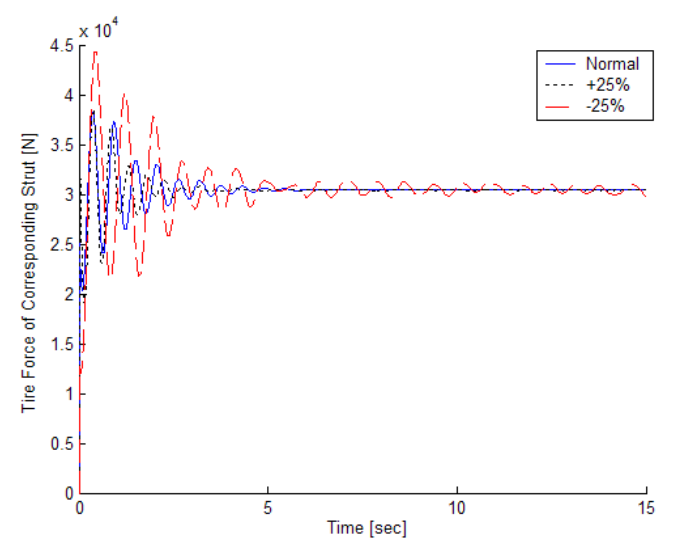

Figure 78. Main strut force when subjected Figure 79. Main tire force when subjected to a main tire spring degradation. to a main tire spring degradation. 


\section{Main Strut Damper:}

Raising the damper coefficient causes the settling time to be increased. This can be seen in both Figures 80 and 81 . Closely examining the corresponding strut and tire force graphs, Figure 82 and 83, an odd occurrence can be viewed. Initially, the lower damping value has a higher overshoot, but before the system comes to steady state the high damping value has a higher overshoot. Since this is a multiple degree of freedom system, this change in overshoot could be the result of another slower dynamic. Figures 84 and 85 show the expected lower overshoot values but on the other landing gear strut.

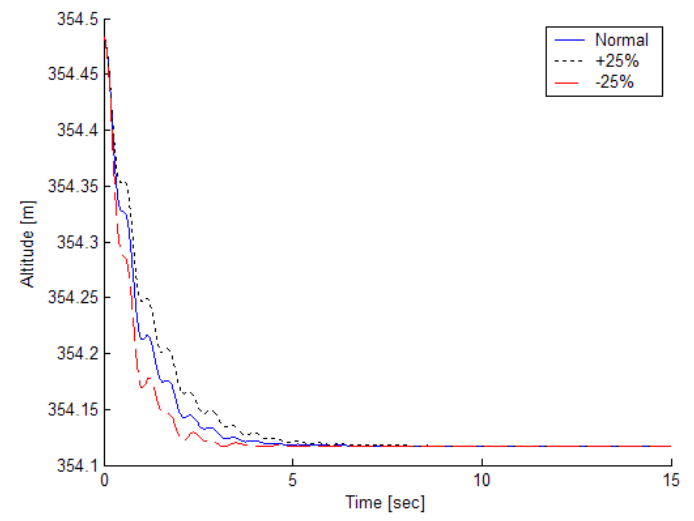

Figure 80. Altitude when subjected to a main oleo damper degradation.

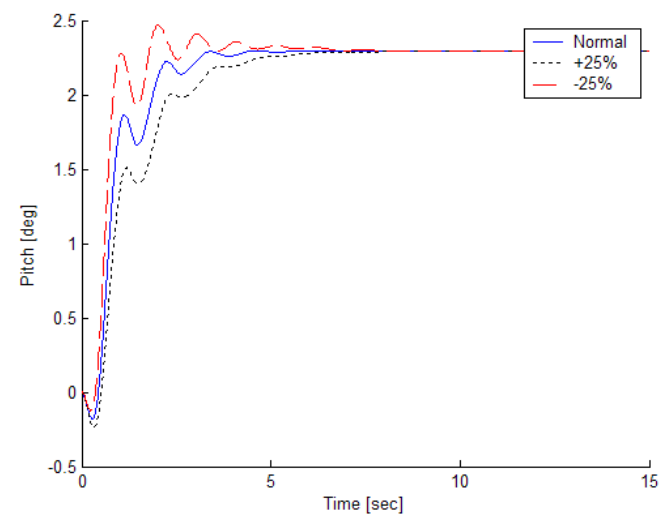

Figure 81. Pitch attitude when subjected to a main oleo damper degradation. 

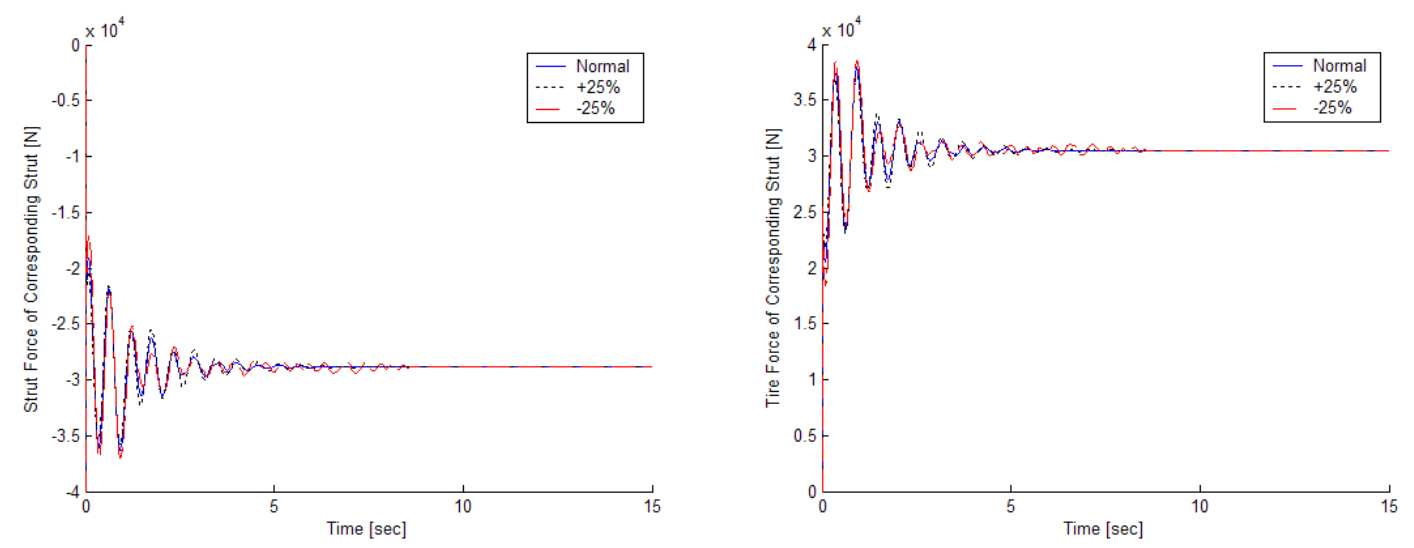

Figure 82. Main strut force when subjected Figure 83. Main tire force when subjected to a main oleo damper degradation. to a main oleo damper degradation.
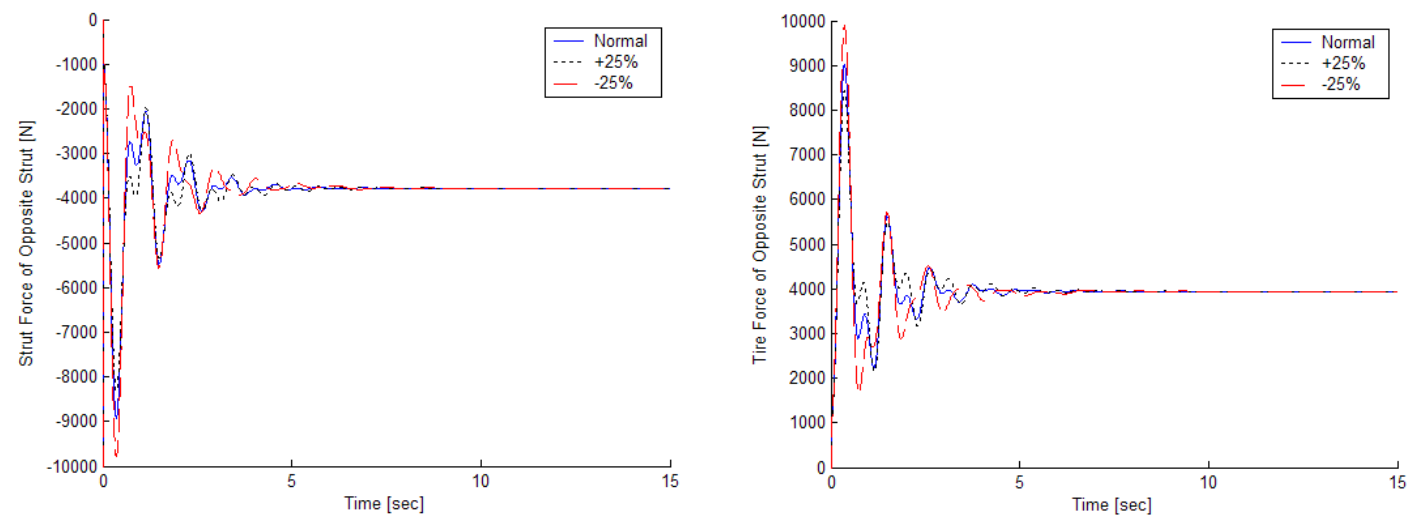

Figure 84. Nose strut force when subjected Figure 85. Nose tire force when subjected to a main oleo damper degradation. to a main oleo damper degradation.

\section{Nose Strut Spring:}

Unlike the main strut spring test, altering the nose strut spring has very little effect on changing the altitude of the aircraft as seen in Figure 86. This is due to the long distance that the CG is away from the nose strut as compared to the main struts. Figure 87 , however, shows results very similar to the previously mentioned part. The largest 
difference is that lowering the nose spring coefficient lowers the aircrafts pitch. This is due to it being fore of the CG instead of behind it. The force in the strut and tire is, for the most part, unaffected by changing the strut spring coefficient as seen in Figure 88 and 89. The change in steady state could be from the pitching of the aircraft.

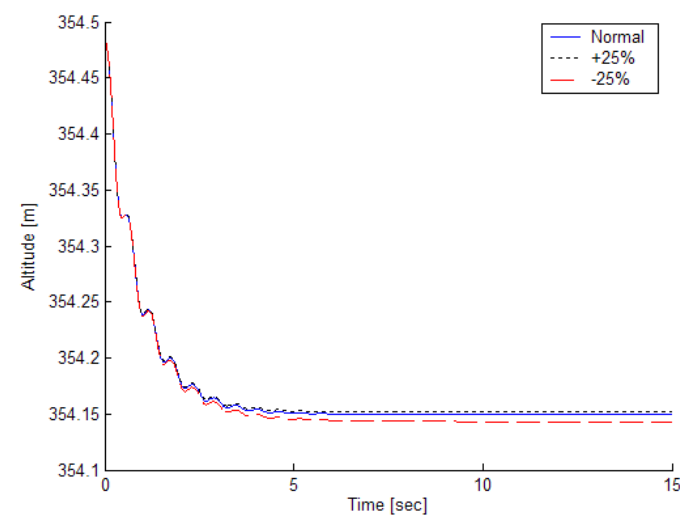

Figure 86. Altitude when subjected to a nose oleo spring degradation.

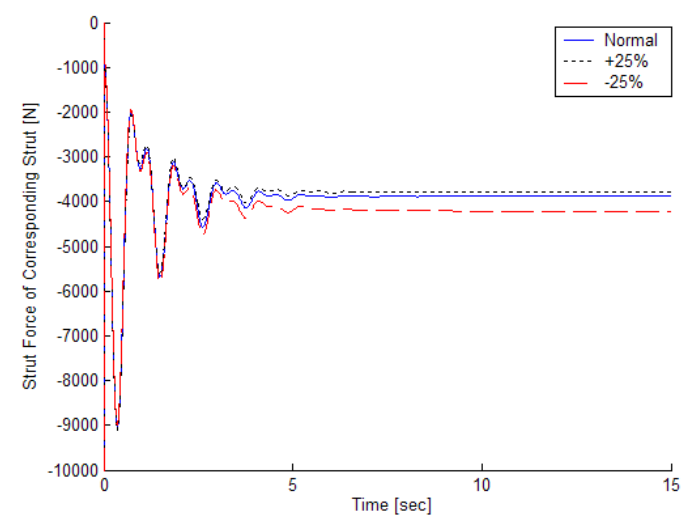

Figure 88. Nose strut force when subjected to a nose oleo spring degradation.

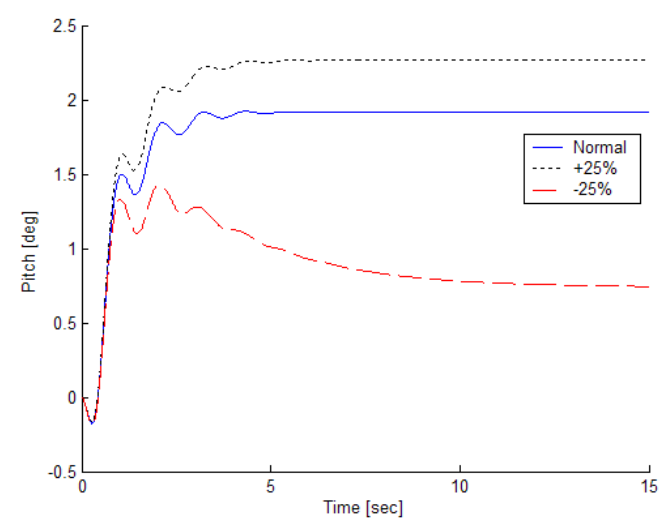

Figure 87. Pitch attitude when subjected to a nose oleo spring degradation.

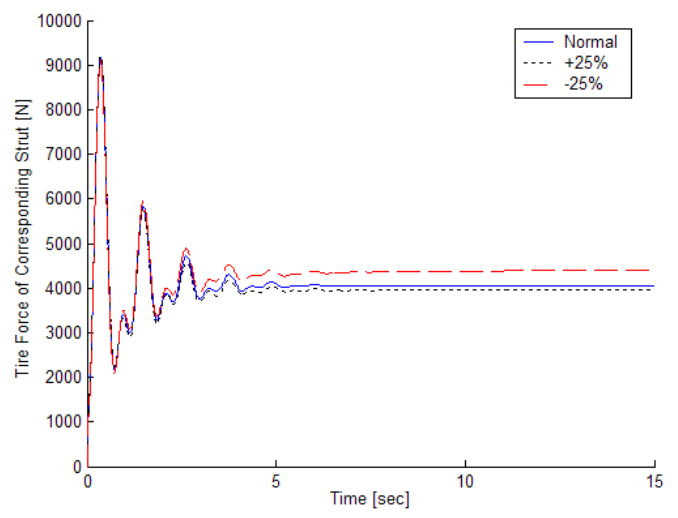

Figure 89. Nose tire force when subjected to a nose oleo spring degradation. 


\section{Nose Tire Spring:}

After discussing the main spring responses compared to one another, the responses of the nose wheel tire are no surprise. As before, the altitude and pitch response pictured in Figure 90 and 91 resemble that of the corresponding strut spring. The force results are a different story however. In Figures 92 and 93, it can be seen that lowering the tire spring coefficient causes one of the modes of the response to be removed. This is most likely due to the hub of the wheel coming into contact with the landing surface.

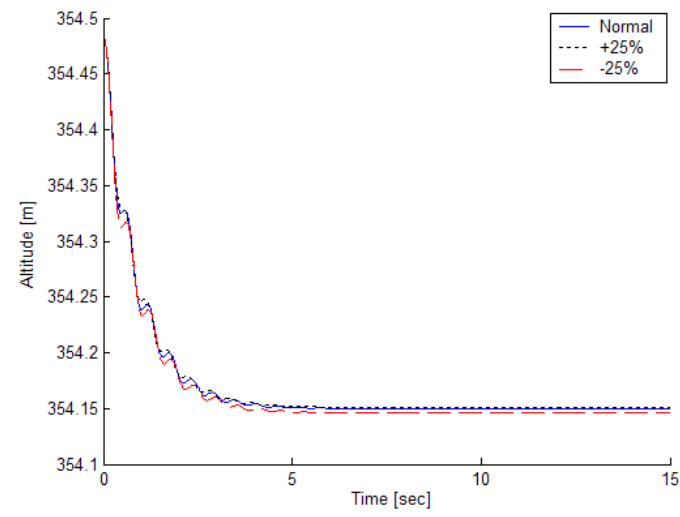

Figure 90. Altitude when subjected to a nose tire spring degradation.

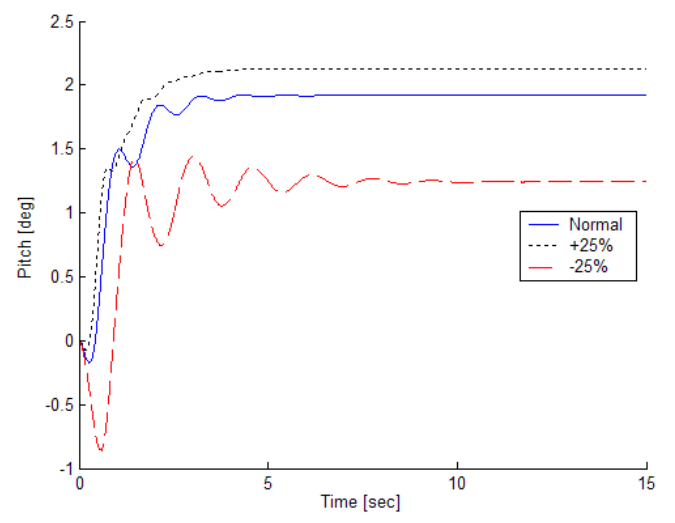

Figure 91. Pitch attitude when subjected to a nose tire spring degradation. 

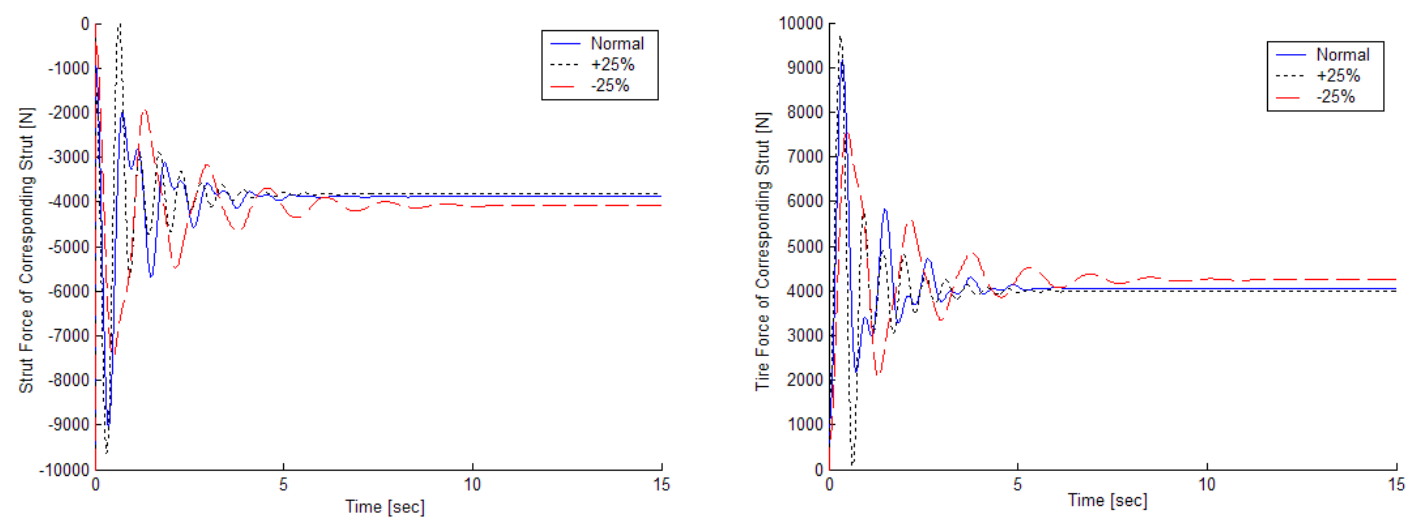

Figure 92. Nose strut force when subjected Figure 93. Nose tire force when subjected to a nose tire spring degradation. to a nose tire spring degradation.

\section{Nose Strut Damper:}

To perform this test, the testing conditions were altered. For this test, the black dotted line is the normal response, the solid blue line a twenty-five percent reduction, and the dashed green line a fifty percent reduction. This change was done because this component corresponds to the fastest mode and raising its value necessitates a decrease in calculation step size. As was the case with all of the nose components, the altitude response is mostly untouched as seen in Figure 94. This is most likely due to the fast dynamic mode that this component is related too. Pitch, too, is barely affected by changing the damping coefficient. This is displayed in Figure 95. The higher damping coefficient causes a lower overshoot pictured in Figure 96 and 97 . The greater steady state force could be from the aircraft being pitched downward more, putting a greater load on the nose. The force in the main landing struts is largely unchanged as can be seen in Figures 98 and 99. 


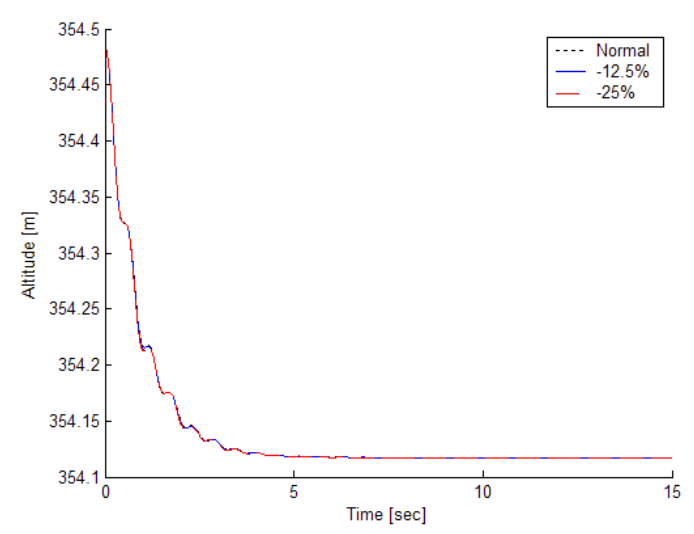

Figure 94. Altitude when subjected

to a nose oleo damper degradation.

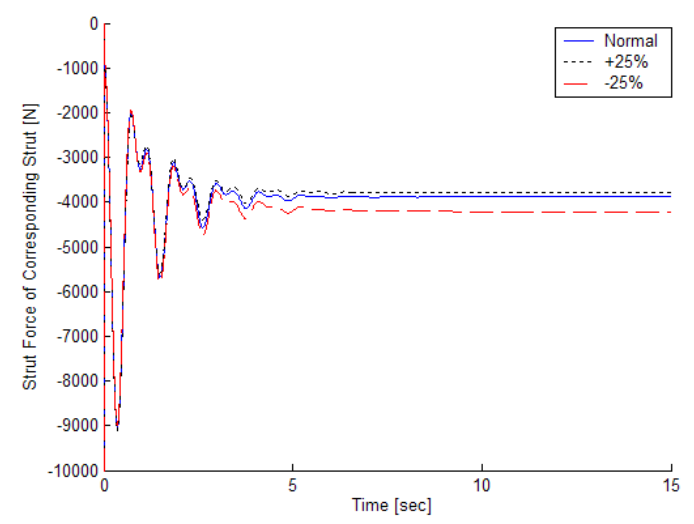

Figure 96. Nose strut force when subjected Figure 97. Nose tire force when subjected to a nose oleo damper degradation.

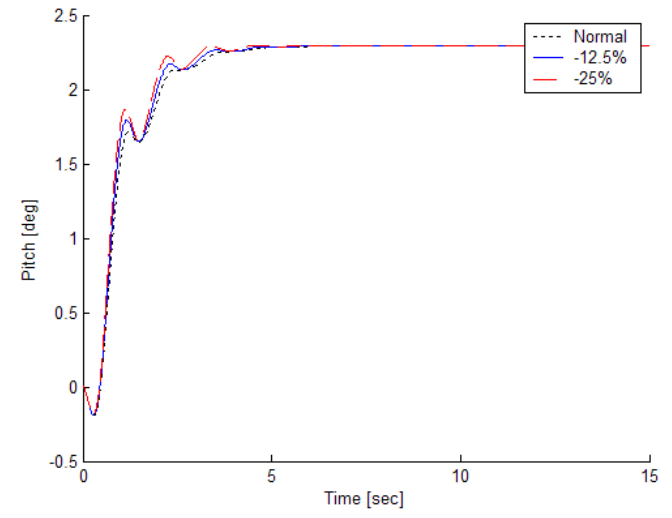

Figure 95. Pitch attitude when subjected

to a nose oleo damper degradation.

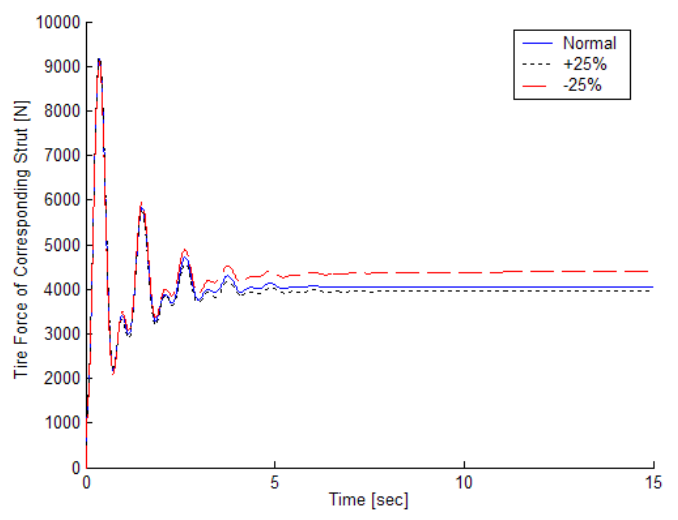

to a nose oleo damper degradation. 


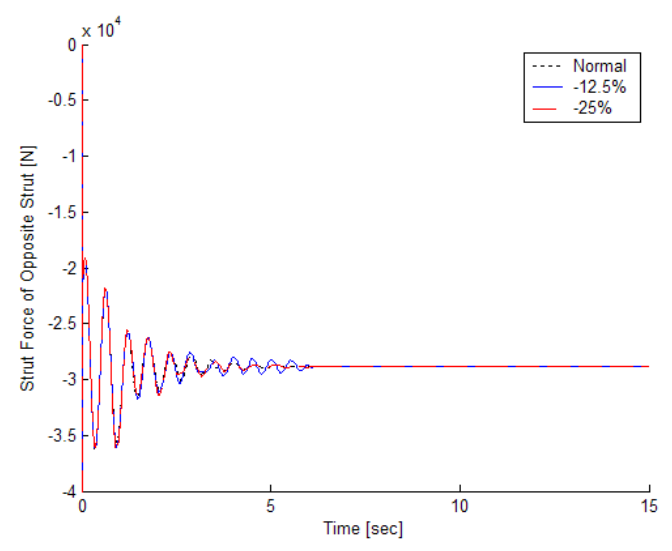

Figure 98. Main strut force when subjected to a nose oleo damper degradation.

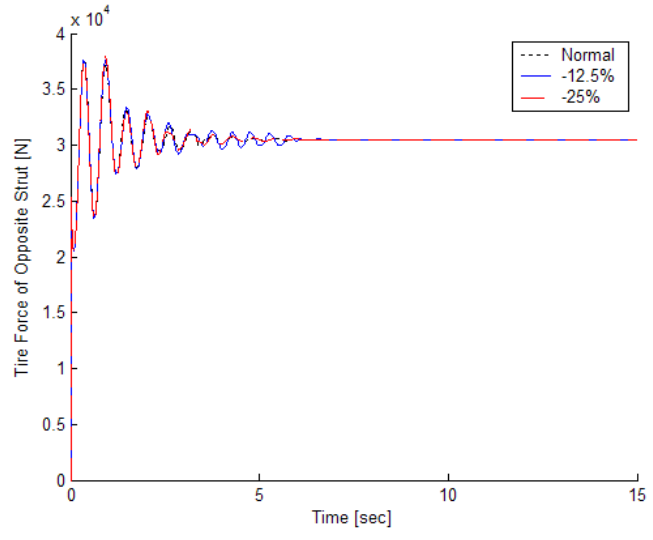

Figure 99. Main tire force when subjected to a nose oleo damper degradation. 


\section{Conclusions and Recommendations}

A simulator that modeled failures while on the landing surface was created. It was also shown that the simulator can be used to test the dynamic response of an aircraft upon landing. With all of the results from simulator testing coming as expected, the simulator is applicable in an academic aspect. Additionally, the alteration of the dynamic responses created by changing parts of the landing gear system can be viewed. With the failure of degradation of strut components it is seen that the user has the ability to test different component values, showing that there is a design option to the simulator. The simulator can be used as a design aide, for an academic tool, or an interfacing tool with a training device. These proceeding statements lend to the objectives as having been met.

There are several ways that this model can be improved. The most obvious way to do this would be to negate making any of the assumptions that were made to lower the complexity of the model. The dynamic coefficients of the landing gear could be better mapped along its entire deflection range instead of using a mean value. A way to model downdraft and thermals would also expand the simulator. Development of an algorithm to define the strut deflection line to more accurately reflect the real world deflection of the landing gear would make the model more accurate. Lastly, the model of the landing surface could be advanced to allow for a dynamic rough surface to allow the landing of real-world airports. The biggest recommendation to updating the model would be to have failure data to test against. Gaining this data could be difficult due to the proprietary nature of this data. Increasing the accuracy of the model would have to be done for each aircraft to be modeled. 


\section{Bibliography}

[1]. "Shock Absorber." Wikipedia. Wikimedia Foundation, Inc, 2010. Web. 27 May 2010.

[2]. "Flight Simulator." Wikipedia. Wikimedia Foundation, Inc, 2010. Web. 7 Jun 2010.

[3]. "Link Trainer." Wikipedia. Wikimedia Foundation, Inc, 2010. Web. 4 May 2010.

[4]. "Service Bulletin 95." Glasair Aviation. Stoddard-Hamilton, 19 Feb 1990. Web. 7 Jun 2010. <http://www.glasairaviation.com/pdf/GSB095.PDF>.

[5]. Boser, Robert J. "JetBlue Emergency Landing: LAX 050921." AirlineSafety.com. 21 Sep 2005. Web. 7 Jun 2010. <http://www.airlinesafety.com/editorials/JetBlueLAX.htm>.

[6]. "Aircraft Model Has History of Landing-Gear Problems." Fox News. 13 Feb 2009. Web. 7 Jun 2010.

<http://www.foxnews.com/story/0,2933,492325,00.html>.

[7]. Niebuhr, David. "Aviation Failure.” Metallurgy Consultant. Niebuhr

Metallurgical Consulting. Web. 7 June 2010.

$<$ http://metallurgyconsultant.com/aviation-failure.php>.

[8]. “Undercarriage." Wikipedia. Wikimedia Foundation, Inc, 2010. Web. 31 May 2010.

[9]. "Proposed Rules." Federal Register. 72, No. 203. FAA, 2007. Print.

[10]. Banks, Carl. "A paper in Aerospace Engineering." A Discussion of Methods of Real-Time Airplane Flight Simulation. The Pennsylvania State University, 2000. Print.

[11]. Lindsley, Ned J. "A Tire Model for Air Vehicle Landing Gear Dynamics." 2000 International ADAMS User Conference. (2000): Print.

[12]. Lernbeiss, R. "Simulation Model of an Aircraft Landing Gear Considering Elastic Properties of the Shock Absorber." Journal of Multi-body Dynamics. 221. (2007): 77-86. Print.

[13]. Pritchard, Jocelyn. "Overview of Landing Gear Dynamics." Journal of Aircraft. 38.1 (2001): 130-7. Print.

[14]. Ghiringhelli, Gian. "Testing of Semiactive Landing Gear Control for a General Aviation Aircraft." Journal of Aircraft. 37.4. (2000): 606-16. Print.

[15]. De Yoe, Ronald A.. "Thesis." Active Control for Aircraft Landing Gear. Air Force Institute of Technology, 1970. Print.

[16]. Mikulowski, Grzegorz. "Adaptive Landing Gear: Optimum Control Strategy and Potential for Improvement." Shock and Vibration 16. (2009): 175-94. Print.

[17]. Sura, Niranjan K. "Lateral Stability of Aircraft Nose-Wheel Landing Gear with Closed-Loop Shimmy Damper.” Journal of Aircraft. 46.2 (2009): 505-9. Print.

[18]. Sateesh, B. "The Anaylsis of Shimmy Instability of a Typical Nose Landing Gear Using Active Torsional Magneto-Rheological Damper." JAERO437. 223.G (2009): 201-16. Print.

[19]. Fallah, M. S. "Robust Model Predictive Control of Shimmy Vibration in Aircraft Landing Gear.” Journal of Aircraft. 45.6 (2008): 1872-1880. Print. 
[20]. Sura, Niranjan K. "Closed-Form Analytical Solution for the Shimmy Instability of Nose-Wheel Landing Gears.” Journal of Aircraft. 44.6 (2007): 1985-90 Print.

[21]. Verzichelli, Gianluca. "Development of an Aircraft and Landing Gear Model with Steeting System in Modelica." Modelica 2008. 2008. Filton, United Kingdom: The Modelica Association, 2008. Print.

[22]. Denery, Terry. "Creating Flight Simulator Landing Gear Models Using Multidomain Modeling Tools." Natick, MA: The MathWorks, Inc., 2006. Print.

[23]. Kane, Thomas R. Dynamics: Theory and Applications. 1st. McGraw-Hill, Inc., 1985. 1-14. Print.

[24]. Dreier, Mark E. Introduction to Helicopter and Tiltrotor Simulation. $1^{\text {st }}$ ed. Reston, VA: American Institute of Aeronautics and Astronautics, Inc., 2007. 32351. Print.

[25]. York, Brent. “A Physically Representative Aircraft Landing Gear Model for RealTime Simulation." Naval Air Systems Command. Patuxent River, MD: Department of the Navy, 1996. Print.

[26]. Roskam, Jan. Airplane Flight Dynamics and Automatic Flight Controls. $4^{\text {th }}$ Printing. 1. Lawrence, KS: DARcorporation, 2003. 3-32. Print.

[27]. Ogata, Katsuhiko. Modern Control Engineering. $4^{\text {th }}$ ed. Upper Saddle River, NJ: Prentice Hall, 2002. 85-90. Print.

[28]. "Reference Tables -- Coefficient of Friction." Engineer's Handbook. EngineersHandbook.com, 2006. Web. 7 Jun 2010. <http://www.engineershandbook.com/Tables/frictioncoefficients.htm>.

[29]. Hibbeler, R.C. Engineering Mechanics: Dynamics. $10^{\text {th }}$ ed. Upper Saddle River, NJ: Prentice Hall, 2004. 321. Print.

[30]. Edward, C. Henry. Calculus: Early Transcendentals Version. $6^{\text {th }}$ ed. Upper Saddle River, NJ: Prentice Hall, 2003. 798. Print.

[31]. Ossa, E.A. "Failure Analysis of a Civil Aircraft Landing Gear." Engineering Failure Analysis 13. (2006): 1177-83. Print.

[32]. Currey, Norman S. Aircraft Landing Gear Design Principles and Practices. $1^{\text {st }}$ ed. Washington D.C.: American Institute of Aeronautics and Astronautics, Print. 


\section{Appendix}

\section{User Manual}

\section{Purpose}

The goals of this simulator are oriented at creating tools for the design and analysis of fault tolerant control laws, landing gear development, and failure simulation in an academic setting. The user will be able to simulate flying a business jet aircraft that can experience abnormal conditions in the way of failures. These failures can either be picked by an observer or generated by the pilot themselves. Additionally, the simulator can be used to generate a response for a virtually created aircraft to view the dynamic response of the landing gear.

\section{Designer Aide}

System Requirements: $\quad$ MatLab Version 6.5.0.180913a (R13)

Simulink Version 5.0 (R13)

Additional Toolboxes: RTB, Real-Time Constraints

SMXL, Vector Calculations

Dequiv, GUI Input Devices

Operating System: Requirements Given by MatLab

Processor: $\quad$ Requirements Given by MatLab

As Advanced as Possible (Recommended)

Memory: $\quad 3 \mathrm{MB}$

Video: $\quad$ Requirements Given by MatLab

Sound: $\quad$ Requirements Given by MatLab 


\section{Failure Simulator}

System Requirements: X-Plane Version 7

Operating System: $\quad$ Requirements Given by X-Plane

Processor: $\quad$ Requirements Given by X-Plane

Video: $\quad$ Requirements Given by X-Plane

Sound: $\quad$ Requirements Given by X-Plane

Installation Instructions

Copy the folder 'LandingGear_Current' into the C:|Work_Files\Matlab_Files $\backslash$ directory. Ensure that the additional toolboxes are a part of the MatLab path.

\section{Troubleshooting}

If any trouble is experienced while running the landing surface failure simulator, refer to Modeling and Simulation of Tricycle Landing Gear at Normal and Abnormal Conditions. This paper explains how the model was created, the flow of the GUIs and what the Simulink blocks do. If the issue is due to a possible singularity in the solution, decrease the value of $\mathrm{T}$ in the MatLab command window. Due to integration issues, the error may be caused by using a newer version of MatLab.

\section{Technical Support Contacts}

To contact the creator of this program, e-mail them at Snave.Lihp@gmail.com with any questions or problems. Typically a response will be given within 72 hours.

\section{Simulation Performance}

If slow or choppy flight simulation is encountered, computational capabilities of the computer are inefficient. Contact technical support for a way to alleviate this 
situation at the expense of modeling accuracy. The best option is to replace or update all hardware.

\section{Using the Simulator}

To start the simulator program, enter LandSimFig into the MatLab command window while in the LandingGear_Current directory. This brings up the GUI presented in Figure 1. As can be seen, this figure has only two options. The first choice is to use the simulator as a pseudo-pilot trainer. It is not a real pilot trainer since validation data is a rarity. The second option is to use the simulator to aide in the development stage of landing gear design. This allows designers to create a virtual aircraft that can be used to test the dynamics of the landing gear system.

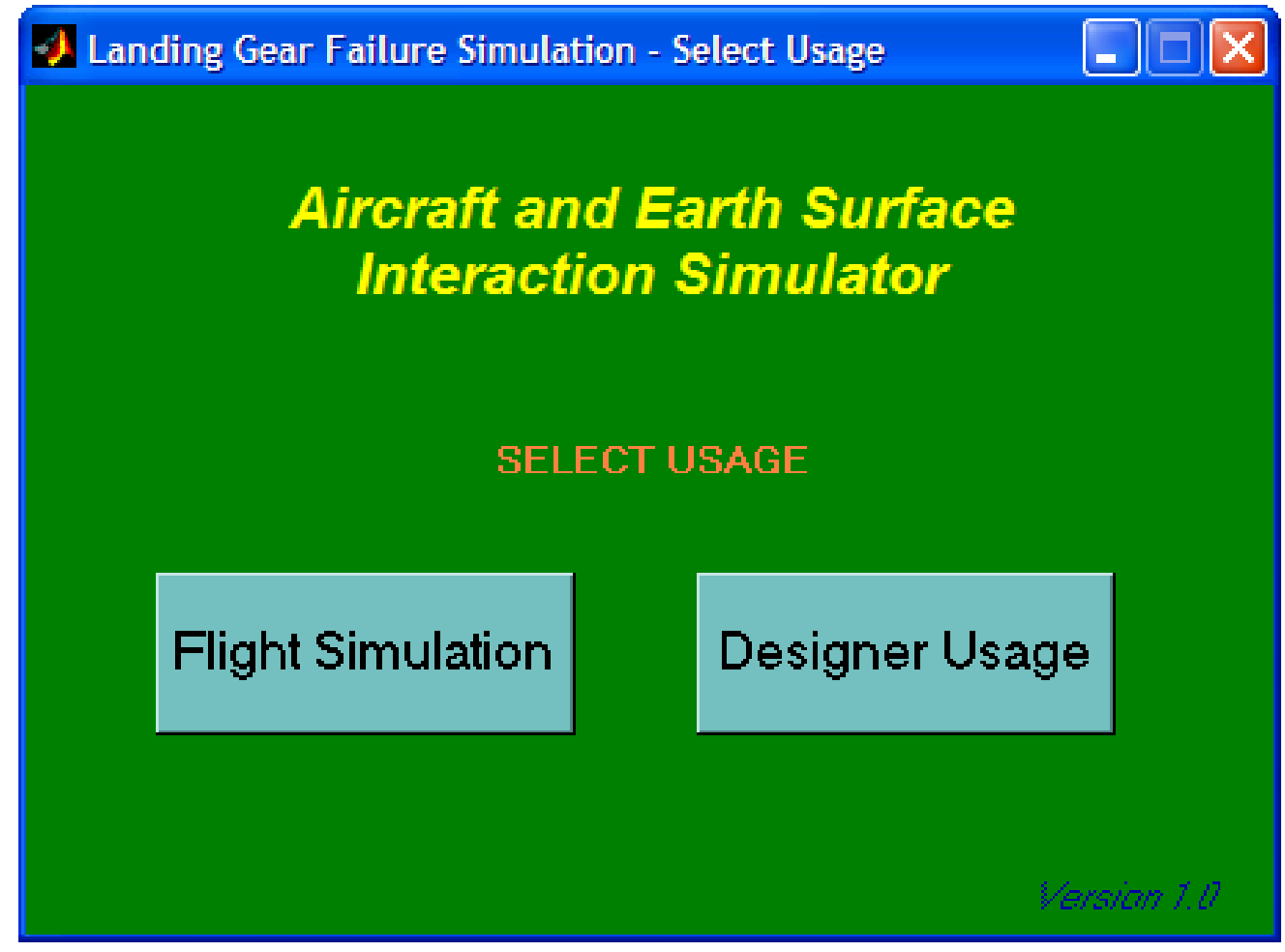

Figure 1. Initial GUI of the Landing Failure Simulator.

Clicking on the Pilot Training button brings up Figure 2. In this GUI, the choice of how the failure is to be initialized must be made. The options are between whether to 
have the failure triggers chosen prior to the aircraft flight being simulated or during it. The first option allows for the same test to be ran repetitively. Real time failure injections are useful for observers to determine the capabilities of the pilot.

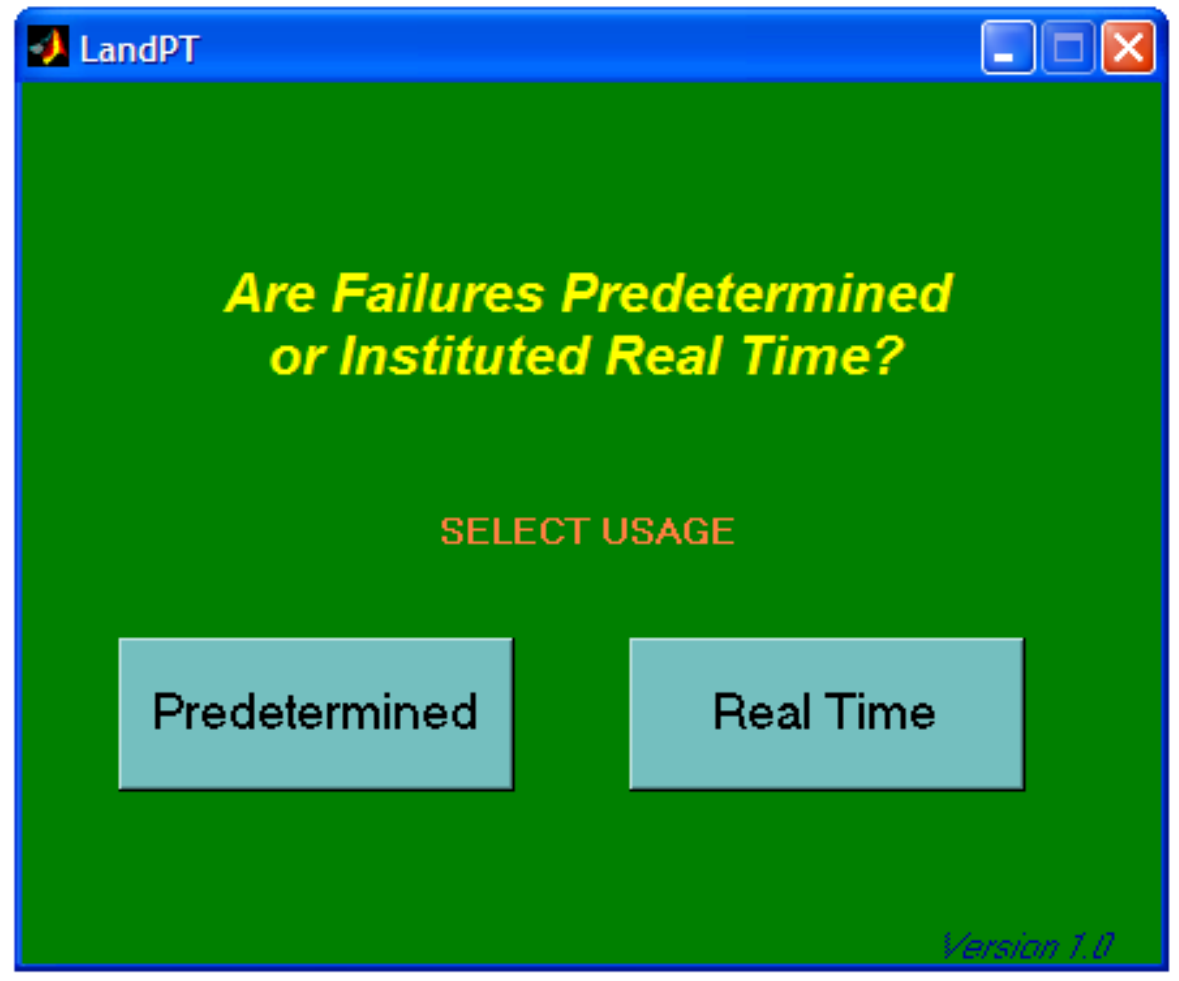

Figure 2. Selecting the Failure Initialization Type.

Predetermined failures summon Figure 3 for the user to view. This window is where the location of the failure must be chosen. A failure can occur on the nose, the left main or the right main struts. Additionally, the simulator can be set to run with no set failure. These failures are the same for each strut with the exception of shimmy and brake jamming. Only the nose strut can experience shimmy since it is the only strut capable of torsion rotation. Brake jamming only occurs on the main landing gear because these struts are the only ones with brakes on them. Brakes are not included on the nose strut to minimize the possibility of flipping the plane. 


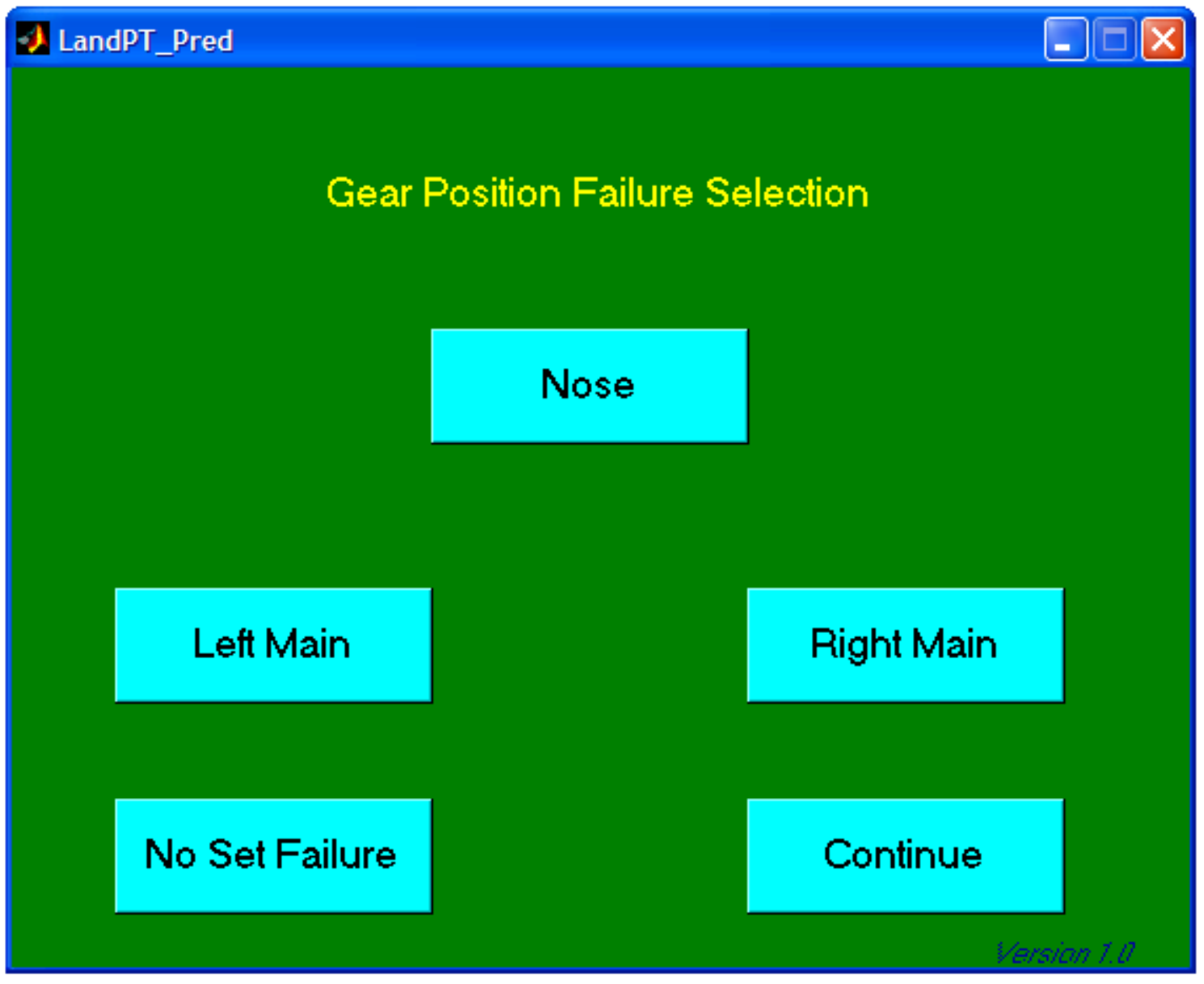

Figure 3. Selecting the Failure Location.

Since the failure selection screens are very similar, only the nose failure selection window will be shown in Figure 4. As can be seen, there are seven possible failures on the nose landing gear strut. Whenever a failure is selected, the Load Failure push button is revealed. Selecting a failure also has another effect; it hides the impossible failure options. Jamming a strut implies that the Strut's spring and damper would have no effect, therefore they would be removed from the list of possible failures. Tire Loss, Flat Tire, and Tire Blowout Torsion all have a similar negating relationship. The sliders represent a range of severity of the failure that they are across from. The Return to Position Selection push button returns the user to the previous menu. 


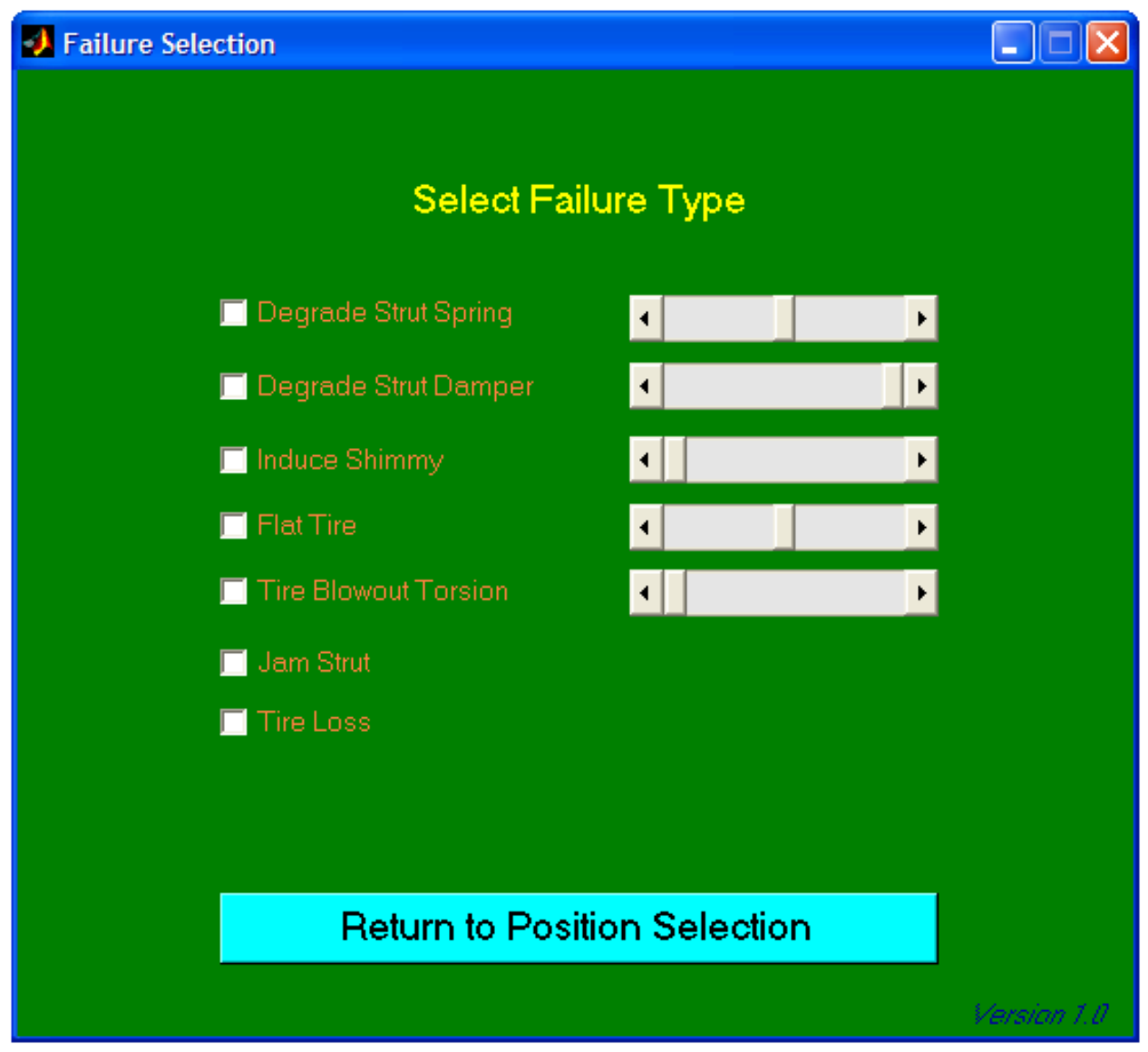

Figure 4. Selecting the Failure Type of the Nose Wheel.

After choosing a failure, the triggering scenario must be picked from the window depicted in Figure 5. The triggering options are either to have the aircraft fail on initialization or after a certain amount of time has elapsed after coming into contact with the landing surface. Having the failure trigger on initialization models the strut failing during deployment/undeployment if the aircraft starts in the air or poor pre-flight checks if the aircraft starts on the surface. The landing system failing after coming into contact with the ground models the vehicle failing from stress or fatigue. To advance this option, there is an additional option where the user can limit a threshold on the force in the tire and the oleo. Surpassing these limits cause the tire to flatten or the oleo to jam or disconnect depending on which threshold level is passed. 


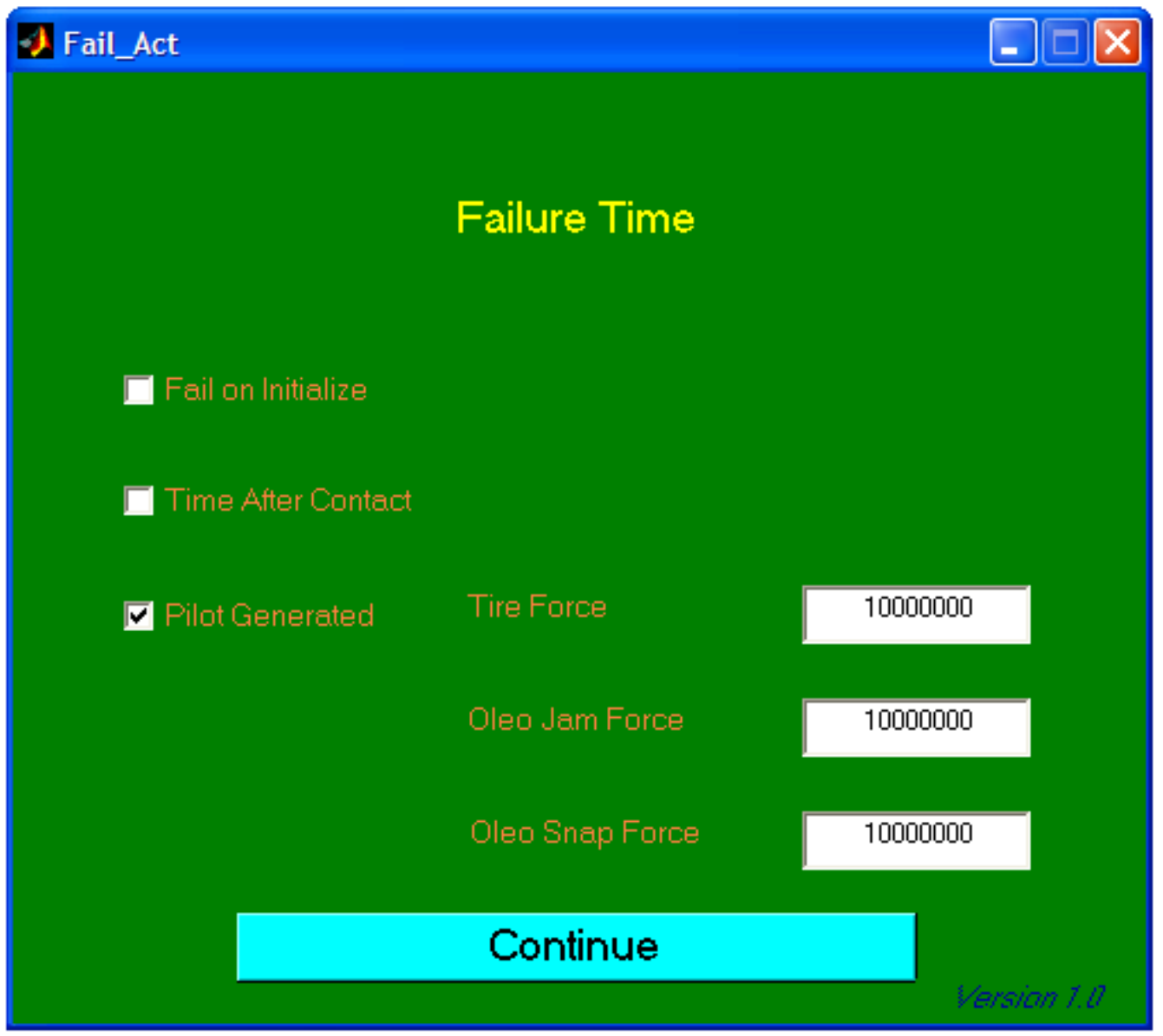

Figure 5. Selecting the Failure Trigger.

Once the failure triggers are selected, the starting location, or the flight condition, must be picked. The GUI that handles this is shown in Figure 6. As can be seen there are three options: short approach, on the runway or long approach. The short approach is useful for simulating the aircraft on landing operations. The long approach serves a similar mission but with more freedom. The runway is good for taxiing and take-off simulation. 


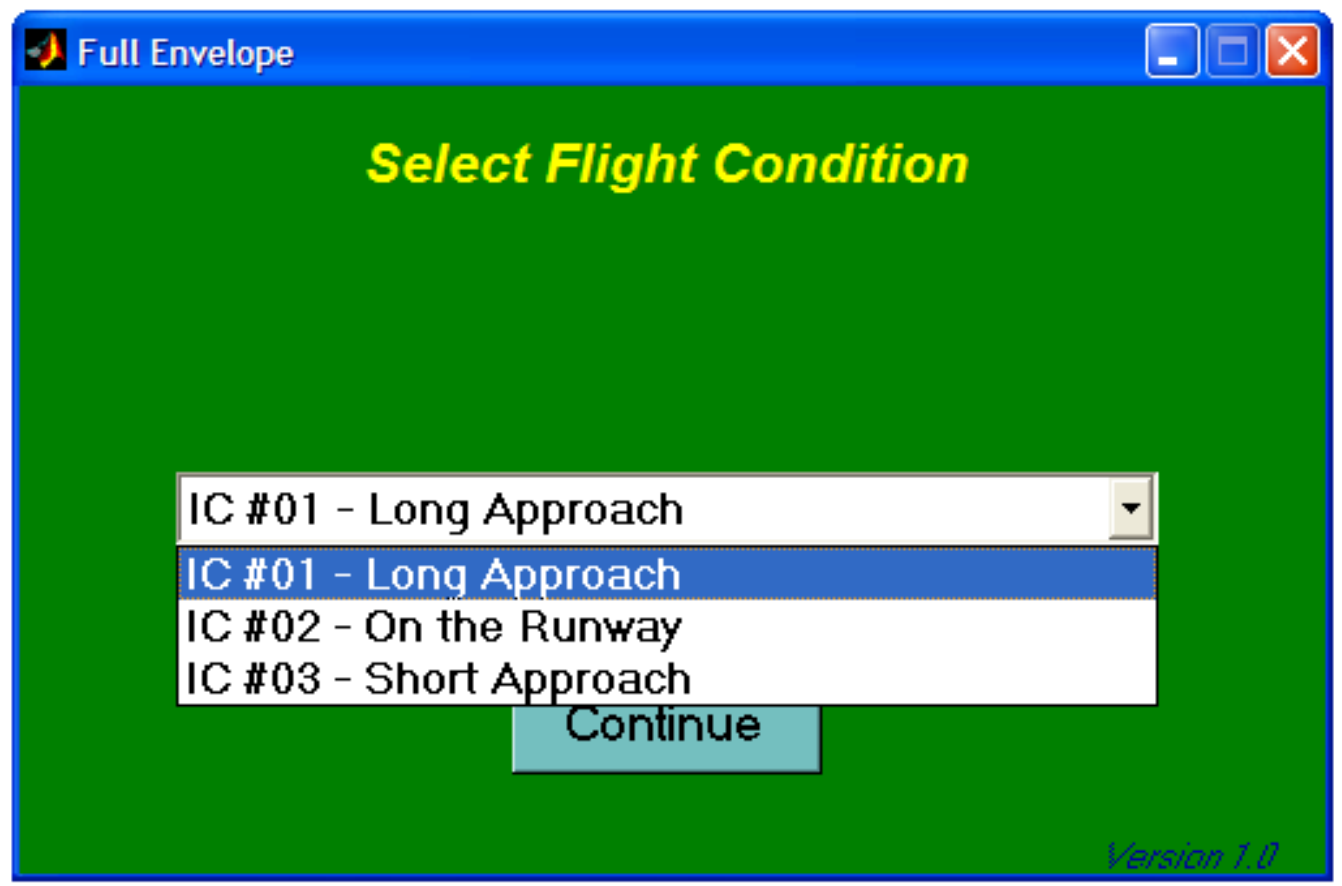

Figure 6. Selecting the Flight Condition.

With the flight condition chosen, simulation of the defined failure scenario can begin. Simulation is controlled with the module depicted in Figure 7. The first button is used to restart/stop the simulator. The next is used to choose a new predetermined failure scenario. The last button ends the landing failure simulation program. 


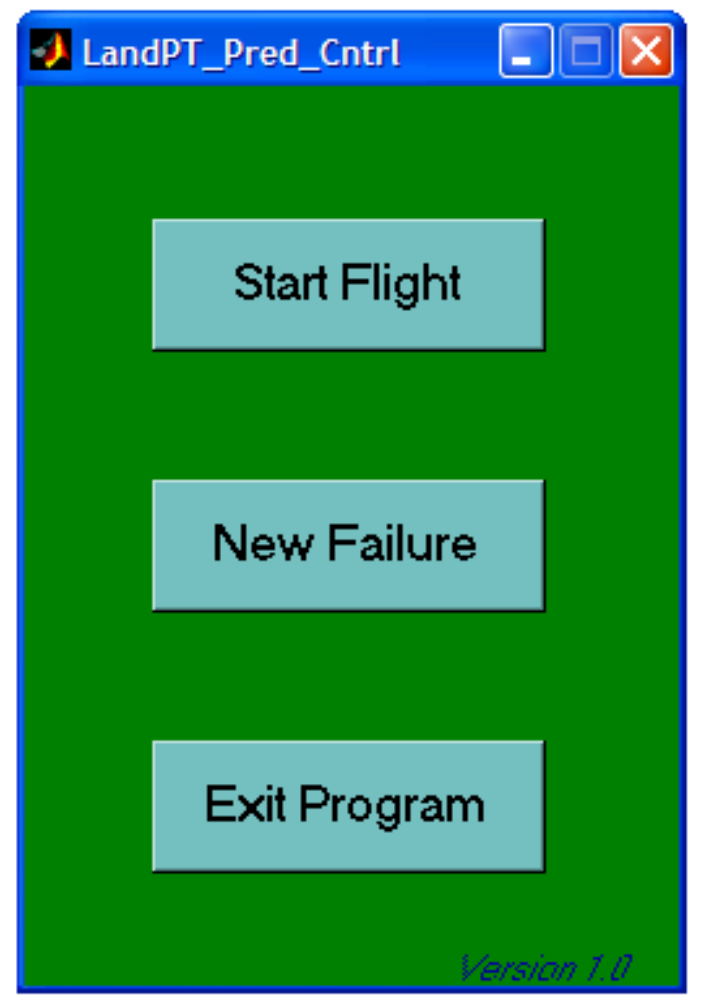

Figure 7. Predetermined Failure Control Module.

The Real Time failure injector works very similar to the predetermined one with a few minor differences. First, the flight condition is picked from the same menu. Since the failure will be triggered by the user at the desired time of the failure, there is no need to select it now. After this, the flight simulation can begin with a modified control module as depicted in Figure 8. This GUI unites the failure location selection menu with the control module. There is no New Failure option since restarting the model gives a new opportunity to simulate a new failure scenario. Loading the failure in the failure selection menu causes an immediate effect on the model. 


\section{Gear Position Failure Selection}

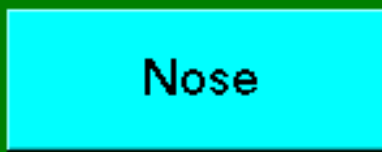

\section{Left Main}

Start Flight

\section{Right Main}

\section{Exit Program}

Figure 7. Predetermined Failure Control Module.

The Designer Usage option takes an entirely different approach to how the simulator is used. For this mode of operation to work, a virtual aircraft must be created by using the menu shown in Figure 8. Manually inserting each of the parameters is the most basic option in creating this virtual model. Additionally, there are Load/Save options to more quickly fill out the parameter list. Since the aircraft is a symmetric entity, there is also an option for the right main to mirror the left main. If another test had been ran prior to this simulation session, creation of the virtual aircraft model can be skipped and the results from the prior session can be viewed directly. Choosing this option or filling out the table and hitting continue brings up plot viewing menu. 


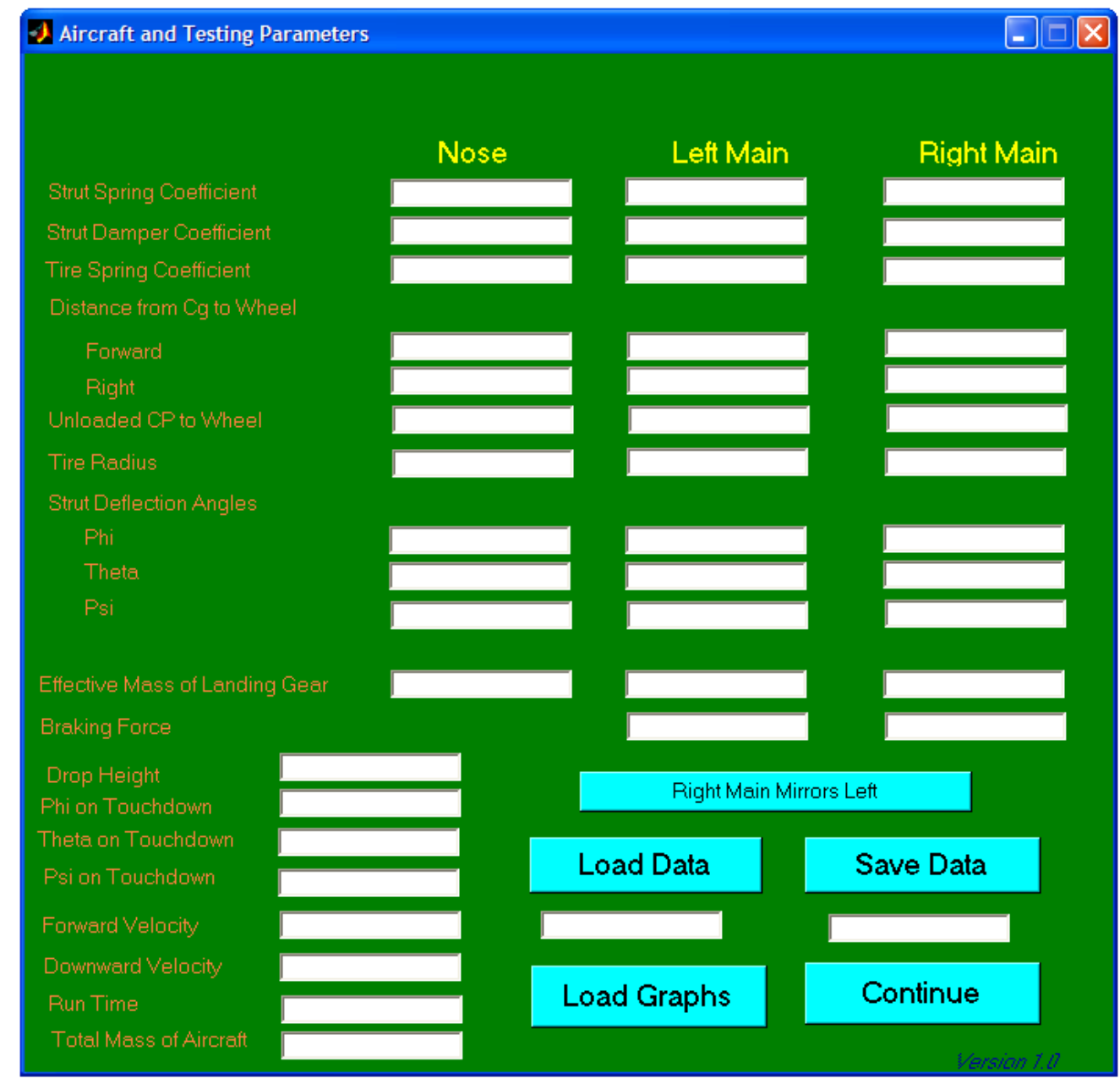

Figure 8. GUI used to Input Design Aide Parameters.

The plot viewing menu can be seen in Figure 9. From here, the states of the aircraft that the landing gear have an effect on can be displayed, namely the Euler angles and the velocities. Additionally, the position, velocity and acceleration of points corresponding to the wheel hub and the bottom of the wheel can also be viewed. To view one of the graphs, click the corresponding checkbox and click the Show Results pushbutton. Loading and saving the results can also be accomplished with this window. To round out the uses of the Designer Usage mode, the option to create a new aircraft model can be selected. The program ends by clicking the Exit Program button. 


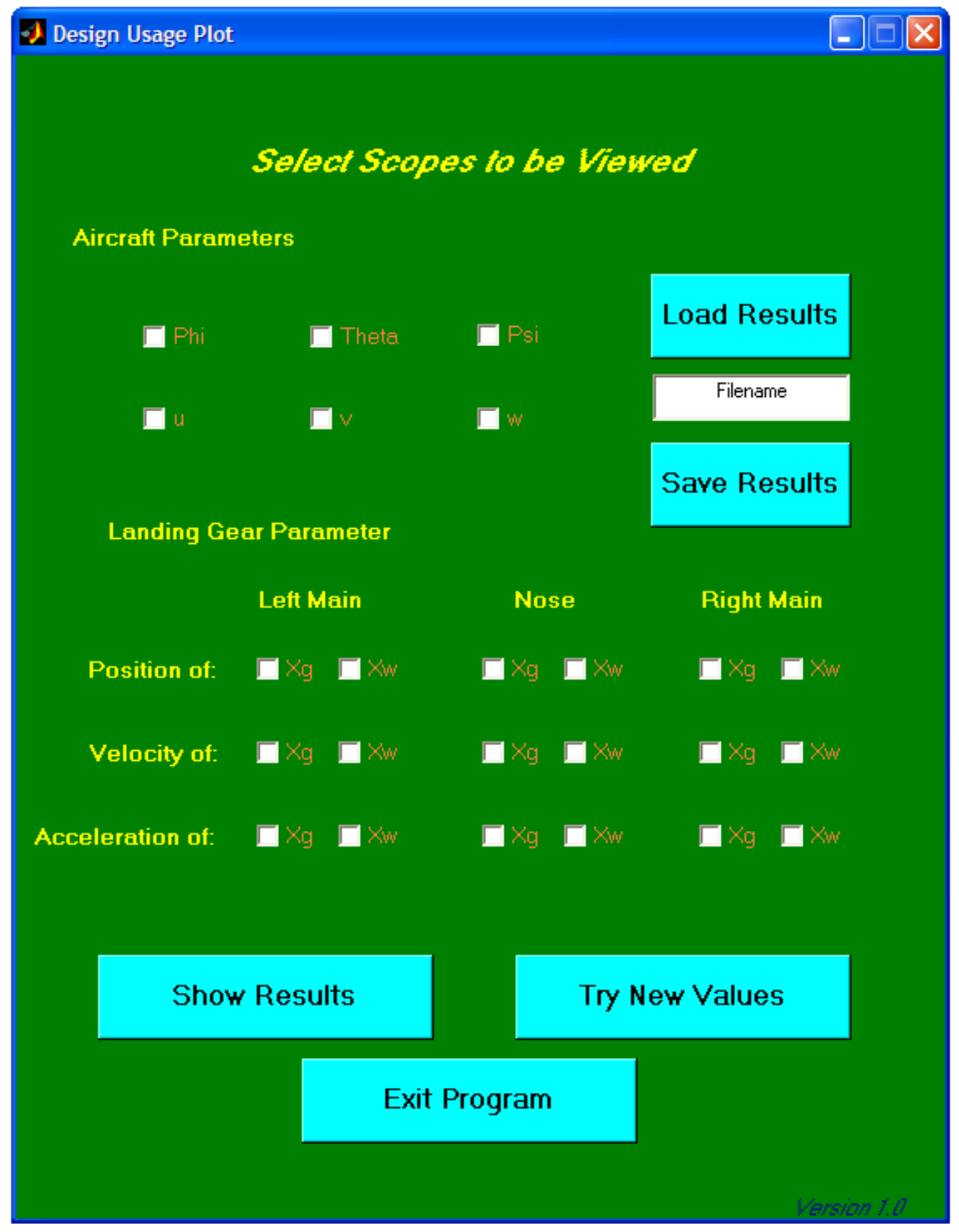

Figure 9. Menu used to Display Plots of Interesting Points

Within the landing system design mode, the aircraft was placed on the runway with the wheels just in contact with the surface of the runway with no vertical or horizontal velocity. In Figure 10, the original model derived from aircraft data was used. Figure 11 was generated with the spring value of the tires reduced by twenty percent. 


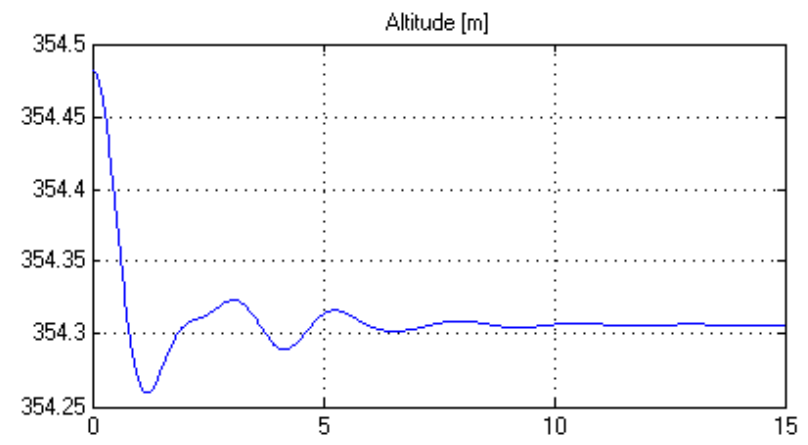

Figure 10. Normal Settling Response

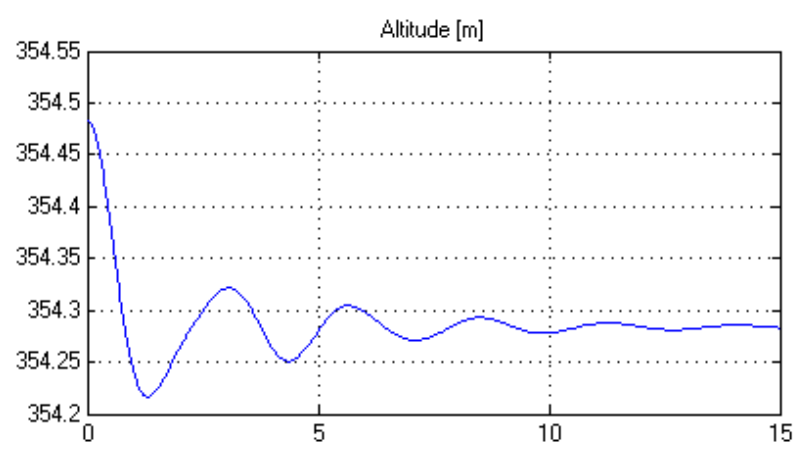

Figure 11. Response Reduced Tire Spring Constant 